\title{
Singular perturbation for the first eigenfunction and blow up analysis
}

\author{
David Holcman * $\quad$ Ivan Kupka ${ }^{\dagger}$
}

\begin{abstract}
On a compact Riemannian manifold $\left(V_{m}, g\right)$, we consider the second order positive operator $L_{\epsilon}=\epsilon \Delta_{g}+(b, \nabla)+c$, where $-\Delta_{g}$ is the Laplace-Beltrami operator and $b$ is a Morse-Smale (MS) field, $\epsilon$ a small parameter. We study the measures which are the limits of the normalized first eigenfunctions of $L_{\epsilon}$ as $\epsilon$ goes to the zero.

In the case of a general MS field $b$, such a limit measures is the sum of a linear combination of Dirac measures located at the singular point of $b$ and a linear combination of measures supported by the limit cycles of $b$.

When $b$ is a MS-gradient vector field, we use a Blow-up analysis to determine how the sequence concentrates on the critical point set. We prove that the set of critical points that a critical point belongs to the support of a limit measure only if the Topological Pressure defined by a variational problem (see [22]) is achieved there. Also if a sequence converges to a measure in such a way that every critical points is a limit point of global maxima of the eigenfunction, then we can compute the weight of a limit measure.This result provides a link between the limits of the first eigenvalues and the associated eigenfunctions. We give an interpretation of this result in term of the movement of a Brownian particle driven by a field and subjected to a potential well, in the small noise limit.
\end{abstract}

\section{Contents}

$\mathbf{1}$ Introduction $\quad 2$

1.1 Notations . . . . . . . . . . . . . . . . . . . 5

1.2 Normal coordinates . . . . . . . . . . . . . . . . . . 7

1.3 The self-adioint case . . . . . . . . . . . . . . . . . . 7

1.4 The first eigenvalue problem for the gradient case . . . . . . . . . . . . . . 13

1.5 The radial case : an example . . . . . . . . . . . . . . . . . . . . . 14

*Permanent address: Weizmann Institute of Science, department of Mathematic, Rehovot 76100, Israel. D.H is incumbent of the Madeleine Hass Russel Chair.

${ }^{\dagger}$ Université Paris VI, department of Mathematic, 175 rue du Chevaleret 75013 Paris, France. 
2 Blow up analvsis with no vector fields $\quad 15$

2.1 Main theorem . . . . . . . . . . . . . . . . . . . . . . . 16

2.2 Auxiliary propositions ... . . . . . . . . . . . . . . . . . . . 17

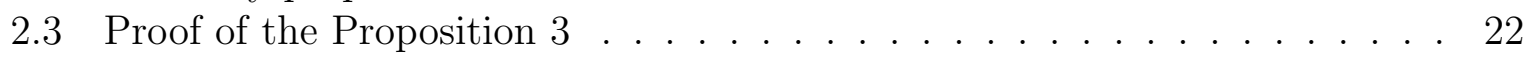

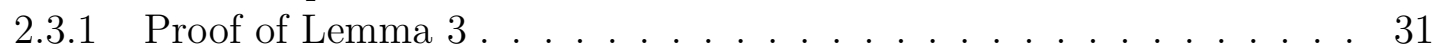

2.4 Proof of the main theorem . . . . . . . . . . . . . . . . . . . 32

2.5 The set of limit measures . . . . . . . . . . . . . . . . . . . . 37

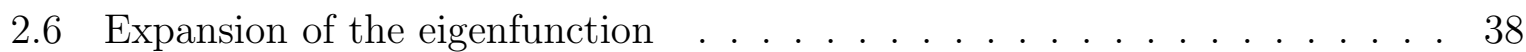

2.6 .1 Hermite functions . . . . . . . . . . . . . . . . . . . . . . . . . . 39

2.6.2 Expansion of the eigenvalue . . . . . . . . . . . . . . 40

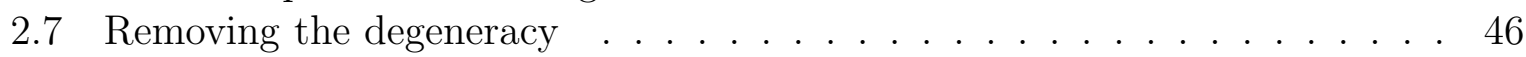

\begin{tabular}{|lll}
3 & Blow up analysis with a gradient vector field & 47
\end{tabular}

3.1 What do we learn from the Topological Pressure . . . . . . . . . . . . 47

3.2 blow-up Analvsis . . . . . . . . . . . . . . . . . . . . . . . . . . . . . 48

4 The general case for the first eigenfunction problem 54

4.0 .1 Proof of theorem $2 \ldots \ldots \ldots \ldots \ldots \ldots$

4.0.2 Remarks on the limit measures . . . . . . . . . . . . . . . . . 59

$4.1 \quad$ Final Remark . . . . . . . . . . . . . . . . . . . . . . . . . . . . . . . . 59

$\begin{array}{lll}5 \text { Appendix 1 } & 59\end{array}$

5.1 Local construction of Lvapunov functions $\ldots \ldots \ldots$

5.2 Global construction of Lvapunov functions . . . . . . . . . . . . . . . . 62

5.2 .1 Local Lvapunov functions . . . . . . . . . . . . . . . . . . 63

5.2 .2 Construction of the global Lvapunov function . . . . . . . . . . . 63

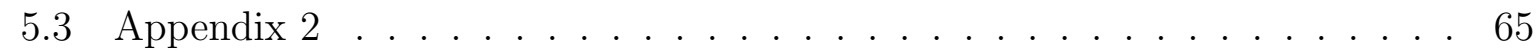

\section{Introduction}

Let $\left(V_{m}, g\right)$ denote a compact Riemannian manifold of dimension $m \geq 2$, with no boundary. $-\Delta_{g}$ denotes the associated Laplace-Beltrami operator . In this paper we consider a second order elliptic operator depending on the parameter $\varepsilon>0$,

$$
L_{\epsilon}=\epsilon \Delta_{g}+\theta(b)+c,
$$

where $b$ denotes a $C^{\infty}$ vector field on $V, c$ a strictly positive $C^{\infty}$ function on $V_{m}$ and $\theta(b)$ the Lie derivative operator associated to $b: \theta(b) u=d u(b)$ for any function $u$ on $V$.

$L_{\epsilon}$ is a positive operator to which the Krein-Rutman [25] theorem can be applied. Hence the smallest eigenvalue $\lambda_{\epsilon}$ of $L_{\epsilon}$ is simple and strictly positive. The associated eigenspace is generated by a strictly positive function $u_{\epsilon}$, normalized in $L_{2}\left(V_{m}\right)$. The behavior of $\lambda_{\epsilon}$ as $\epsilon$ goes to zero has been extensively studied [20, 21, 22, 3, 4,

In the case when the $\omega$-set is a disjoint union of compact invariant hyperbolic set, under a mild additional assumption Y. Kifer has proved that the limit of $\lambda_{\epsilon}$ as $\epsilon$ goes to 
zero is the topological pressure (TP), associated to the flow of $b$ and the potential $c$ (for definitions and details see [22]).

Unfortunately, much less is known about the weak limits of the eigenfunctions $u_{\epsilon}$, as $\epsilon$ goes to zero. By weak limits, we mean the limits of the measures $u_{\epsilon}^{2} d v o l_{g}$ in the weak topology of measures. Past results include the case when $V_{m}$ is a bounded domain of $\mathbb{R}^{n}$, the limit sets of the vector field $b$ is reduced to a single point, $\lambda_{\epsilon}$ and $u_{\epsilon}$ are related to the zero Dirichlet boundary condition.

When this singular point is attractive, $u_{\epsilon}$ as $\epsilon$ goes to zero, converges to a Dirac measure supported by the attractor. When it is repulsive $u_{\epsilon}$ converges to a constant on every compact sub-domain of the domain of definition (see [2, 3]). When the attractive set consists of a single limit cycle, the first eigenvalue converges to the average of the potential $c$ of the PDE, along the cycle (see [16] ), $\lim _{\epsilon \rightarrow 0} \lambda_{\epsilon}=\frac{\int_{0}^{T} c\left(x_{0}(t)\right) d t}{T}$, where $x_{0}$ parametrizes the limit cycle of period $\mathrm{T}$, the proof uses a stochastic approach. It is interesting to note that this approach and the deterministic methods give complementary results.

When $b=0$, the equation (11) reduces to:

$$
\epsilon \Delta_{g} u_{\varepsilon}+c u_{\varepsilon}=\lambda_{\varepsilon} u_{\varepsilon}
$$

The limits of the $u_{\epsilon}^{2} d v o l_{g}$ (normalized by: $\int_{V} u_{\varepsilon}^{2} d v o l_{g}=1$ ) has been studied for example in (31, 30, 11]) and is known as the semi-classical limit. In particular when the potential $c$ has a double well in $P_{1}$ and $P_{2}$ ( that is an absolute non-degenerate minimum at each points), it is well known that the limits of the measures $u_{\epsilon}^{2} d v o l_{g}$ as $\varepsilon$ goes to 0 can concentrate on those points only and in the distribution sense, as $\epsilon$ goes to 0

$$
u_{\epsilon}^{2} d v o l_{g} \rightarrow c_{1} \delta_{P_{1}}+c_{2} \delta_{P_{2}}
$$

where $c_{1}+c_{2}=1$. As far as we know, nobody has addressed the question of computing explicitly the coefficients $c_{1}$ and $c_{2}$ in general. Of course, under additional assumptions, for example, when the $u_{\epsilon}$ are invariant by a group of isometries, $c_{1}=c_{2}=\frac{1}{2}$. It is not clear at present time whether or not those coefficients are unique. We will see here that those coefficients depend only on the Hessian of the potential at the points $P_{1}, P_{2}$ and on the limit of the ratio (called the modulating ratio) of the local maximum to the global maximum of the $u_{\epsilon}$, but they do not depend on anything else.

Sometimes, as explained in 31, one of the coefficient is zero and the limits of the measures $u_{\epsilon}^{2} d v o l_{g}$ concentrate on the remaining point. In that case, we use the terminology of 31 and say that the degeneracy is removed. The degeneracy can be removed by looking at the expansion of $\lambda_{\epsilon}$ in terms of $\epsilon$ in the following way. $\lambda_{\epsilon}$ depends on the values of the potential and of the derivatives of the metric tensor at the minimum points. The Taylor expansions of $\lambda_{\epsilon}$ have to be the same to each order at $P_{1}$ and $P_{2}$. If this is not the case then only one point will be charged. In this paper we compute the necessary conditions up to order 4, in order that the degeneracy can be removed in terms of the geometry. These conditions narrow down the set of points which can be charged ( i.e.the set of points where the coefficient $c_{k}$ is strictly positive). 
We conjecture that the coefficients $c_{k}$ are unique and when the degeneracy cannot be removed they are charged by a global maximum sequence (the modulating ratio is equal to 1$)$.

When $b$ is a gradient $b=\nabla \phi$, the limits of the measures $u_{\epsilon}^{2} d v o l_{g}$ as $\varepsilon$ goes to 0 can concentrate on the critical points of $b$ only. The concentration results are proved for the normalized measures

$$
\frac{e^{-\frac{\phi}{\epsilon}} u_{\epsilon}^{2} d v o l_{g}}{\int_{V_{m}} e^{-\frac{\phi}{\epsilon}} u_{\epsilon}^{2} d v o l_{g}}
$$

and we shall see how the potential $c$ interacts with the field $b$ to select the points that will be charged. This set is the set of points where the Topological Pressure attains its minimum (see [22]).

When $b$ is a not necessarily the gradient of function, very few results are known about the behavior of the measure $u_{\epsilon}^{2} d v o l_{g}$ as $\epsilon$ goes to zero, because it not clear by which function if any, the function $\phi$ should be replaced in expression 2, Recall that the operator $L_{\epsilon}$ with general drifts have not been considered before, especially in the context of Quantum Mechanics, because it has an interpretation only in the context of diffusion. Actually, on the striking results in this paper is that $\phi$ can be replaced by any global Lyapunov function $L$ associated to the field $b$. Using a weight which is a Gaussian in the Lyapunov function, is a crucial input into the problem because it enables us to "filter" the limits in order to get results about the concentration. Using a Lyapunov type function, as presented in section 4, is by far more general than using a solution of the Hamilton-Jacobi equation,

$$
|\nabla L|^{2}+(b, \nabla L)=0
$$

as it is currently used in the formal expansion of the WKB theory. Another striking result is that the possible limit measures are supported by specific limit cycles of the field, which are not necessarily the attractors. On a compact Riemannian manifold, the existence of a solution to the Hamilton-Jacobi equation is not always guarrante to be smooth, while in appendix 2, we prove the existence of a smooth Lyapunov function. In this context, we prove that the measure

$$
\frac{e^{-\frac{L}{\epsilon}} u_{\epsilon}^{2} d v o l_{g}}{\int_{V_{m}} e^{-\frac{L}{\epsilon}} u_{\epsilon}^{2} d v o l_{g}}
$$

can concentrate on the limit cycle of a Morse-Smale dynamical system, without any additional assumptions. Finally, we give explicit results about the decay of the eigenfunction sequence near the concentration set and analyze the influence of the Riemannian geometry.

\section{Our main results in this paper are:}

- In theorem [7 of section 4, we prove that the limits of the normalized eigenfunctions $u_{\epsilon}$ as $\epsilon$ tends to zero, are measures concentrated on the limit sets of a Morse-Smale field $b$. The possible limit measures are supported by specific limit cycles of the field, which are not necessarily the attractors. 
- In theorem 2 we prove that the blown up function is the standard solution of the Harmonic Oscillator. This lead us to theorem 3 to give a precise expression of the coefficient of the limit measures and to characterize the set of minimum of $c$ which are in the support of the limit measures. As a byproduct of the analysis, we obtain some estimates of the velocity at which the sequence of local maximum $P_{\epsilon}$ converges to a critical point of the potential.

- In theorem4, we give a geometric interpretation of the coefficients of the expansion of $\lambda_{\epsilon}$ in terms of the power of $\sqrt{\epsilon}$. These coefficients are invariant of the couple (Riemannian metric, potential). The value of the second term of the expansion of $\lambda_{\varepsilon}$ in powers of $\sqrt{\varepsilon}$ is computed, using minimax procedures, but this value has to be compared to the one obtained in [12], based on the WKB formula. The result obtained here are based on the variational approach and seems to lead to results that have to be compare with the results obtained by the formal WKB expansion of the first eigenfunction.

- In theorem 6, we study the case where the field b is a gradient of a Morse-Smale function. We prove that the concentration of the first eigenfunction occurs at the critical points of $b$ where the Topological Pressure is attained. In addition, the weights of the limit measures is given under specific assumptions.

\section{Remarks.}

Some of the results presented here complete and extend also some previous work in analysis [17, 18, 2, 3, 4,. In addition, at the time the results of this paper were announced [13, we were not aware of any reports about the fine selection by the limit of the eigenfunction sequence of a subset of critical points of the potential. The detail of this selection process is presented in the subsection 2.6.2 and 2.7 and should clarify how the metric and the potential are involved. This results extend in particular the work of [30, 32, 31].

\section{$1.1 \quad$ Notations}

$\left(x^{1}, \ldots, x^{m}\right): U \longrightarrow \mathbb{R}$, is a coordinate patch on $V$ and $f$ a function on $V$ : 
$d_{g}: V \times V \longrightarrow>\mathbb{R}_{+}:=$distance associated to $g$

$(,)_{g}:=$ scalar product associated to $\mathrm{g}$

$\exp _{x}: T_{x} V \longmapsto V:=\operatorname{exponential~map~of~} g$ with pole $x$

$\operatorname{vol}_{g}:=$ volume measure associated to $g$

$L^{2}(E):=L^{2}$ space associated to $\operatorname{vol}_{g}$ on the subset $E$ of $V$

$L^{2}:=L^{2}(V)$

$\Delta_{g}:=$ negative Laplacian associated to the metric

$b:=$ vector field on $V$

$\theta(b):=$ Lie derivation operator associated to $b$

$\nabla:=$ gradient associated to $g$

$M(V):=$ space of all probability measures on $V$

$C^{\infty}$ topology:=uniform convergence of all the derivatives on compact sets

$g:=\sum_{i j=1}^{m} g_{i j} d x_{i} d x_{j}$

$\Delta_{g}:=-\frac{1}{\sqrt{\operatorname{det}(g)}} \sum_{i j=1}^{m} \frac{\partial}{\partial x_{i}} \sqrt{\operatorname{det}(g)} g^{i j} \frac{\partial}{\partial x_{j}}$

$g^{i j}:=$ inverse matrix of $g_{i j}$

$\operatorname{det}(g):=\operatorname{det}\left(g_{i j}\right)$

$\Gamma_{i j}^{k}:=$ Christoffel symbols of $g_{i j}$

$\Gamma_{i j}^{k}=\frac{1}{2} g^{k l}\left(\frac{\partial g_{i l}}{\partial x_{j}}+\frac{\partial g_{j l}}{\partial x_{i}}-\frac{\partial g_{i j}}{\partial x_{l}}\right)$

$R_{i j k}^{\cdots l}:=\frac{\partial \Gamma_{j k}^{l}}{\partial x_{i}}-\frac{\partial \Gamma_{i k}^{l}}{\partial x_{j}}+\sum_{n=1}^{m}\left[\Gamma_{i n}^{l} \Gamma_{j k}^{n}-\Gamma_{j n}^{l} \Gamma_{i k}^{n}\right]$

$R_{i j k l}:=\sum_{n=1}^{m} g_{\ln } R_{i j k}^{\cdots \cdot n}$

$R i c_{k l}:=\sum_{j=1}^{m} R_{j k l j}$

$R:=\sum_{j=1}^{m} R i c_{j j}=\sum_{i, j=1}^{m} R_{i j j i}$

$\theta(b):=\sum_{i=1}^{m} b^{i} \frac{\partial}{\partial x^{i}}$

$d x^{i}(\nabla f):=\sum_{i=1}^{m} g^{i j} \frac{\partial f}{\partial x^{j}}, 1 \leq i \leq m$ 


\subsection{Normal coordinates}

For each $\mathrm{P} \in \mathrm{C}_{\min }$, we choose a normal coordinate system $\left(x_{1}, \ldots x_{m}\right): U_{P} \longrightarrow \mathbb{R}$, centered at $\mathrm{P}$, defined on a domain $\mathrm{U}_{P}$ such that:

1. $\mathrm{x}_{1} \times \ldots \times \mathrm{x}_{m}\left(U_{P}\right)$ contains the closed ball $\mathrm{B}_{P}(\delta)$ centered at $\mathrm{P}$ and having radius $\delta>0$.

2. for all $\mathrm{i}, \mathrm{j}, 1 \leq \mathrm{i}, \mathrm{j} \leq \mathrm{m}, \frac{\partial^{2} c}{\partial x_{i} \partial x_{j}}(P)=\lambda_{i}(P) \delta_{i j}$.

3. $\mathrm{U}_{P} \cap \mathrm{U}_{Q}=\varnothing$ for all $\mathrm{P}, \mathrm{Q} \in C_{\min }, \mathrm{P} \neq \mathrm{Q}$.

In the following we will identifie $\mathrm{U}_{P}$ with the open neighborhood of $\mathrm{O}$ in $\mathbb{R}^{m}, x_{1} \times \ldots \times x_{m}\left(U_{P}\right)$. For $\mathrm{r}$ such that $\mathrm{B}_{P}(\mathrm{r}) \subset \mathrm{U}_{P}, \mathrm{~B}_{P}(\mathrm{r})$ will denote both the geodesic ball centered at $\mathrm{P}$ and of radius $\mathrm{r}$ in $\mathrm{V}$ or its image by the mapping $x_{1} \times \ldots \times x_{m}$. For the sake of streamlining the notations, we shall commit the abuse, when working with the coordinate system $\left(x_{1}, \ldots x_{m}\right): U_{P} \longrightarrow \mathbb{R}$, of denoting by $B_{P}(\mathrm{r})$ the ball of center $\mathrm{O}$ and radius $\mathrm{r}$ whatever the value of $\mathrm{r}(\geq 0$ of course $)$.

Now a few words about the blow-up procedure. On a manifold $V$ let $P$ be a point and a chart $\left(U, x_{1}, \ldots x_{m}\right)$ of $\mathrm{V}$ centered at $\mathrm{P}: x_{i}(\mathrm{P})=0,1 \leq \mathrm{i} \leq m$. The blow-up of power $\mathrm{t}>0$ associated to $P$ and the chart $\left(U, x_{1}, \ldots x_{m}\right)$ is the diffeomorphism $B l_{t}: U \rightarrow \mathbb{R}^{m}$, $B l_{t}(Q)=\left(\frac{1}{t} \mathrm{x}_{1}(Q), \ldots, \frac{1}{t} x_{m}(Q)\right)$. All functions, tensors, differential operators can be transported to the open subset $B l_{t}(\mathrm{U})$. Suitably normalized by a power of t, they will have limits when $t$ goes to 0 which will be defined on $\mathbb{R}^{m}$. These limits contain a trove of information about the behaviour of the original objects in the neighborhood (more precisely in the infinitesimal neighborhood) of $P$. To simplify the notations we shall write: $\frac{1}{t} Q$ instead of $B l_{t}(Q), \frac{1}{t} A$ instead of $B l_{t}(A)$ if $A$ is a subset of $U$ and so on.

Recall that a field $b$ is Morse-Smale MS if : (i)the recurrent set of $b$ consists of a finite number of hyperbolic points and periodic orbits (ii) each pair of stable or unstable manifolds of these points or orbits intersect transversally. If moreover $b$ is a gradient of a function with respect to the metric $g, b$ will be called a MS gradient field.

\subsection{The self-adjoint case}

In the self adjoint case the vector field $b$ is zero. This assumption simplifies the problem because it can be handled by variational methods. Theorem 1 below seems well-known but we could not find a proof for Riemannian manifolds, using deterministic techniques in the literature. Hence as a starting point, we provide a simple one here. 


\section{Notations.}

For each $\mathrm{P} \in \mathrm{C}_{\text {min }}$, we choose a normal coordinate system $\left(x_{1}, \ldots x_{m}\right): U_{P} \longrightarrow \mathbb{R}$, centered at $\mathrm{P}$, defined on a domain $U_{P}$ such that:

1. $x_{1} \times \ldots \times x_{m}\left(U_{P}\right)$ contains the closed ball $\mathrm{B}_{P}(\delta)$ centered at $\mathrm{P}$ and having radius $\delta>0$.

2. for all $\mathrm{i}, \mathrm{j}, 1 \leq \mathrm{i}, \mathrm{j} \leq \mathrm{m}, \frac{\partial^{2} c}{\partial x_{i} \partial x_{j}}(P)=\lambda_{i}(P) \delta_{i j}$.

3. $U_{P} \cap U_{Q}=\varnothing$ for all $P, Q \in C_{\min }, P \neq Q$.

In the following we will identifie $U_{P}$ with the open neighborhood of $\mathrm{O}$ in $\mathbb{R}^{m}, x_{1} \times \ldots \times x_{m}\left(U_{P}\right)$. For $\mathrm{r}$ such that $B_{P}(\mathrm{r}) \subset U_{P}, B_{P}(\mathrm{r})$ will denote both the geodesic ball centered at $\mathrm{P}$ and of radius $\mathrm{r}$ in $\mathrm{V}$ or its image by the mapping $x_{1} \times \ldots \times x_{m}$. For the sake of streamlining the notations, we shall commit the abuse while working with the coordinate system $\left(\mathrm{x}_{1}\right.$, $\left.\ldots \mathrm{x}_{m}\right): U_{P} \longrightarrow \mathbb{R}$, of denoting by $B_{P}(\mathrm{r})$ the ball of center $\mathrm{O}$ and radius $\mathrm{r}$ whatever the value of $r(\geq 0$ of course $)$.

Theorem 1 Consider the first eigenvalue problem for the operator $\Delta_{g}+c$ where $c$ is a function with a finite set of minimum points, $C_{\min }$, which are not degenerate (in the sense of Morse). Assume that the first eigenvalue of the operator $\lambda_{\varepsilon}$ is positive. $\lambda_{\varepsilon}$ has the following variational expression:

$$
\lambda_{\epsilon}=\inf _{u \in H^{1}(V)-\{0\}} \frac{\int_{V}\left[\epsilon|| \nabla u \|_{g}^{2}+c u^{2}\right] d v o l_{g}}{\int_{V} u^{2} d v o l_{g}}
$$

Then, when $\epsilon$ converges to zero, $\lambda_{\epsilon}$ converges to the minimum of the function $c$ and the set of weak limits, when $\epsilon$ goes to zero, of the family of measures $\frac{u_{\epsilon}^{2} d v o l_{g}}{\int_{V} u_{\epsilon}^{2} d v o l_{g}}$ defined by the positive solutions $u_{\epsilon}$ of the PDE,

$$
\epsilon \Delta_{g} u_{\epsilon}+c u_{\epsilon}=\lambda_{\epsilon} u_{\epsilon} \text { on } V
$$

is contained in the simplex

$$
M=\left\{\nu=\sum\left(\gamma_{P} \delta_{P} \mid P \in C_{\min }\right) \| \sum \gamma_{P}=1, \gamma_{P} \geq 0\right\}
$$

of all probability measures with support in the finite set $C_{\min }$ where $\delta_{P}$ denotes the Dirac measure at the point $P$.

Remarks. $u_{\epsilon}$ is uniquely defined up to a multiplicative constant by the Krein-Rutman theorem (see [23]).

In the following proofs, over and over, we will chose appropriate sub-sequences of $\left\{u_{\epsilon}\right.$, $\epsilon>0\},\left\{\lambda_{\varepsilon} \mid \varepsilon>0\right\}$ and so on, without saying so explicitly: In order to keep the notations simple we will write $u_{\epsilon}, \lambda_{\varepsilon}, \ldots$ instead of a sequence $\left(u_{\epsilon_{k}} \mid k=1 ..\right),\left(\lambda_{\epsilon_{k}} \mid k=1 ..\right)$.. 
Proof: without restricting the generality we can assume that $c \geq 0$. To start note that $\lambda_{\epsilon} \geq \min _{V} c \geq 0$. Using a constant as test function in the functional that define the first eigenvalue, we get:

$$
\lambda_{\epsilon} \leq \frac{\int_{V} c d v o l_{g}}{v o l_{g}(V)} \leq \sup _{V} c
$$

$\lambda_{\epsilon}$ is a decreasing function of $\epsilon$ because the functional decreases with $\epsilon . u_{\epsilon}$ being bounded, there exists a sub-sequence which converges weakly and

$$
\forall \phi \in H^{1}(V), \int_{V}\left[\epsilon(\Delta \phi) u_{\epsilon}+c \phi u_{\epsilon}\right]=\lambda_{\epsilon} \int_{V} u_{\epsilon} \phi
$$

To obtain an upper estimate for $\lambda_{\epsilon}$, consider the following radial function defined on $V_{m}$ for $m>2$ :

$$
\begin{array}{r}
\phi_{\mu}(r)=\frac{\mu^{(m-2) / 2}}{\left(r^{2}+\mu^{2}\right)^{(m-2) / 2}}-\frac{\mu^{(m-2) / 2}}{\left(\delta^{2}+\mu^{2}\right)^{(m-2) / 2}}, \text { on } B_{P}(\delta) \\
=0, \text { on } V-B_{P}(\delta)
\end{array}
$$

Then $\phi$ belongs to $H^{1}(V)$. We use this function in the energy function $I(u)=\frac{\epsilon \int_{V}\left(\|\nabla u\|_{g}^{2}+c u^{2}\right) d v o l_{g}}{\int_{V} u^{2} d v o l_{g}}$. The following standard computation gives an estimate of $I\left(\phi_{\mu}\right)$ :

$$
\epsilon \int_{V}\left\|\nabla \phi_{\mu}\right\|_{g}^{2} d v o l_{g}=\epsilon\left(\omega_{m-1} \int_{B_{\delta / \mu}(P)} r^{m-1} d r \frac{1}{\left(1+r^{2}\right)^{m}}+o(\mu)\right)=\epsilon c(m)+o(\mu) \epsilon
$$

where the constant $c(m)$ depends only on the dimension $\mathrm{n}$ and $\omega_{m-1}$ is the volume of the unit (n-1)-sphere of $\mathbb{R}^{m}, r=d(P, Q)$ is the geodesic distance and $\delta$ is less than the injectivity radius. We have to evaluate the other quantities in the functional. Using the change of variable $x=y \mu$, we get:

$$
\int_{V} c \phi_{\mu}^{2} d v o l_{g}=\int_{B_{\delta / \mu}(P)} a \phi_{\mu}^{2} d v o l_{g}=\mu^{2} V(P) \omega_{m-1} J(\delta / \mu)+o(\mu),
$$

where

$$
J(\delta / \mu)=\int_{B_{\delta / \mu}(P)}\left(\frac{1}{\left(r^{2}+1\right)^{(m-2) / 2}}-\frac{1}{\left(\delta^{2}+1\right)^{(m-2) / 2}}\right)^{2} d v o l_{g}
$$

We have $J(\delta / \mu)=O(1)$ for $n \geq 5$ and for $\mathrm{n}=4, J(\delta / \mu)=O(\ln \delta / \mu)$

$$
\int_{B_{\delta / \mu}(P)} c \phi_{\mu}^{2} d v o l_{g}=\int_{B_{\delta / \mu}(P)} c \mu^{2}\left(\frac{1}{\left(r^{2}+1\right)^{(m-2) / 2}}-\frac{1}{\left(\delta^{2}+1\right)^{(m-2) / 2}}\right)^{2} d v o l_{g}
$$

then after some computations:

$$
\int_{V} \phi_{\mu}^{2} d v o l_{g}=\mu^{2} \omega_{m-1} J(\delta / \mu)+o(\mu)
$$


Taking $\mu=\epsilon^{1 / 3}$, we get the expansion

$$
I\left(\phi_{\epsilon}\right)=c(P)+o(1)
$$

where $\mathrm{P}$ is a minimal point of $c$.

In the 2-dimensional case, we can consider the test function

$$
\begin{aligned}
\phi_{\mu}(r)=\frac{\mu^{p}}{\left(r^{2}+\mu^{2}\right)^{p}}-\frac{\mu^{p}}{\left(\delta^{2}+\mu^{2}\right)^{p}}, & \text { on } B_{P}(\delta) \\
& =0, \text { on } V-B_{P}(\delta)
\end{aligned}
$$

where $p$ satisfies $0<p \leq 1 / 2$. The same computations as before, taking $\mu^{4}=\epsilon$, gives the result. Finally

$$
\begin{aligned}
\min _{V} c & \leq \lambda_{\epsilon} \leq c(P)+o(1) \\
\lim _{\epsilon \rightarrow+\infty} \lambda_{\epsilon} & =\min _{V} c
\end{aligned}
$$

Using the energy equation, we have $\lambda_{\epsilon} \geq \epsilon \int_{V} \mid\left[\mid \nabla u_{\epsilon} \|_{g}^{2}+\min _{V} c\right]$, which forces $\epsilon \int_{V}\left\|\nabla u_{\epsilon}\right\|_{g}^{2}$ $d v o l_{g}$ to tend to zero as $\epsilon$ goes to zero:

$$
\lim _{\varepsilon->0} \epsilon \int_{V}\left\|\nabla u_{\epsilon}\right\|_{g}^{2} d v o l_{g}=0
$$

Also for any $\phi \in \mathrm{C}^{2}(\mathrm{~V})$ multiplying equation (5) by the function $\phi u_{\epsilon}$ and integrating by part gives :

$$
\int_{V}\left[\epsilon\left\|\nabla u_{\epsilon}\right\|_{g}^{2} \phi+c u_{\epsilon}^{2} \phi+\epsilon(\Delta \phi) \frac{u_{\epsilon}^{2}}{2}\right] d v o l_{g}=\lambda_{\epsilon} \int_{V} \phi u_{\epsilon}^{2} d v o l_{g}
$$

where $\int_{V} u_{\epsilon}^{2}=1$. Because the first term and the last term on the left hand-side are converging to zero (see 8), we obtain that

$$
\lim _{\epsilon \rightarrow 0} \int_{V} \phi u_{\epsilon}^{2}\left(c-\lambda_{\epsilon}\right) d v o l_{g}=0
$$

But:

$$
\begin{gathered}
0 \leq \int_{V}\left(c-\min _{V} c\right) \phi u_{\varepsilon}^{2} d v o l_{g} \leq \int_{V}\left(c-\lambda_{\epsilon}\right) \phi u_{\epsilon}^{2} d v o l_{g}=0 \\
\lim _{\varepsilon \rightarrow 0} \int_{V}\left(c-\min _{V} c\right) \phi u_{\varepsilon}^{2}=0
\end{gathered}
$$

Moreover if $u$ denotes a weak limit of $u_{\epsilon}$ as $\epsilon$ goes to zero, relation (10) implies that $\forall \phi \in C^{1}(V)$ :

$$
\int_{V}\left(c-\min _{V} c\right) \phi u^{2} d v o l_{g}=0
$$


Hence if the set $\left\{P \in V \mid c(P)=\min _{V} c\right\}$ is of measure 0 , we conclude that $u=0$. Hence all weak limits of $u_{\epsilon}$ are zero and the sequence $u_{\epsilon}$ concentrates, as we shall see shortly.

Note that relation (10) implies that for any $\psi \in C(V)$, which is zero in a neighborhood of $\mathrm{C}_{\min }$ :

$$
\lim _{\varepsilon->0} \int_{V} \psi u_{\varepsilon}^{2} d v o l_{g}=0
$$

If $\mathrm{A}$ is a measurable subset of $\mathrm{V}$ such that $\bar{A} \cap C_{\min }=\varnothing, \bar{A}$ denoting the closure of $\mathrm{A}$, applying (12) to a positive continuous function $\psi$ with support disjoint from $\mathrm{C}_{\min }$ and $\psi \geq 1$ on $\mathrm{A}$ we get:

$$
\lim _{\varepsilon->0} \int_{A} u_{\varepsilon}^{2} d v o l_{g}=0
$$

In fact, we can prove that any sequence $\left\{\varepsilon_{n} \mid n \in \mathbb{N}\right\}$ converging to 0 , contains a subsequence $\left\{\varepsilon_{n_{k}} \mid k \in \mathbb{N}\right\}$ such that the corresponding $u_{\epsilon_{n_{k}}}$ converges to a convex sum of Dirac distributions located at the minimum points of $c$. We assume the $u_{\varepsilon_{n}}$ normalized so that $\int_{V} u_{\epsilon_{n}}^{2}=1$. Consider the following decomposition

$$
\int_{V} \phi u_{\epsilon}^{2} d v o l_{g}=\sum_{P \in C_{\min }} \int_{B_{P}(\delta)}((\phi-\phi(P))+\phi(P)) u_{\epsilon}^{2} d v o l_{g}+\int_{V-\cup_{P \in C_{\min }} B_{P}(\delta)} \phi u_{\epsilon}^{2} d v o l_{g}
$$

Relation (13)implies that:

$$
\int_{V} \phi u_{\epsilon}^{2} d v o l_{g}-\phi(P) \sum_{P \in C_{\min }} \int_{B_{P}(\delta)} u_{\epsilon}^{2} d v o l_{g}=\sum_{P \in C_{\min }} \int_{B_{P}(\delta)}(\phi-\phi(P)) u_{\epsilon}^{2} d v o l_{g}+\int_{V-\cup_{P \in C_{\min }} B_{P}(\delta)} \phi u_{\epsilon}^{2} d v o l_{g}
$$

By the continuity of $\phi$, given an $\eta>0$, one can find a $\delta(\eta)>0$ such that $|\phi(x)-\phi(P)| \leq \eta$ if $\mathrm{x} \in B_{P}(\delta(\eta))$, for all $\mathrm{i}$. Hence with $\mathrm{N}=$ card $\mathrm{C}_{\min }$ :

$$
\left|\sum_{P \in C_{\min }} \int_{B_{P}(\delta(\eta))}(\phi-\phi(P)) u_{\epsilon}^{2} d v o l_{g}\right| \leq N \eta
$$

Relation (12) implies that:

$$
\lim _{\varepsilon->0} \int_{V-\cup_{P \in C_{\min }} B_{P}(\delta(\eta))} \phi u_{\epsilon}^{2} d v o l_{g}=0
$$

After choosing a subsequence of $\left\{\varepsilon_{n} \mid n \in \mathbb{N}\right\}$, still called $\left\{\varepsilon_{n} \mid n \in \mathbb{N}\right\}$, if necessary, we can assume that all the limits $\lim _{n \rightarrow \infty} \int_{V} \phi u_{\epsilon_{n}}^{2} d v o l_{g}, \lim _{n \rightarrow>\infty} \int_{B_{P}(\delta(\eta))} u_{\epsilon_{n}}^{2} d v o l_{g}, P \in C_{\text {min }}$, exist. Relation (14) implies that after choosing a subsequence of:

$$
\lim _{n \rightarrow>\infty}\left|\int_{V} \phi u_{\epsilon_{n}}^{2} d v o l_{g}-\phi(P) \sum_{i} \lim _{n \rightarrow>\infty} \int_{B_{P}(\delta(\eta))} u_{\epsilon_{n}}^{2} d v o l_{g}\right| \leq N \eta .
$$


Now note that $\lim _{n \rightarrow>\infty} \int_{B_{P}(\delta)} u_{\epsilon_{n}}^{2}$ does not depend on $\delta$.Let $\delta_{1}, \delta_{2}, \delta_{1} \leq \delta_{2}$. Then

$$
\int_{B_{P}\left(\delta_{2}\right)} u_{\epsilon_{n}}^{2} d v o l_{g}=\int_{B_{P}\left(\delta_{1}\right)} u_{\epsilon_{n}}^{2} d v o l_{g}+\int_{B_{P}\left(\delta_{2}\right)-B_{P}\left(\delta_{1}\right)} u_{\epsilon_{n}}^{2} d v o l_{g}
$$

Relation (12) implies that:

$$
\lim _{n \rightarrow>\infty} \int_{B_{P}\left(\delta_{2}\right)-B_{P}\left(\delta_{1}\right)} u_{\epsilon_{n}}^{2} d v o l_{g}=0
$$

Hence if one of the limits $\lim _{n->\infty} \int_{B_{P_{i}}\left(\delta_{1}\right)} u_{\epsilon_{n}}^{2} d v o l_{g}, \lim _{n->\infty} \int_{B_{P_{i}}\left(\delta_{2}\right)} u_{\epsilon_{n}}^{2} d v o l_{g}$ exists, so does the other and is equal to it. Set:

$$
\lim _{n->\infty} \int_{B_{P}(\delta(\eta))} u_{\epsilon_{n}}^{2} d v o l_{g}=\gamma_{P_{i}}
$$

Relation (15) implies that for any $\eta>0$ :

$$
\begin{gathered}
\left|\lim _{n->\infty} \int_{V} \phi u_{\epsilon_{n}}^{2} d v o l_{g}-\sum_{i} \phi(P) \gamma_{P}\right| \leq N \eta \\
\lim _{n->\infty} \int_{V} \phi u_{\epsilon_{n}}^{2} d v o l_{g}=\sum_{i} \phi(P) \gamma_{P}
\end{gathered}
$$

$\phi \in C(V)$ being arbitrary, (16a) shows that the sequence of measures $\mathrm{u}_{\varepsilon_{n}} \operatorname{vol}_{g}$ converges weakly (in the measure sense) to the measure $\sum_{P \in C_{\min }} \gamma_{P} \delta_{P}$. Finally note that if we apply (16a) to the constant function 1, we get:

$$
\sum_{P \in C_{\min }} \gamma_{P}=1
$$

Now we prove that $\sup _{V} u_{\epsilon}$ diverges to infinity. Because $\int_{V} u_{\epsilon}$ tends to zero, if $\underset{V}{\sup } u_{\epsilon}$ were bounded, then using the following inequality, we get a contradiction with

$$
1=\int_{V} u_{\epsilon}^{2} \leq \sup _{V} u_{\epsilon} \int_{V} u_{\epsilon}
$$

as the right-hand side would converge to zero. Let $P_{\epsilon}$ be a maximum point of $u_{\epsilon}$. Because the manifold is compact, it is possible to find a subsequence of $P_{\epsilon}$ which converges to a point $P$. centered at the point $P . v_{\epsilon}(x)=\frac{u_{\epsilon}\left(\sqrt{\epsilon} x+P_{\epsilon}\right)}{\sup u_{\epsilon}}$ and $g_{\epsilon}$ denotes the rescaled metric, then the At a maximum point $P_{\epsilon}$, using the maximum principle, $c\left(P_{\epsilon}\right) \leq \lambda_{\epsilon}$. Since $\lambda_{\epsilon}$ converges to the minimum of $v$, at the limit, $c(P) \leq \min c$. This proves that $\mathrm{P}$ is a minimum point. Using the fact that $P$ is a nondegenerate minimum point, $d\left(P_{\epsilon}, P\right) \leq$ $C \epsilon^{1 / 2}$.

We will use the following definition. 
Definition 1 The coefficient $\gamma_{P}$ is given by, for all $\delta$ small enough,

$$
\gamma_{P}=\lim _{n \rightarrow \infty} \int_{B_{P}(\delta)} u_{\epsilon_{n}}^{2} d v o l_{g}
$$

is called the concentration coefficient or the weight of the limit measure at point $P \in C_{\min }$.

The coefficient depends on the subsequence, but not on $\delta$. This coefficient characterizes the concentration measure at point $P$.

\subsection{The first eigenvalue problem for the gradient case}

We consider the limits of the first eigenfunctions as $\epsilon$ goes to zero when the vector field $b$ is the gradient of a Morse function. We establish a result similar to the one obtained in the last paragraph : when $\epsilon$ converges to zero the limits of first eigenfunctions in the weak topology of measures concentrate at the critical points of the field $b$.

Consider a Morse function $\phi$ and the vector field, $b=\nabla \phi$ and a function $c$ chosen such that the eigenvalue $\lambda_{\epsilon}$ of the operator $\epsilon \Delta_{g}+\langle b, \nabla\rangle+$.$c is positive on the manifold. To$ study the solutions of the PDE

$$
\epsilon \Delta_{g} u_{\epsilon}+<b, \nabla u_{\epsilon}>+c u_{\epsilon}=\lambda_{\epsilon} u_{\epsilon}, \text { on } V
$$

we use the transformation $b=\nabla \phi=-2 \epsilon \nabla \ln \psi_{\epsilon}$ (it is defined up to a constant) and consider the new variable $v_{\epsilon}=u_{\epsilon} \psi_{\epsilon}$. Equation (17) is transformed into the following PDE where the first order term disappeared.

$$
\epsilon^{2} \Delta_{g} v_{\epsilon}+c_{\epsilon} v_{\epsilon}=\epsilon \lambda_{\epsilon} v_{\epsilon}, \text { on } V
$$

where $c_{\epsilon}=c \epsilon+\frac{\epsilon \Delta \phi}{2}+\frac{(\nabla \phi)^{2}}{4}$.

Using the theorem of the preliminary section, we obtain the following results :

Proposition 1 Suppose that the following condition is satisfied: at the critical points $P$ of the function $\phi, c(P)+\Delta \phi(P) / 2 \geq 0$. Let $v_{\epsilon}$ be a minimizer of the following variational problem

$$
\epsilon \lambda_{\epsilon}=\inf _{v \in H^{1}\left(V_{n}\right)-\{0\}} \frac{\epsilon^{2} \int_{V}\left[\|\nabla v\|_{g}^{2}+c_{\epsilon} v^{2}\right] d v o l_{g}}{\int_{V} v^{2} d v o l_{g}}
$$

then $\lim _{\epsilon \rightarrow 0} \epsilon \lambda_{\epsilon}=\min _{V}\|\nabla \phi\|_{g}^{2}=0$. The weak limits of the normalized measures $\frac{e^{-\phi / \epsilon} v_{\epsilon}^{2} d v o l_{g}}{\int_{V} e^{-\phi / \epsilon} v_{\epsilon}^{2} d v o l_{g}}$ have their support in the set of critical points of $\phi . \sup _{V} v_{\epsilon}$ tends to $+\infty$ as $\epsilon$ goes to zero.

Proof. The proof is very similar to the proof of Theorem [1] Considering the one parameter family of eigenfunctions, we obtain that:

$$
\lim _{\epsilon \rightarrow 0} \frac{\int_{V} e^{-\phi / \epsilon} u_{\epsilon}^{2} c_{\epsilon} d v o l_{g}}{\int_{V} e^{-\phi / \epsilon} u_{\epsilon}^{2} d v o l_{g}}=\min _{V}\|\nabla \phi\|_{g}^{2}=0
$$


and for all function $\psi \in C(V)$ we have

$$
\lim _{\epsilon \rightarrow 0} \frac{\int_{V} e^{-\phi / \epsilon} u_{\epsilon}^{2} \psi d v o l_{g}}{\int_{V} e^{-\phi / \epsilon} u_{\epsilon}^{2} d v o l_{g}}=\sum_{i=1}\left\{\gamma_{P_{i}} \psi(P) \mid \mathrm{P} \in \operatorname{sing}(b)\right\}
$$

where the concentration coefficient $\gamma_{P_{i}}$ is now defined by

$$
\gamma_{P_{i}}=\lim _{\epsilon \rightarrow 0} \frac{\int_{B_{P_{i}}(\delta)} e^{-\frac{\phi}{\varepsilon}} u_{\epsilon}^{2} d v o l_{g}}{\int_{V} e^{-\frac{\phi}{\varepsilon}} u_{\epsilon}^{2} d v o l_{g}}
$$

(the limit is independent of $\delta$ ). The measure $\frac{e^{-\phi / \epsilon} u_{\epsilon}^{2} d v o l_{g}}{\int_{V} e^{-\phi / \epsilon} u_{\epsilon}^{2} d v o l_{g}}$ converges weakly to $\sum_{i=1}^{m} c_{i}^{2} \delta_{P_{i}}$ where $P_{i}$ are the critical points of the function $\phi$ or the zeros of the vector field $b=\nabla \phi$. The proof follows exactly the same steps of the previous theorem.

\section{Remark.}

The gradient case teaches two things: one is that the concentration occurs on some specific sets, related to the vector field and not to $c$ and second that the role of $c$ is to select the subset of concentration.

\subsection{The radial case : an example}

In this paragraph we give an example where the recurrent sets of the vector field consists of a limit cycle and the sequence of eigenfunction concentrates along this limit cycle. In fact, we obtain in presence of radial symmetry a uniform distribution for the limit.

Consider an annulus $A$ of $\mathbb{R}^{n}\left(A=\left\{x \in \mathbb{R}^{n} \mid 1 / 2<\|x\|_{\mathbb{R}^{n}}<3 / 2\right\}\right)$, and the radial function $u_{\epsilon}$, solution of the partial differential equation

$$
\begin{gathered}
\epsilon \Delta_{g} u_{\epsilon}+<b, \nabla u_{\epsilon}>+c u_{\epsilon}=\lambda_{\epsilon} u_{\epsilon}, \text { on } A \\
u_{\epsilon}=0, \text { on the boundary } \partial A,
\end{gathered}
$$

where the field $b$ is given by :

$$
\begin{aligned}
& b_{r}=(1-r) \\
& b_{\theta}=1
\end{aligned}
$$

and the function $a$ is radial and positive. The field $b$ has an attractive limit cycle at $r=1$. The problem reduces to:

$$
\begin{gathered}
\epsilon\left(-\partial_{r r} u_{\epsilon}-\frac{\partial_{r} u_{\epsilon}}{r}\right)+b_{r} . \partial_{r} u_{\epsilon}+a u_{\epsilon}=\lambda_{\epsilon} u_{\epsilon}, \text { on } A \\
u_{\epsilon}=0, \text { on } \partial A
\end{gathered}
$$

$b_{r}$ is the gradient of a function of $r$. Hence the results of the previous paragraph can be applied here. The presence of a boundary does not invalidate these results because the limit cycle is an attractor. As $\epsilon$ tends to zero, $u_{\epsilon}$ tends to a limit entirely supported by the limit cycle (see also Friedman [2]). 


\section{Blow up analysis with no vector fields}

In the next sections, the limit measures are analyzed using a blow-up procedure. We shall prove that as $\varepsilon$ goes to 0 , the eigenfunctions blow up in the neighborhood of some points that are determined by the potential $c$ and the vector field $b$. The speed with which these eigenfunctions blow up can also be determined when $b=0$ or when $b$ is a gradient field, using the Lyapunov functions associated to the field.

The results differ substantially in the two cases. It appears that the correct scaling is not the same in the case when there is only a potential $c$ and the case where there are a potential $c$ and a vector field $b$.

The general case, where the field can have recurrent sets of integer dimension $n \geq 1$ will be considered elsewhere. The concentration phenomenon is much more complicated, depending on the set and on the chose of Lyapunov function. More important, it cannot be studied by variational techniques, see [14.

In this section we determine exactly all the possible limits of the eigenfunctions as $\varepsilon$ tends to 0 , when there is no field. The main result says that the limit measure is concentrated on a subset of the minimum point of the potential $c$. This limit set is useful in the study in the small noise limit, the movement of a random particle moving on a Riemannian manifold in the presence of a killing potential c [15].

Moreover, we can explain the assumption 4, p.93 made by B. Simon (30 ) to study the double-well potential problem when $\epsilon$ is small. The blow-up method provides a method for the explicit computation of the concentration near a bottom well. This generalizes also the results obtained in part 9 of [5] about the concentration of the eigenfunctions in the case of $\mathbb{R}^{m}$.

Let us recall the eigenfunction problem,

$$
\begin{gathered}
\epsilon \Delta_{g} u_{\epsilon}+c u_{\epsilon}=\lambda_{\epsilon} u_{\epsilon} \\
\int_{V_{n}} u_{\epsilon}^{2} d v o l_{g}=1,
\end{gathered}
$$

$c$ is a Morse function and $C_{\text {min }}$, denotes the subset of minimal points. Recall the quotient

$$
Q_{\epsilon}(v)=\frac{\int_{V}\left[\epsilon\|\nabla u\|_{g}^{2}+c u^{2}\right] d v o l_{g}}{\int_{V} u^{2} d v o l_{g}} .
$$

We shall now state and prove the main theorems of section 2. We introduce some concepts which will be used in the proofs of these theorems.

Now a few words about the blow-up procedure. For each $P \in C_{\text {min }}$, we choose a normal coordinate system $\left(x_{1}, \ldots x_{m}\right): U_{P} \longrightarrow \mathbb{R}$, centered at $P$, defined on a domain $U_{P}$ such that:

1. $x_{1} \times \ldots \times x_{m}\left(U_{P}\right)$ contains the closed ball $B_{P}(\delta)$ centered at $P$ and having radius $\delta>0$.

2. for all $\mathrm{i}, \mathrm{j}, 1 \leq \mathrm{i}, \mathrm{j} \leq \mathrm{m}, \frac{\partial^{2} c}{\partial x_{i} \partial x_{j}}(P)=\lambda_{i}(P) \delta_{i j}$. 
3. $U_{P} \cap U_{Q}=\varnothing$ for all $P, Q \in C_{\min }, P \neq Q$.

In the following we will identifie $U_{P}$ with the open neighborhood of 0 in $\mathbb{R}^{m}, x_{1} \times \ldots \times x_{m}\left(U_{P}\right)$. For $\mathrm{r}$ such that $B_{P}(\mathrm{r}) \subset U_{P}, B_{P}(\mathrm{r})$ will denote both the geodesic ball centered at $P$ and of radius $\mathrm{r}$ in $V$ or its image by the mapping $x_{1} \times \ldots \times x_{m}$. For the sake of streamlining the notations, we shall commit the abuse while working with the coordinate system $\left(x_{1}\right.$, $\left.\ldots x_{m}\right): U_{P} \longrightarrow \mathbb{R}$, of denoting by $B_{P}(\mathrm{r})$ the ball of center $\mathrm{O}$ and radius $\mathrm{r}$ whatever the value of $\mathrm{r}(\geq 0$ of course $)$. On a manifold $\mathrm{V}$ let $\mathrm{P}$ be a point and a chart $\left(U, x_{1}, \ldots x_{m}\right)$ of $\mathrm{V}$ centered at $P: x_{i}(P)=0,1 \leq i \leq m$. The blow up of power $\mathrm{t}>0$ associated to $\mathrm{P}$ and the chart $\left(U, x_{1}, \ldots x_{m}\right)$ is the diffeomorphism $B l_{t}: U \rightarrow \mathbb{R}^{m}, B l_{t}(\mathrm{Q})=\left(\frac{1}{t} x_{1}(Q), \ldots, \frac{1}{t} x_{m}(Q)\right)$. All functions, tensors, differential operators can the be transported to the open subset $B l_{t}(U)$. Suitably normalized by a power of $\mathrm{t}$, they will have limits when $\mathrm{t}$ goes to 0 which will be defined on $\mathbb{R}^{m}$. These limits contain a trove of information about the behaviour of the original objects in the neighborhood (more precisely in the infinitesimal neighborhood) of P. To simplify the notations we shall write: $\frac{1}{t} Q$ instead of $B l_{t}(Q), \frac{1}{t} A$ instead of $B l_{t}(A)$ if $A$ is a subset of $\mathrm{U}$ and so on.

In the following all the blow-ups will be associated to geodesic charts $\left(U, x_{1}, \ldots x_{m}\right)$ with pole at $P$. On the magnified set $\frac{1}{\sqrt[4]{\varepsilon}} x_{1} \times \ldots \times x_{m}\left(U_{P}\right)$, we can define the function $\mathrm{w}_{P, \varepsilon}$ blow up of the function $\frac{v_{\varepsilon}}{v_{\varepsilon}}$ :

$$
w_{P, \varepsilon}(y)=\frac{u_{\varepsilon}(y \sqrt[4]{\varepsilon})}{\bar{u}_{\varepsilon}}
$$

where $\bar{u}_{\varepsilon}=\max _{V} \mathrm{u}_{\varepsilon}$.

\subsection{Main theorem}

Definition 2 We define $\Lambda$ as

$$
\Lambda=\inf \left[\sum_{n=1}^{m} \sqrt{\lambda_{n}(R)} \mid R \in C_{\min }\right]
$$

\section{Theorem 2 Selection-Concentration.}

- (i)For any $P \in C_{\min }$, any sequence of $v_{\varepsilon}$ 's, with $\varepsilon$ tending to 0 , contains a subsequence $\left\{u_{\varepsilon_{n}}\right\}$, such that the sequence of blown-up functions $w_{P, \varepsilon_{n}}$ at $P$ converges to a function $w_{P}: \mathbb{R}^{m} \rightarrow \mathbb{R}_{+}$, both in the $L^{2}$ norm and the $C^{\infty}$ topology.

- (ii) w satisfies the equation and inequality:

$$
\Delta_{E} w+\sum_{i=1}^{m} \lambda_{i}(P) x_{i}^{2} w=\lambda w
$$




$$
0<w \leq \max _{\mathbb{R}^{m}} w \leq 1
$$

where $\Delta_{E}$ is the negative standard Euclidean Laplacian on $\mathbb{R}^{m}$.

- (iii) If $\sum_{n=1}^{m} \sqrt{\lambda_{n}(P)}>\Lambda$ : then

$$
w_{P}=0
$$

- (iv) If $\sum_{n=1}^{m} \sqrt{\lambda_{n}(P)}=\Lambda$ and there exists a sequence $S \subset \mathbb{N}$, such that each $u_{\varepsilon_{n}}, n \in S$, has a maximum point $Q_{n}$ with the property that the sequence $\left\{Q_{n} \mid n \in S\right\}$ converges to $P$, then this function $w_{P}$ is:

$$
w_{P}(x)=\prod_{n=1}^{m} \exp \left(-\frac{x_{n}^{2} \sqrt{\lambda_{n}(P)}}{2}\right)
$$

and

$$
\lambda=\Lambda
$$

- (v) If $\sum_{n=1}^{m} \sqrt{\lambda_{n}(P)}=\Lambda$ and no such subsequence $S$ exists, then

$$
\begin{aligned}
\lambda & =\Lambda \\
w_{P} & =f_{P} \prod_{n=1}^{m} \exp \left(-\frac{x_{n}^{2} \sqrt{\lambda_{n}(P)}}{2}\right)
\end{aligned}
$$

where $f_{P}$ is a factor $\geq 0$, which depends on the sequence $\left\{u_{\varepsilon_{n}}\right\}$.

- (vi) For any sequence $\epsilon^{\prime}$, there exists at least one $P \in C_{\min }$ and at least one subsequence $S$ for which the case (iv) occurs.

Remark. Note that in case (iv) the limit $\mathrm{w}$ is independent of the sequence $\left\{\mathrm{u}_{\varepsilon_{n}}\right\}$.

In order to prove the main theorem, we need two propositions. The first gives estimates of the first eigenvalue and the second, estimates of the decay of the eigenfunctions.

\subsection{Auxiliary propositions}

Proposition 2 The first eigenvalue $\lambda_{\epsilon}$ satisfies the following inequality

$$
\min _{V} c \leq \lambda_{\epsilon} \leq \min _{V} c+\Lambda \epsilon^{1 / 2}
$$

where $\Lambda=\inf \left[\sum_{n=1}^{m} \sqrt{\lambda_{n}(P)} \mid P \in C_{\text {min }}\right]$. 
Proof. For all $\mathrm{u} \in \mathrm{H}^{1}(\mathrm{~V}), Q_{\epsilon}(u) \geq \min _{V} c \int_{V} u^{2}$. Hence $\min _{V} \leq \leq \lambda_{\epsilon}$. To prove the right hand-side inequality, we will use a test function in the variational quotient $Q$. In the neighborhood of a point $P \in C_{\min }$, consider the function:

$$
\begin{aligned}
\phi_{\epsilon} & =e^{-\sum \mu_{i} x_{i}^{2} / 2}-e^{-\frac{\rho}{2 \sqrt{\varepsilon}}}, \text { on } \mathcal{N}_{P}(\rho) \\
& =0, \text { on } V-\mathcal{N}_{P}(\rho)
\end{aligned}
$$

$\mathcal{N}_{P}(\rho)$ is the connected component of the set $\left\{\mathrm{x} \mid \sum_{i=1}^{m} \sqrt{\lambda_{i}} x_{i}^{2} \leq \rho\right\}$ containing 0 , where $\rho$ is taken so small that $\mathcal{N}_{P}(\rho)$ is contained in $\mathrm{B}_{P}(\delta)$. We take as coefficients $\mu_{i}=\sqrt{\frac{\lambda_{i}}{\epsilon}}$ where $\lambda_{i}=\lambda_{i}(P)$ for simplicity. In the coordinate system at $P$,

$$
\begin{aligned}
& g_{i j}(P)=\delta_{i j}+O\left(\|x\|_{\mathbb{R}^{m}}^{2}\right), \\
& \sqrt{\operatorname{det} g}=1-\sum_{i, j=1}^{m} \frac{R i c_{i j}}{6} x^{i} x^{j}+O\left(\|x\|_{\mathbb{R}^{m}}^{3}\right),
\end{aligned}
$$

Ric denotes the Ricci tensor. We denote $\prod_{1}^{m} \mu_{i}$ by $\mu$ and the quadratic form $\sum \mu_{i}\left(x^{i}\right)^{2}$ by $q$. We recall that:

$$
\begin{aligned}
\int_{\mathbb{R}} e^{-\mu_{i} x^{2}} d x & =\sqrt{\frac{\pi}{\mu_{i}}}, \\
\int_{\mathbb{R}} x^{2} e^{-\mu_{i} x^{2}} d x & =\frac{\sqrt{\pi}}{2 \mu_{i}^{3 / 2}} .
\end{aligned}
$$

To evaluate the quotient $Q_{\varepsilon}\left(u_{\varepsilon}\right)$, we compute the leading terms in $\epsilon$ of the integrals $\mu$

$$
\begin{gathered}
\int_{\mathcal{N}_{P}(\rho)}\left\|\nabla \phi_{\epsilon}\right\|_{g}^{2} d v o l_{g} \\
\int_{\mathcal{N}_{P(\rho)}} c \phi_{\epsilon}^{2} d v o l_{g} \\
\int_{\mathcal{N}_{P}(\rho)}\left(\left\|\nabla \phi_{\epsilon}\right\|_{g}^{2}\right) d v o l_{g}=\int_{\mathcal{N}_{P}(\rho)} \sum_{i, j=1}^{m} g^{i j} \frac{\partial \phi_{\varepsilon}}{\partial x_{i}} \frac{\partial \phi_{\varepsilon}}{\partial x_{j}} \sqrt{\operatorname{det} g} d x
\end{gathered}
$$

where $g^{i j}$ is the matrix inverse of $\mathrm{g}_{i j}$ and $\mathrm{dx}$ is the Lebesgue volume.

$$
\int_{\mathcal{N}_{P}(\rho)}\left(\left\|\nabla \phi_{\epsilon}\right\|_{g}^{2}\right) d v o l_{g}=\int_{\mathcal{N}_{P}(\rho)}\left[\sum_{i=1}^{m} \mu_{i}^{2} x_{i}^{2}+\sum_{i, j, k=1}^{m} a_{i j k} x_{i} x_{j} x_{k}\right] e^{-q} d x
$$

where the functions $a_{i j k}$ are defined $\mathrm{C}^{\infty}$ and bounded on $B_{P}(\delta)$. Performing the blow-up at $P$ i.e. the change of variable $z_{i}=x_{i} \sqrt{\mu_{i}}$,

$$
\int_{\mathcal{N}_{P}(\rho)}\left(\left\|\nabla \phi_{\epsilon}\right\|_{g}^{2}\right) d v o l_{g}=\int_{B\left(0, \frac{\rho}{\sqrt[4]{\varepsilon}}\right)}\left[\sum_{i, j=1}^{m} \mu_{i} z_{i}^{2}+\sum_{i, j, k=1}^{m} a_{i j k}(x) \frac{z_{i} z_{j} z_{k}}{\sqrt{\mu_{i} \mu_{j} \mu_{k}}}\right] \exp \left(-\|z\|_{\mathbb{R}^{m}}^{2}\right) \frac{d z}{\sqrt{\mu}}
$$


where $\mu=\prod_{i=1}^{m} \mu_{i}$.

$$
\varepsilon \int_{\mathcal{N}_{P}(\rho)}\left(\left\|\nabla \phi_{\epsilon}\right\|_{g}^{2}\right) d v o l_{g}=\frac{\pi^{m / 2}}{\sqrt{\mu}}\left[\sum_{i=1}^{n} \frac{\sqrt{\epsilon} \lambda_{i}}{2}+O\left(\varepsilon^{\frac{7}{4}}\right) .\right]
$$

We will now evaluate the potential term:

$$
\begin{aligned}
\int_{\mathcal{N}_{P}(\rho)} c \phi_{\epsilon}^{2} d v o l_{g} & =\int_{\mathcal{N}_{P}(\rho)} \phi_{\epsilon}^{2}\left[\left\{c(P)+\sum_{k=1}^{m} \lambda_{i} x_{i}^{2}+O\left(\|x\|_{\mathbb{R}^{m}}^{3}\right)\right\}\left(1-\frac{R i c_{i j}(P)}{6} x^{i} x^{j}+O\left(\|x\|_{\mathbb{R}^{m}}^{3}\right)\right] d x\right. \\
& =c(P) \int_{\mathcal{N}_{P}(\rho)} \phi_{\epsilon}^{2} d x+\int_{\mathcal{N}_{P}(\rho)} \phi_{\epsilon}^{2}\left[\sum_{k=1}^{m}\left(\lambda_{i}-\frac{c(P) R i c_{i i}(P)}{6}\right) x_{i}^{2}+\sum_{i, j, k=1}^{m} b_{i j k} x_{i} x_{j} x_{k}\right] d x
\end{aligned}
$$

where the functions $\mathrm{a}_{i j k}$ are defined $\mathrm{C}^{\infty}$ and bounded on $\mathrm{B}(\delta)$. With the same change of variables, after expanding the square $u_{\epsilon}^{2}(x)=e^{-q}-2 e^{-q / 2-\rho / 2 \sqrt{\varepsilon}}+e^{-\rho / \sqrt{\varepsilon}}$ we get:

$$
\int_{\mathcal{N}_{P}(\rho)} x_{i}^{2} \phi_{\epsilon}^{2} d x=\frac{\pi^{m / 2}}{2 \sqrt{\mu}} \sqrt{\frac{\varepsilon}{\lambda_{i}(P)}}+O_{w}\left(e^{-\rho / 2 \sqrt{\epsilon}}\right) .
$$

$\mathrm{O}_{w}\left(\exp -\frac{\rho}{\sqrt{\varepsilon}}\right)$ means that for any $\eta \in[0,1[$, there exists a constant $\mathrm{K}(\eta)$ independant of $\varepsilon$ sucht that the error is at most equal in absolute value to $K(\eta) \exp \left[-\frac{\eta \rho}{\sqrt{\varepsilon}}\right]$. An easy symmetry argument shows that:

$$
\int_{\mathcal{N}_{P}(\rho)} x_{i} x_{j} \phi_{\epsilon}^{2} d x=0 \text { if } \mathrm{i} \neq \mathrm{j}
$$

The potential term in the integral becomes:

$$
\int_{\mathcal{N}_{P}(\rho)} c \phi_{\epsilon}^{2}=\frac{\pi^{m / 2}}{\sqrt{\mu}}\left(c(P)+\frac{1}{2} \sum_{i=1}^{m}\left(\lambda_{i}-c(P) \frac{R i c_{i i}(P)}{6}\right) \sqrt{\frac{\epsilon}{\lambda_{i}}}+O\left(\varepsilon^{\frac{3}{2}}\right)\right)
$$

Also:

$$
\int_{V} \phi_{\varepsilon}^{2} d v o l_{g}=1-\sum_{i=1}^{m} \frac{\operatorname{Ric}_{i i}(P)}{12} \sqrt{\frac{\epsilon}{\lambda_{i}}}+O_{w}\left(e^{-\rho / 2 \epsilon^{1 / 2}}\right)
$$

The quotient can now be evaluated:

$$
\begin{gathered}
Q_{\epsilon}(u)=\frac{\epsilon \int_{V}\left[\|\nabla u\|_{g}^{2}+c u^{2}\right] d v o l_{g}}{\int_{V} u^{2} d v o l_{g}} \\
Q_{\varepsilon}\left(\phi_{\varepsilon}\right)=\frac{\frac{\pi^{m / 2}}{\sqrt{\mu}}\left(\sum_{i=1}^{m} \frac{\sqrt{\varepsilon \lambda_{i}}}{2}+c(P)+\sum_{i=1}^{m}\left(\lambda_{i}-c(P) \frac{R i c_{i i}(P)}{6}\right) \sqrt{\frac{\epsilon}{\lambda_{i}}}+O\left(\varepsilon^{\frac{3}{2}}\right)\right)}{\frac{\pi^{m / 2}}{\sqrt{\mu}}+\sum_{i=1}^{m} \frac{R i c_{i i}(P)}{6} \sqrt{\frac{\epsilon}{\lambda_{i}}}+o\left(e^{-\rho / 4 \epsilon^{1 / 2}}\right)}
\end{gathered}
$$




$$
Q_{\varepsilon}\left(\phi_{\varepsilon}\right)=\frac{\left.c(P)\left[1-\sum_{i=1}^{m} \frac{R i c_{i i}(P)}{12} \sqrt{\frac{\epsilon}{\lambda_{i}}}\right]+\sum_{i=1}^{m} \sqrt{\varepsilon \lambda_{i}}+O\left(\varepsilon^{\frac{3}{2}}\right)\right)}{1-\sum_{i=1}^{m} \frac{R i c_{i i}(P)}{12} \sqrt{\frac{\epsilon}{\lambda_{i}}}+O_{w}\left(e^{-\rho / 2 \epsilon^{1 / 2}}\right)}
$$

If we use the fact that $c(P)=\min _{V} c$, the quotient can be simplified as follows

$$
Q_{\epsilon}\left(\phi_{\varepsilon}\right)=\min _{V} c+\frac{\sum_{i=1}^{m} \sqrt{\lambda_{i} \epsilon}+O\left(\varepsilon^{\frac{3}{2}}\right)}{1+\sum_{i=1}^{m} \frac{R i c_{i i}(P)}{12} \sqrt{\frac{\epsilon}{\lambda_{i}}}+O_{w}\left(e^{-\rho / 2 \epsilon^{1 / 2}}\right)}
$$

If we take the minimum over all test functions centered at any critical points of the set of minimal points $C_{m}$, we obtain the estimate:

$\min _{V} c \leq \lambda_{\epsilon} \leq Q_{\epsilon}\left(\phi_{\varepsilon}\right) \leq \min _{V} c+\inf \left\{\sum_{i=1}^{m} \sqrt{\lambda_{i}(P) \epsilon} \mid P \in C_{\min }\right\}+O(\varepsilon) \leq \min _{V} c+\Lambda \epsilon^{1 / 2}+O(\varepsilon)$.

Remark: We expect in general for smooth potential that there exists an asymptotic expansion:

$$
\lambda_{\epsilon}=\sum_{k=0}^{n} c_{k} \epsilon^{k / 2}+o\left(\epsilon^{n / 2}\right)
$$

If it does exist can one find a systematic procedure to compute the coefficients $\mathrm{c}_{k}$ ? From the previous result, we have that

$$
c_{0}=\min c
$$

which is the Topological Pressure. We will see in the following results that

$$
c_{1}=\inf \left\{\sum_{i=1}^{m} \sqrt{\lambda_{i}(P)} \mid P \in C_{\min }\right\} .
$$

Proposition 2 provides an estimate of the velocity of convergence of the sequence of maximum points $Q_{\epsilon}$ of $\mathrm{u}_{\varepsilon}$ to an element of $C_{\min }$.

Lemma 1 (i)If for a sequence $\epsilon_{n}$ tending to zero, $\lim _{n \rightarrow \infty} \int_{V} u_{\epsilon_{n}}^{2} d v o l_{g}>0$, then

$$
\lim _{n \rightarrow \infty} \sup _{V} u_{\epsilon_{n}}=+\infty
$$

Proof. (i)Suppose that for a subsequence $\varepsilon_{n}$, still denoted by $\varepsilon_{n}, \lim _{n \rightarrow \infty} \sup _{V} u_{\epsilon_{n}}<+\infty$. Then for all $\mathrm{n}, \sup _{V} u_{\epsilon_{n}} \leq N$, a constant. For any $\eta>0$, chose an open neighborhood $K$ of $C_{\text {min }}$, such that $\operatorname{vol}_{g}(K)$ is smaller than $\frac{\eta}{2 N}$. Now

$$
\int_{V} u_{\epsilon_{n}}^{2} d v o l_{g}=\int_{K} u_{\epsilon_{n}}^{2} d v o l_{g}+\int_{V-K} u_{\epsilon_{n}}^{2} d v o l_{g}
$$




$$
\int_{V} u_{\epsilon_{n}}^{2} d v o l_{g}=\int_{K} u_{\epsilon_{n}}^{2} d v o l_{g}+\sup _{V} u_{\epsilon_{n}} \int_{V-K} \frac{u_{\epsilon_{n}}^{2}}{\sup u_{\epsilon_{n}}} d v o l_{g}
$$

By Appendix II, $\frac{u_{\epsilon_{n}}^{2}}{\sup _{V} u_{\epsilon_{n}}}>0$ uniformly on $V-K$. Hence $\lim _{n \rightarrow \infty} \int_{V} u_{\epsilon_{n}}^{2} d v o l_{g} \leq \eta$. Because $\eta$ is arbitrary, $\lim _{n \rightarrow \infty} \int_{V} u_{\epsilon_{n}}^{2} d v o l_{g}=0$. A contradiction.

Recall that $d_{g}$ denotes the Riemmanian distance associated to the metric $g$,

Lemma 2 For each $\varepsilon$, let us denote by $\mathcal{M}_{\varepsilon}$ the set of all maximum points of $v_{\varepsilon}$. There exists a constant $A$ depending only on $c$, such that:

$$
\sup _{q \in \mathcal{M}_{\varepsilon}} d_{g}^{2}\left(q, C_{\min }\right) \leq A \epsilon^{1 / 2}
$$

It follows from this that the set of limit points of the set of maximum points of $v_{\varepsilon}$ is contained in $C_{\min }$.

Proof. Let $\mathrm{q} \in \mathcal{M}_{\varepsilon}$. Recall $\epsilon \Delta_{g} u_{\epsilon}(q)+c(q) u_{\epsilon}(q)=\lambda_{\epsilon} u_{\epsilon}(q)$. Because the solution $\mathrm{u}_{\varepsilon}$ is positive and $\Delta_{g} u_{\epsilon}(q) \geq 0$, at the maximum point q of $u_{\varepsilon}, c(q) \leq \lambda_{\varepsilon}$. By Proposition 2, $0 \leq c(q)-\min _{V} c \leq \lambda_{\epsilon}-\min _{V} c \leq \Lambda \epsilon^{1 / 2}$. Because the critical points of $\mathrm{c}$ are non-degenerate, it is easy to see that there exists a constant $\Gamma$ depending only on $c$ such that for $P \in V$, $\mathrm{d}_{g}^{2}\left(P, C_{\min }\right) \leq \Gamma\left(c(P)-\min _{V} c\right)$. Take $A=\Gamma \Lambda$.

Remark. This result proves that any sequence of $\varepsilon^{\prime}$ s converging to 0 , contains a subsequence $\left\{\varepsilon_{k} \mid k \in \mathbb{N}\right\}$ such that there exists a $P \in C_{\min }$ and a vector $P^{*} \in T_{P} V$ with the property:

$$
P_{\epsilon}=\exp _{P}\left(\epsilon^{1 / 4} P^{*}+o\left(\epsilon^{1 / 4}\right)\right) .
$$

The length $\left\|P^{*}\right\|$ of the vector $P^{*}$ is the distance between the peak of concentration and the set $C_{\min }$ in the blow up space. It can be considered as a measure of the convergence velocity.

We have so far computed an estimate of the rescaled eigenvalue $\frac{\lambda_{\epsilon}-\min _{V_{m}} c}{\epsilon^{1 / 2}}$. Now we will provide an estimate of the eigenfunction in the neighborhood of the points in $C_{\min }$. Let $P$ be a point in $C_{\min }$. Recall that $\mathrm{w}_{\varepsilon}=\frac{u_{\varepsilon}}{u_{\varepsilon}}$ and $\overline{u_{\varepsilon}}=\max _{V} \mathrm{u}_{\varepsilon}$.

Proposition 3 For all $\left.\varepsilon_{0} \in\right] 0,1[$ :

- (i) $\sup _{\left[0, \varepsilon_{0}\right]} \int_{B_{P}(\delta / \sqrt[4]{\varepsilon})} w_{\varepsilon}(y)^{2} d y<+\infty$. More generally, for any continuous function $f:[0,1] \times \mathbb{R}^{m} \longrightarrow \mathbb{R},(\epsilon, y) \longrightarrow f(\epsilon, y)$, having at most polynomial growth at infinity,

$$
\sup _{\left.j 0, \varepsilon_{0}\right]} \int_{B_{P}(\delta / \sqrt[4]{\varepsilon})} f(\epsilon, y) w_{\varepsilon}(y)^{2} d y<+\infty
$$


- (ii) the set of restrictions $\left.\left.w_{\varepsilon}^{2} \mid B_{P}(\delta / \sqrt[4]{\varepsilon}), \varepsilon \in\right] 0,1\right]$, of the $w_{\varepsilon}^{2}$ to the balls $B_{P}(\delta / \sqrt[4]{\varepsilon})$ satisfies the following condition: for any $\eta>0$, there exists a compact $K \subset \mathbb{R}^{m}$ and $a \varepsilon(\eta)>0$ such that

$$
\int_{B_{P}(\delta / \sqrt[4]{\varepsilon})-K} f(\epsilon, y) w_{\varepsilon}(y)^{2} d y \leq \eta
$$

for all $\varepsilon \in] 0, \varepsilon(\eta)]$.

\subsection{Proof of the Proposition 3}

To start with, rewrite equation (21) as follows

$$
\sqrt{\epsilon} \Delta_{g} u_{\epsilon}+\frac{c-\min _{V_{m}} c}{\epsilon^{1 / 2}} u_{\epsilon}=\frac{\lambda_{\epsilon}-\min _{V_{m}} c}{\epsilon^{1 / 2}} u_{\epsilon}
$$

Introducing the notations $c_{\epsilon}=\frac{c-\min _{V_{m}} c}{\epsilon^{1 / 2}}$ and $\mu_{\epsilon}=\frac{\lambda_{\epsilon}-\min _{V_{m}} c}{\epsilon^{1 / 2}}$ for simplicity, we have:

$$
\sqrt{\epsilon} \Delta_{g} u_{\epsilon}+c_{\varepsilon} u_{\epsilon}=\mu_{\varepsilon} u_{\epsilon}
$$

The function $\mathrm{e}^{-t \mu_{\varepsilon}} v_{\varepsilon}(x)$ is the solution of the parabolic Cauchy problem:

$$
\begin{aligned}
\frac{\partial p}{\partial t} & =-\sqrt{\epsilon} \Delta_{g} p-c_{\epsilon} p \\
p(0, x) & =u_{\epsilon}(x) .
\end{aligned}
$$

Note that the sequence $\mu_{\epsilon}$ is bounded. We estimate $w_{\varepsilon}$ in the ball $B_{P}(\delta)$, using the fact that the restriction of the function $e^{-\mu_{\epsilon} t} v_{\epsilon}(x)$ to $\mathrm{B}_{P}(\delta)$ is the solution of the parabolic initial- boundary value problem:

$$
\begin{aligned}
\frac{\partial p}{\partial t} & =-\sqrt{\epsilon} \Delta_{g} p-c_{\epsilon} p \\
p(0, x) & =u_{\epsilon}(x) \\
p(t, x)_{\mid \partial B_{P}(\delta)} & =u_{\epsilon}(x)_{\mid \partial B_{P}(\delta)} e^{-\mu_{\epsilon} t} .
\end{aligned}
$$

In the coordinate system at $P$,

$$
\frac{\partial p}{\partial t}=\sqrt{\epsilon} \sum_{i, j=1}^{m} g^{i j} \frac{\partial^{2} p}{\partial x_{i} \partial x_{j}}-\sqrt{\epsilon} \sum_{k=1}^{m} B^{k} \frac{\partial p}{\partial x_{k}}-c_{\epsilon} p
$$

where

$$
B_{k}=-\sum_{i, j=1}^{m} g^{i j} \Gamma_{i j}^{k}
$$


For $\mathrm{x} \in B_{P}(\delta)$, the solution is given by the Feynman-Kac formula with boundary term:

$$
e^{-\mu_{\epsilon} t} u_{\epsilon}(x)=E_{x}\left(e^{-\mu_{\epsilon} t} u_{\epsilon}\left(X_{\epsilon}(t)\right) \chi_{\left(t<\tau_{\epsilon}^{x}\right)} e^{-\int_{0}^{t} c_{\epsilon}\left(X_{\epsilon}(s) d s\right)}\right)+E_{x}\left(e^{-\mu_{\epsilon} \tau_{\varepsilon}^{x}} u_{\epsilon}\left(X_{\epsilon}\left(\tau_{\varepsilon}^{x}\right)\right) \chi_{\left(t>\tau_{\epsilon}^{x}\right)} e^{-\int_{0}^{\tau_{\epsilon}^{x}} c_{\epsilon}\left(X_{\epsilon}(t)\right) d s}\right)
$$

where $X_{\varepsilon}(t)$ is the process starting at $\mathrm{x}$ at time 0 and satisfying the Itô equation:

$$
d X_{\varepsilon}(t)=\sqrt{\varepsilon} B\left(X_{\varepsilon}(t)\right)+\sqrt[4]{\varepsilon} \sigma\left(X_{\varepsilon}(t)\right) d W(t) \text { for } \mathrm{t} \leq \tau_{\varepsilon}^{U}
$$

where $W(\mathrm{t})$ is a standard $m$ dimensional Brownian motion and $\sigma: U \longrightarrow \operatorname{End}\left(\mathbb{R}^{m}\right)$ is the positive definite square root of the matrix function $\left(2 g^{i j}\right) . \tau_{\varepsilon}^{x}$ is the first exit time from the ball $B_{P}(\delta)$, of the process $X_{\varepsilon}$, starting at $\mathrm{x}$. Let $\bar{u}_{\varepsilon}=\max _{V} u_{\varepsilon}$. Then equation (22) implies the inequality:

$$
\frac{u_{\epsilon}(x)}{\overline{u_{\varepsilon}}} \leq\left[E_{x}\left(\chi_{\left(t<\tau_{\epsilon}^{x}\right)} e^{-\int_{0}^{t} c_{\epsilon}\left(X_{\epsilon}(s)\right) d s}\right)+E_{x}\left(e^{\mu_{\varepsilon}\left(t-\tau_{\varepsilon}^{x}\right)} \frac{u_{\epsilon}\left(X\left(\tau_{\varepsilon}\right)\right)}{\overline{u_{\varepsilon}}} \chi_{\left(t>\tau_{\epsilon}^{x}\right)} e^{-\int_{0}^{\tau_{\epsilon}^{x}} c_{\epsilon}\left(X_{\epsilon}(s)\right) d s}\right)\right]
$$

for $x \in B_{P}(\delta)$. Let us define:

$$
\begin{aligned}
& \mathrm{I}=e^{\mu_{\epsilon} t} E_{x}\left(\frac{u_{\epsilon}\left(X\left(\tau_{\varepsilon}\right)\right)}{\overline{u_{\varepsilon}}} \chi_{\left(t>\tau_{\epsilon}^{x}\right)} e^{-\int_{0}^{\tau_{\epsilon}^{x}} c_{\epsilon}\left(X_{\epsilon}(s)\right) d s}\right) \\
& \mathrm{II}=E_{x}\left(\chi_{\left(t<\tau_{\epsilon}^{x}\right)} e^{-\int_{0}^{t} c_{\epsilon}\left(X_{\epsilon}(s)\right) d s}\right)
\end{aligned}
$$

We estimate the terms I, II independently.

\section{Estimate of $I$}

To estimate the boundary term I in inequality (24), we apply the results proved in Appendix 2 to the equation(21) taking $\psi=c-\min _{V} c, b=0, c_{\varepsilon}=\min _{V} c-\lambda_{\varepsilon}$ and $\varepsilon=\varepsilon$. Then for any integer $\mathrm{k}$, any compact subset $\mathcal{C}$ disjoint from the set $\mathrm{C}_{\min }$, there exists a positive constant $\mathrm{A}(\mathrm{k}, \mathcal{C})$ such that for $\varepsilon \in] 0,1]$ :

$$
\max _{\mathcal{C}} u_{\epsilon} \leq A(k, \mathcal{C}) \varepsilon^{k} \bar{v}_{\varepsilon}
$$

Taking $\mathcal{C}=\mathrm{V}-\cup\left\{B_{P}(\delta) \mid P \in C_{\min }\right\}$ :

$$
\mathrm{I}=e^{\mu_{\epsilon} t} E_{x}\left(\frac{u_{\epsilon}\left(X_{\varepsilon}\left(\tau_{\varepsilon}^{x}\right)\right)}{\bar{u}_{\varepsilon}} e^{-\int_{0}^{\tau_{\epsilon}^{x}} c_{\epsilon}\left(X_{\varepsilon}(s)\right) d s}\right) \leq e^{\mu_{\epsilon} t} A(k, \delta) \epsilon^{k}
$$

\section{Estimate of $\underline{\overline{I I}}$}

Let $\alpha$ be a number in ]0, $\frac{1}{6}[$. Assume that $\varepsilon<1$. We split II as follows:

$$
\mathrm{II}=E_{x}\left(\chi_{1} \chi_{\left(t<\tau_{\epsilon}^{x}\right)} e^{-\int_{0}^{t} c_{\epsilon}\left(X_{\epsilon}(s)\right) d s}\right)+E_{x}\left(\left(1-\chi_{1}\right) \chi_{\left(t<\tau_{\epsilon}^{x}\right)} e^{-\int_{0}^{t} c_{\epsilon}\left(X_{\epsilon}(s)\right) d s}\right)
$$


where $\chi_{1}$ is the characteristic function of the $\operatorname{set}\left\{\sup _{[0, t]}\left\|X_{\varepsilon}(s)\right\|_{\mathbb{R}^{m}} \geq \delta \varepsilon^{\alpha}\right\}$.

$$
\mathrm{II}=\mathrm{III}+\mathrm{IV}
$$

Because $\mathrm{c}_{\varepsilon}$ is non negative and $\alpha<\frac{1}{6}$ :

$$
\mathrm{III}=E_{x}\left(\chi_{1} \chi_{\left(t<\tau_{\epsilon}^{x}\right)} e^{-\int_{0}^{t} c_{\epsilon}\left(X_{\epsilon}(s)\right) d s}\right) \leq P_{x}\left[\sup _{\left[0, t \wedge \tau_{\varepsilon}^{x}\right]}\left\|X_{\varepsilon}(t)\right\|_{\mathbb{R}^{m}} \geq \delta \varepsilon^{\alpha}\right]
$$

We have:

$$
X_{\varepsilon}\left(t \wedge \tau_{\varepsilon}^{x}\right)=\sqrt{\varepsilon} \int_{0}^{t \wedge \tau_{\varepsilon}^{x}} B\left(X_{\varepsilon}(s)\right)+\sqrt[4]{\varepsilon} \int_{0}^{t \wedge \tau_{\varepsilon}^{x}} \sigma\left(X_{\varepsilon}(s)\right) d W(s)
$$

Now $\sup _{\left[0, t \wedge \tau_{\varepsilon}^{x}\right]}\left\|X_{\varepsilon}(t)\right\|_{\mathbb{R}^{m}} \leq \delta$. Hence $\left\|\sqrt{\varepsilon} \int_{0}^{t \wedge \tau_{\varepsilon}^{x}} B\left(X_{\varepsilon}(s)\right)\right\|_{\mathbb{R}^{m}} \leq t M_{1} \sqrt{m \varepsilon}$, where $M_{1}=$ $\sup \|B(x)\|_{\mathbb{R}^{m}}$. Then a well known lemma (see [34]) shows that if $\delta \varepsilon^{\alpha}>\|x\|_{\mathbb{R}^{m}}+$ $x \in B(\delta)$ $t M_{1} \sqrt{m \varepsilon}$

$$
P_{x}\left[\sup _{\left[0, t \wedge \tau_{\varepsilon}^{x}\right]}\left\|X_{\varepsilon}(t)\right\|_{\mathbb{R}^{m}} \geq \delta \varepsilon^{\alpha}\right] \leq 2 m \exp -\frac{\left[\delta \varepsilon^{\alpha}-\|x\|_{\mathbb{R}^{m}}-t M_{1} \sqrt{m \varepsilon}\right]^{2}}{2 m M^{2} t \sqrt{\varepsilon}},
$$

where $M=\sup _{B_{P}(\delta)}\|\sigma(x)\|_{\mathbb{R}^{m}}$. It is clear that:

$$
E_{x}\left(\chi_{1} \chi_{\left(t<\tau_{\epsilon}^{x}\right)} e^{-\int_{0}^{t} c_{\epsilon}\left(X_{\epsilon}(s)\right) d s}\right) \leq E_{x}\left(\chi_{1} \chi_{\left(t<\tau_{\epsilon}^{x}\right)}\right) .
$$

Hence if $\|x\|_{\mathbb{R}^{m}}+t M_{1} \sqrt{\varepsilon}<\delta \varepsilon^{\alpha}$ :

$$
E_{x}\left(\chi_{1} \chi_{\left(t<\tau_{\epsilon}^{x}\right)} e^{-\int_{0}^{t} c_{\epsilon}\left(X_{\epsilon}(s)\right) d s}\right) \leq 2 m \exp -\frac{\left[\delta \varepsilon^{\alpha}-\left(\|x\|_{\mathbb{R}^{m}}+t M_{1} \sqrt{m \varepsilon}\right)\right]^{2}}{2 m M^{2} t \sqrt{\varepsilon}} .
$$

To estimate the second integral IV $=E_{x}\left(\left(1-\chi_{1}\right) \chi_{\left(t<\tau_{\epsilon}^{x}\right)} e^{-\int_{0}^{t} c_{\epsilon}\left(X_{\epsilon}(s)\right) d s}\right)$, let us define the process $Y_{\epsilon}(t)$ for $t<\tau_{\epsilon}^{U}=$ first exit time of the process $X_{\varepsilon}$ from $U_{P}$ :

$$
Y_{\epsilon}(t)=\frac{X_{\varepsilon}(t)}{\sqrt[4]{\varepsilon}}
$$

Then:

$$
\begin{gathered}
d Y_{\varepsilon}(t)=\sqrt[4]{\varepsilon} B\left(X_{\varepsilon}(t)\right)+\sigma\left(X_{\varepsilon}(t)\right) d W(t) \\
Y_{\varepsilon}(t)=Y_{\varepsilon}(0)+\sqrt[4]{\varepsilon} \int_{0}^{t \wedge \tau_{\epsilon}^{U}} B\left(X_{\varepsilon}(s)\right) d s+\int_{0}^{t \wedge \tau_{\epsilon}^{U}} \sigma\left(X_{\varepsilon}(s)\right) d W(s)
\end{gathered}
$$


for $t \leq \tau_{\varepsilon}^{U}$. But using the definition of $\sigma$ and the system of coordinates $\left(x_{1}, \ldots x_{m}\right)$ :

$$
\sigma(x)=\sqrt{2}\left(I d_{m}+\Phi(x)\right)
$$

where $\Phi: U \longrightarrow \operatorname{End}\left(\mathbb{R}^{m}\right)$ is a $C^{\infty}$ matrix function such that :

$$
\Phi_{i j}(x)=\sum_{k, l=1}^{m} \Phi_{i j k l}(x) x_{k} x_{l}
$$

Its value at $\mathrm{P}$ is:

$$
\begin{aligned}
\Phi_{i j k l}(P) & =-\frac{1}{6}\left(R_{i k l j}(P)+R_{i l k j}(P)\right), \\
\sigma\left(X_{\varepsilon}(t)\right) & =\sqrt{2}\left(I d_{m}+\sqrt{\varepsilon} \widehat{\Phi}\left(\varepsilon, Y_{\varepsilon}(t)\right)\right)
\end{aligned}
$$

where $\widehat{\Phi}\left(\varepsilon, Y_{\varepsilon}\right)$ is a matrix function such that:

$$
\widehat{\Phi}_{i j}\left(\varepsilon, Y_{\varepsilon}(t)\right)=\sum_{k, l=1}^{m} \Phi_{i j k l}\left(\sqrt[4]{\varepsilon} Y_{\varepsilon}(t)\right) Y_{\varepsilon, k}(t) Y_{\varepsilon, l}(t)
$$

Also the components of the field $B$ :

$$
B_{i}(x)=\sum_{j=1}^{m} B_{i j}(x) x_{j}
$$

Hence:

$$
\begin{aligned}
& B\left(X_{\varepsilon}(t)\right)=\sqrt[4]{\varepsilon} \widehat{B}\left(\varepsilon, Y_{\varepsilon}(t)\right) \\
& \widehat{B}_{i}(\varepsilon, y)=\sum_{j=1}^{m} B_{i j}(y \sqrt[4]{\varepsilon}) y_{j}
\end{aligned}
$$

Finally:

$$
\begin{gathered}
Y_{\varepsilon}(t)=Y_{\varepsilon}(0)+\sqrt{2} W(t)+Z_{\varepsilon}(t) \\
Z_{\varepsilon}(t)=\sqrt{\varepsilon} \int_{0}^{t \wedge \tau_{\epsilon}^{U}} \widehat{B}\left(\varepsilon, Y_{\varepsilon}(s)\right) d s+\sqrt{2 \varepsilon} \int_{0}^{t \wedge \tau_{\epsilon}^{U}} \widehat{\Phi}\left(\varepsilon, Y_{\varepsilon}(s)\right) d W(s)
\end{gathered}
$$

To estimate IV, we split it into V and VI, choosing a $\beta \in[0, \alpha[$ :

$$
\begin{gathered}
\mathrm{V}=E_{x}\left(\left(1-\chi_{1}\right) \chi_{\left(t<\tau_{\epsilon}^{x}\right)} e^{-\int_{0}^{t} c_{\epsilon}\left(X_{\epsilon}(s)\right) d s} ; \sup _{[0, t]}\left\|Z_{\varepsilon}(s)\right\|_{\mathbb{R}^{m}} \geq \varepsilon^{\beta}\right) \\
\mathrm{VI}=E_{x}\left(\left(1-\chi_{1}\right) \chi_{\left(t<\tau_{\epsilon}^{x}\right)} e^{-\int_{0}^{t} c_{\epsilon}\left(X_{\epsilon}(s)\right) d s} ; \sup _{[0, t]}\left\|Z_{\varepsilon}(s)\right\|_{\mathbb{R}^{m}}<\varepsilon^{\beta}\right)
\end{gathered}
$$




\section{Estimate of V}

We shall use a variant of the inequality (37.9) stated in (28] p.78):

$$
P\left\{\sup _{[0, t]}\left\|M_{s}\right\| \geq y ;[M]_{t} \leq K\right\} \leq 2 m \exp \left(-\frac{y^{2}}{2 m K}\right),
$$

where $M$ is a continuous local martingale, 0 at $t=0$. We apply this formula to $M_{t}=$ $Z_{\varepsilon}(t)-\sqrt[4]{\varepsilon} \int_{0}^{t} \widehat{B}\left(\varepsilon, Y_{\varepsilon}(s)\right) d s=\sqrt{2} \sqrt[4]{\varepsilon} \int_{0}^{t} \widehat{\Phi}\left(\varepsilon, Y_{\varepsilon}(s)\right) d W(s)$ stopped at $\tau_{\varepsilon}^{x}$, the exit time of the process $X_{\varepsilon}$ from $B_{P}(\delta)$.

Note that:

$$
\left(1-\chi_{1}\right)\left\|\sqrt{\varepsilon} \widehat{\Phi}\left(\varepsilon, Y_{\varepsilon}(s)\right)\right\|_{E n d\left(\mathbb{R}^{m}\right)} \leq C_{2} \delta^{2} \varepsilon^{2 \alpha}
$$

and that:

$$
\left(1-\chi_{1}\right)\left\|\sqrt{\varepsilon} \widehat{B}\left(\varepsilon, Y_{\varepsilon}(s)\right)\right\|_{\mathbb{R}^{m}} \leq C_{3} \delta \varepsilon^{\alpha+\frac{1}{4}},
$$

for some constants $C_{2}$ depending only on the values of $\sigma$ on $B_{P}(\delta), C_{3}$ depending only on the values of the vector field $B$ on $B_{P}(\delta)$. Thus we have:

$$
\left\{\sup _{[0, t]}\left\|M_{s}\right\| \geq y ; \chi_{1}=0\right\} \subset\left\{\sup _{[0, t]}\left\|M_{s}\right\| \geq y ;[M]_{t} \leq t\left(C_{2} \delta^{2} \varepsilon^{2 \alpha}\right)^{2}\right\}
$$

Recall that:

$$
\begin{aligned}
& {[M]_{t}=2 \sqrt{\varepsilon} \int_{0}^{t \wedge \tau_{x}^{\varepsilon}} \operatorname{tr}\left[\widehat{\Phi}\left(\varepsilon, Y_{\varepsilon}(s)\right) \widehat{\Phi}\left(\varepsilon, Y_{\varepsilon}(s)\right)^{*}\right] d s,} \\
& {[M]_{t} \leq 2 \int_{0}^{t \wedge \tau_{x}^{\varepsilon}}\left\|\sqrt[4]{\varepsilon} \widehat{\Phi}\left(\varepsilon, Y_{\varepsilon}(s)\right)\right\|_{E n d\left(\mathbb{R}^{m}\right)}^{2} d s .}
\end{aligned}
$$

Hence:

$$
\begin{aligned}
& \mathrm{V} \leq P_{x}\left\{Z_{\varepsilon}(t) \geq \varepsilon^{\beta} ; \chi_{1}=0\right\} \\
& \mathrm{V} \leq P\left\{\sup _{[0, t]}\left\|M_{s}\right\| \geq \varepsilon^{\beta}-t C_{3} \delta \varepsilon^{\alpha+\frac{1}{4}} ;[M]_{t} \leq t\left(C_{2} \delta^{2} \varepsilon^{2 \alpha}\right)^{2}\right\} \\
& \mathrm{V} \leq 2 m \exp -\frac{\left(\varepsilon^{\beta}-t C_{3} \delta \varepsilon^{\alpha+\frac{1}{4}}\right)^{2}}{2 m t C_{2}^{2} \delta^{4} \varepsilon^{4 \alpha}} .
\end{aligned}
$$

\section{Estimate of VI}

By Taylor formula there exist a constant $C_{1}>0$ depending only on the function $c$ such that for $\mathrm{x} \in U_{P}$ :

$$
\left|c(x)-c(P)-\sum_{n=1}^{m} \lambda_{n} x_{n}^{2}\right| \leq C_{1}|| x \|_{\mathbb{R}^{m}} \sum_{1}^{m} \lambda_{i} x_{i}^{2}
$$

Then, recalling that $\mathrm{c}(\mathrm{P})=\min c$ and $\mathrm{c}_{\varepsilon}=\frac{c-\min _{V_{m}} c}{\sqrt{\epsilon}}$ for $\mathrm{t}<\tau_{\varepsilon}^{U}$ :

$$
\left(1-\sqrt[4]{\varepsilon} C_{1}\left\|Y_{\varepsilon}(t)\right\|_{\mathbb{R}^{m}}\right) \sum_{1}^{m} \lambda_{i} Y_{\varepsilon, i}(t)^{2} \leq c_{\varepsilon}\left(X_{\epsilon}(t)\right) \leq\left(1+\sqrt[4]{\varepsilon} C_{1}\left\|Y_{\varepsilon}(t)\right\|_{\mathbb{R}^{m}}\right) \sum_{1}^{m} \lambda_{i} Y_{\varepsilon, i}(t)^{2}
$$


For the sake of simplicity let us denote the positive definite quadratic form $\sum_{1}^{m} \lambda_{i} X_{i}^{2}$ by $q(X)$. Given any $\sigma>0$, any $a, b \in \mathbb{R}^{m}$ we have:

$$
(1-\sigma) q(a)+\left(1-\frac{1}{\sigma}\right) q(b) \leq q(a+b) \leq(1+\sigma) q(a)+\left(1+\frac{1}{\sigma}\right) q(b) .
$$

Now:

$$
q\left(Y_{\varepsilon}(t)\right)=q\left(Y_{\varepsilon}(0)+\sqrt{2} W(t)+Z_{\varepsilon}(t)\right) .
$$

Using the inequality (26) taking $a=Y_{\varepsilon}(0)+\sqrt{2} W(t), b=Z_{\varepsilon}(t), \quad \sigma=1$ :

$$
q\left(Y_{\varepsilon}(t)\right) \geq \frac{1}{2} q\left(Y_{\varepsilon}(0)+\sqrt{2} W(t)\right)-q\left(Z_{\varepsilon}(t)\right) .
$$

Hence setting $\mathrm{y}=\frac{x}{\sqrt[4]{\varepsilon}}=Y_{\varepsilon}(0)$, for $\mathrm{y} \in B\left(\delta \varepsilon^{\alpha-\frac{1}{4}}\right)$ :

$$
\begin{gathered}
\mathrm{VI}=E_{y}\left(\left(1-\chi_{1}\right) \chi_{\left(t<\tau_{\epsilon}^{x}\right)} e^{-\int_{0}^{t} c_{\epsilon}\left(\sqrt[4]{\varepsilon} Y_{\epsilon}(s)\right) d s} ; \sup _{\left[0, t \wedge \tau_{\varepsilon}^{x}\right]}\left\|Z_{\varepsilon}(s)\right\|_{\mathbb{R}^{m}}<\varepsilon^{\beta}\right) \\
\mathrm{VI} \leq E_{y}\left(\left(1-\chi_{1}\right) \chi_{\left(t<\tau_{\epsilon}^{x}\right)} \exp -\left(1-\delta \sqrt[4]{\varepsilon} C_{1}\right) \int_{0}^{t} q\left(Y_{\varepsilon}(s)\right) d s ; \sup _{\left[0, t \wedge \tau_{\varepsilon}^{x}\right]}\left\|Z_{\varepsilon}(s)\right\|_{\left.\mathbb{R}^{m}<\varepsilon^{\beta}\right)}\right. \\
\mathrm{VI} \geq E_{y}\left(\left(1-\chi_{1}\right) \chi_{\left(t<\tau_{\epsilon}^{x}\right)} \exp -\left(1+\delta \sqrt[4]{\varepsilon} C_{1}\right) \int_{0}^{t} q\left(Y_{\varepsilon}(s)\right) d s ; \sup _{\left[0, t \wedge \tau_{\varepsilon}^{x}\right]}\left\|Z_{\varepsilon}(s)\right\|_{\mathbb{R}^{m}}<\varepsilon^{\beta}\right)
\end{gathered}
$$

Using the inequality(27):

$$
\begin{aligned}
\mathrm{VI} \leq & E_{y}\left[( 1 - \chi _ { 1 } ) \chi _ { ( t < \tau _ { \epsilon } ^ { x } ) } \operatorname { e x p } ( 1 - \delta \sqrt [ 4 ] { \varepsilon } C _ { 1 } ) \int _ { 0 } ^ { t } q \left(Z_{\varepsilon}(s) d s-\right.\right. \\
& \left.\left(1-\delta \sqrt[4]{\varepsilon} C_{1}\right) \frac{1}{2} \int_{0}^{t} q(y+\sqrt{2} W(t)) d s ; \sup _{\left[0, t \wedge \tau_{\varepsilon}^{x}\right]}\left\|Z_{\varepsilon}(s)\right\|_{\mathbb{R}^{m}}<\varepsilon^{\beta}\right] \\
\mathrm{VI} \leq & C_{4}(\delta, \varepsilon, t) E_{0}\left(\exp -\left(1-\delta \sqrt[4]{\varepsilon} C_{1}\right) \frac{1}{2} \int_{0}^{t} q(y+\sqrt{2} W(s)) d s\right)
\end{aligned}
$$

where:

$$
\begin{aligned}
& C_{4}(\delta, \varepsilon, t)=\exp \left(\left(1-\delta \sqrt[4]{\varepsilon} C_{1}\right) t \sqrt{\varepsilon} \max \left\{q(v) \mid\|v\|_{\mathbb{R}^{m}}<\varepsilon^{\beta}\right\}\right) \\
& \left.C_{4}(\delta, \varepsilon, t)=\exp \left(1-\delta \sqrt[4]{\varepsilon} C_{1}\right) \varepsilon^{\frac{1}{2}+2 \beta} \max \left\{q(v) \mid\|v\|_{\mathbb{R}^{m}}<1\right\}\right) .
\end{aligned}
$$

Note that $C_{4}(\delta, \varepsilon, t) \longrightarrow 1$ as $\varepsilon \rightarrow>0$ (keeping t fixed). Let us estimate the expectation $E_{0}\left(\exp -\left(1-\delta \sqrt[4]{\varepsilon} C_{1}\right) \frac{1}{2} \int_{0}^{t} q(y+W(t)) d s\right)$. For simplicity set $a(\varepsilon)=\left(1-\delta \sqrt[4]{\varepsilon} C_{1}\right)$. Then:

$$
E_{0}\left(\exp -\frac{a(\varepsilon)}{2} \int_{0}^{t} q(y+\sqrt{2} W(t)) d s\right)=\Pi_{i=1}^{m} E_{0}\left(\exp -\frac{a(\varepsilon)}{2} \int_{0}^{t} \lambda_{i}\left(y_{i}+\sqrt{2} W_{i}(s)\right)^{2} d s\right)
$$

where $y=\left(y_{1}, \ldots y_{m}\right)$ and $\mathrm{W}(\mathrm{t})=\left(\mathrm{W}_{1}(\mathrm{t}), \ldots, \mathrm{W}_{m}(\mathrm{t})\right)$.

To find the value of $E_{0}\left(\exp -\frac{a(\varepsilon)}{2} \int_{0}^{t} \lambda_{i}\left(y_{i}+\sqrt{2} W_{i}(s)\right)^{2} d s\right)$ we use the following lemma: 
Lemma 3 Consider $w(s)$ the Brownian motion in $\mathbb{R}$, a positive real number $\lambda>0$ and the function $z_{\mu}^{\lambda}$ :

$$
(x, t) \in \mathbb{R} \times \mathbb{R}^{+} \rightarrow E_{x}\left[e^{-\int_{0}^{t} \lambda\left(\mu w(s)+w(s)^{2}\right) d s}\right]
$$

where $E_{x}$ is the expectation for a process starting at a point $x$. Then $z_{\mu}^{\lambda}$ is a bounded solution of the parabolic equation,

$$
\begin{aligned}
\frac{\partial z}{\partial t} & =\frac{1}{2} \frac{\partial^{2} z}{\partial x^{2}}-\lambda\left(x^{2}+\mu x\right) z, \text { for } x \in \mathbb{R}, t \geq 0 \\
z(0, x) & =1 .
\end{aligned}
$$

For all $x \in \mathbb{R}, t \geq 0$, the solution is given by

$$
z_{\mu}^{\lambda}(x, t)=\frac{1}{\sqrt{\cosh (t \sqrt{2 \lambda})}} \exp \left(-\frac{\sqrt{\lambda} \tanh (t \sqrt{2 \lambda})}{\sqrt{2}}\left(x+\frac{\mu}{2}\right)^{2}+\frac{\lambda \mu^{2} t}{4}\right)
$$

and the value of the expectation at the origin 0 is,

$$
z_{\mu}^{\lambda}(x, t)=\frac{1}{\sqrt{\cosh (t \sqrt{2 \lambda})}} \exp \left(-\frac{\sqrt{\lambda} \tanh (t \sqrt{2 \lambda})}{\sqrt{2}}\left(\frac{\mu}{2}\right)^{2}+\frac{\lambda \mu^{2} t}{4}\right) .
$$

\section{Assuming the lemma we pursue the evaluation of :}

$$
\begin{array}{r}
E_{0}\left(\exp -\frac{a(\varepsilon)}{2} \int_{0}^{t} \lambda_{i}\left(y_{i}+\sqrt{2} W_{i}(s)\right)^{2} d s\right) \\
=e^{-\frac{a(\varepsilon) t \lambda_{i} y_{i}^{2}}{2}} E_{y_{i}}\left(\exp -\frac{a(\varepsilon)}{2} \int_{0}^{t} \lambda_{i}\left[2 \sqrt{2} y_{i} w_{i}(s)+\sqrt{2} W_{i}(s)^{2}\right] d s\right) \\
E_{0}\left(\exp \left[-\frac{a(\varepsilon)}{2} \int_{0}^{t} \lambda_{i}\left(y_{i}+\sqrt{2} W_{i}(s)\right)^{2} d s\right]\right)= \\
\frac{e^{-\frac{a(\varepsilon) t \lambda_{i} y_{i}^{2}}{2}}}{\sqrt{\cosh \left(t \sqrt{2 a(\varepsilon) \lambda_{i}}\right)}} \exp -\left(\frac{\sqrt{a(\varepsilon) \lambda_{i}} \tanh \left(t \sqrt{2 a(\varepsilon) a \lambda_{i}}\right)}{2 \sqrt{2}} y_{i}^{2}+\frac{a(\varepsilon) \lambda_{i} y_{i}^{2} t}{2}\right),
\end{array}
$$

and

$$
E_{0}\left(\exp \left[-\frac{a(\varepsilon)}{2} \int_{0}^{t} \lambda_{i}\left(y_{i}+\sqrt{2} W_{i}(s)\right)^{2} d s\right]\right)=\frac{\exp \left(-\frac{\sqrt{a(\varepsilon) \lambda_{i}} \tanh \left(t \sqrt{2 a(\varepsilon) \lambda_{i}}\right)}{2 \sqrt{2}} y_{i}^{2}\right)}{\sqrt{\cosh \left(t \sqrt{2 a(\varepsilon) \lambda_{i}}\right)}} .
$$

Finally:

$$
\mathrm{VI} \leq \frac{C_{4}(\delta, \varepsilon, t)}{\prod_{i=1}^{m} \sqrt{\cosh \left(t \sqrt{2 a(\varepsilon) \lambda_{i}}\right)}} \exp \left(-\sum_{i=1}^{m} \frac{\sqrt{a(\varepsilon) \lambda_{i}} \tanh \left(t \sqrt{2 a(\varepsilon) \lambda_{i}}\right)}{2 \sqrt{2}} y_{i}^{2}\right) .
$$




\section{End of the proof of Proposition 3}

Now we can wrap up the proof of Proposition 3 For any $\alpha \in] 0, \frac{1}{6}[$, any $\beta \in] 0, \alpha[$, any $\left.\varepsilon \in] 0, \varepsilon_{0}\right]$ where $\varepsilon_{0}<1$ depends only on $\alpha, \beta, \delta$ and any integer $\mathrm{k}$, we get the estimate if $\mathrm{x} \in B_{P}\left(\frac{\delta \varepsilon^{\alpha}}{2}\right):$

$$
\begin{aligned}
\frac{u_{\epsilon}(x)}{\overline{u_{\varepsilon}}} e^{-\mu_{\varepsilon} t} & \leq A(k, \delta) \epsilon^{k}+\frac{C_{4}(\delta, \varepsilon, t)}{\sqcap_{i=1}^{m} \sqrt{\cosh \left(t \sqrt{2 a \lambda_{i}}\right)}} \exp \left(-\sum_{i=1}^{m} \frac{\sqrt{a(\varepsilon) \lambda_{i}} \tanh \left(t \sqrt{2 a(\varepsilon) \lambda_{i}}\right)}{2 \sqrt{2 \varepsilon}} x_{i}^{2}\right) \\
& +2 m\left[\exp -\frac{\left(\varepsilon^{\beta}-t C_{3} \delta \varepsilon^{\alpha}\right)^{2}}{2 m t C_{2}^{2} \delta^{4} \varepsilon^{4 \alpha}}+\exp -\frac{\left.\left[\frac{\delta \varepsilon^{\alpha}}{2}-M_{1} \sqrt{\varepsilon}\right)\right]^{2}}{2 m t M^{2} \sqrt{\varepsilon}}\right]
\end{aligned}
$$

Define the function $w_{\varepsilon}: \mathrm{B}_{P}(\delta) \rightarrow \mathbb{R}_{+}$,

$$
w_{\varepsilon}(y)=\frac{u_{\epsilon}(y \sqrt[4]{\varepsilon})}{\overline{u_{\varepsilon}}}
$$

Then for $\mathrm{y} \in \mathrm{B}_{P}\left(\delta / \varepsilon^{\frac{1}{4}-\alpha}\right)$ :

$$
\begin{aligned}
w_{\varepsilon}(y) e^{-\mu_{\varepsilon} t} & \leq A(k, \delta) \epsilon^{k}+\frac{C_{4}(\delta, \varepsilon, t)}{\prod_{i=1}^{m} \sqrt{\cosh \left(t \sqrt{2 a \lambda_{i}}\right)}} \exp \left(-\sum_{i=1}^{m} \frac{\sqrt{a(\varepsilon) \lambda_{i}} \tanh \left(t \sqrt{2 a(\varepsilon) \lambda_{i}}\right)}{2 \sqrt{2}} y_{i}^{2}\right) \\
& +2 m\left[\exp -\frac{\left(\varepsilon^{\beta}-t C_{3} \delta \varepsilon^{\alpha}\right)^{2}}{2 m t C_{2}^{2} \delta^{4} \varepsilon^{4 \alpha}}+\exp -\frac{\left.\left[\frac{\delta}{2}-t M_{1} \sqrt{\varepsilon}\right)\right]^{2}}{2 m t M^{2} \sqrt{\varepsilon}}\right]
\end{aligned}
$$

We want to estimate $\sup \left\{w_{\varepsilon}(y) \mid \mathrm{y} \in B_{P}\left(\delta / \varepsilon^{\frac{1}{4}}\right)\right\}$.

$$
\begin{aligned}
& \sup \left\{w_{\varepsilon}(y) \mid y \in B_{P}\left(\delta / \varepsilon^{\frac{1}{4}}\right)\right\} \leq \sup \left\{w_{\varepsilon}(y) \mid \mathrm{y} \in B_{P}\left(\delta / \varepsilon^{\frac{1}{4}}\right)-B_{P}\left(\delta / 2 \varepsilon^{\frac{1}{4}-\alpha}\right)\right\} \\
& \left.\sup \left\{w_{\varepsilon}(y) \mid y \in B_{P}\left(\delta / \varepsilon^{\frac{1}{4}}\right)\right\} \leq \sup \left\{w_{\varepsilon}(y) \mid y \in B_{P}\left(\delta / 2 \varepsilon^{\frac{1}{4}-\alpha}\right)\right\}\right)
\end{aligned}
$$

$\sup \left\{w_{\varepsilon}(y) \mid \mathrm{y} \in B_{P}\left(\delta / \varepsilon^{\frac{1}{4}}\right)-B_{P}\left(\delta / 2 \varepsilon^{\frac{1}{4}-\alpha}\right)\right\} \leq \sup \left\{\frac{u_{\varepsilon}(x)}{\bar{u}_{\varepsilon}} \mid \mathrm{x} \in B_{P}(\delta)-B_{P}\left(\delta \varepsilon^{\alpha} / 2\right)\right\}$

Using Appendix 2 as in the evaluation of the boundary integral I, taking $\varepsilon=\varepsilon, \mathrm{b}=0$, $\psi=c-\min _{V} c, \mathrm{c}_{\varepsilon}=\min _{V} \mathrm{c}-\lambda_{\varepsilon}$ we get for all $\mathrm{n} \geq 1$

$$
\sup \left\{\frac{u_{\varepsilon}(x)}{\bar{u}_{\varepsilon}} \mid \mathrm{x} \in B_{P}(\delta)-B_{P}\left(\delta \varepsilon^{\alpha} / 2\right)\right\} \leq C(n) \frac{\varepsilon^{\frac{n}{3}}}{(\min \psi)^{n}}
$$

where $\min \psi$ is the minimum of $\psi$ on $B_{P}(\delta)-B_{P}\left(\delta \varepsilon^{\alpha} / 2\right)$ and $\mathrm{C}(\mathrm{n})$ is a constant depending on the data $g, c$ and on $\mathrm{n}$ but not on $\varepsilon$. Now there exists a constant $\mathrm{Q}>0$, such that for 
all $x \in \mathrm{V}, c(x) \geq \min _{V} c+\mathrm{Qd}_{g}\left(x, C_{\min }\right)^{2}$. Hence min $\psi \geq \frac{Q}{4}\left(\delta \varepsilon^{\alpha}\right)^{2}$. Since $\alpha<\frac{1}{6}$, we see that for any $\mathrm{k} \in \mathbb{N}$, there exists a constant $\gamma(k)>0$, depending on $\delta$, such that:

$$
\sup \left\{\frac{u_{\varepsilon}(x)}{\bar{u}_{\varepsilon}} \mid \mathrm{x} \in B_{P}(\delta)-B_{P}\left(\delta \varepsilon^{\alpha} / 2\right)\right\} \leq \gamma(k) \varepsilon^{k}
$$

Hence:

$$
\sup \left\{w_{\varepsilon}(y) \mid \mathrm{y} \in B_{P}\left(\delta / \varepsilon^{\frac{1}{4}}\right)-B_{P}\left(\delta / 2 \varepsilon^{\frac{1}{4}-\alpha}\right)\right\} \leq \gamma(k) \varepsilon^{k}
$$

Finally for $\mathrm{x} \in B_{P}(\delta)$ :

$$
\begin{aligned}
& e^{-\mu_{\varepsilon} t} \frac{u_{\varepsilon}(x)}{\bar{u}_{\varepsilon}} \leq \frac{C_{4}(\delta, \varepsilon, t)}{\prod_{i=1}^{m} \sqrt{\cosh \left(t \sqrt{2 a(\varepsilon) \lambda_{i}}\right)}} \exp \left(-\sum_{i=1}^{m} \frac{\sqrt{a(\varepsilon) \lambda_{i}} \tanh \left(t \sqrt{2 a(\varepsilon) \lambda_{i}}\right)}{2 \sqrt{2 \varepsilon}} x_{i}^{2}\right)+ \\
& \quad+(A(k, \delta)+\gamma(k)) \epsilon^{k}+2 m\left[\exp -\frac{\left(\varepsilon^{\beta}-t C_{3} \delta \varepsilon^{\alpha}\right)^{2}}{2 m t C_{2}^{2} \delta^{4} \varepsilon^{4 \alpha}}+\exp -\frac{\left.\left[\frac{\delta \varepsilon^{\alpha}}{2}-t M_{1} \sqrt{\varepsilon}\right)\right]^{2}}{2 m t M^{2} \sqrt{\varepsilon}}\right]
\end{aligned}
$$

Equivalently, for all $\mathrm{y} \in B_{P}(\delta / \sqrt[4]{\varepsilon})$ :

$$
\begin{aligned}
& w_{\varepsilon}(y) e^{-\mu_{\varepsilon} t} \leq \frac{C_{4}(\delta, \varepsilon, t)}{\prod_{i=1}^{m} \sqrt{\cosh \left(t \sqrt{2 a(\varepsilon) \lambda_{i}}\right)}} \exp \left(-\sum_{i=1}^{m} \frac{\sqrt{a(\varepsilon) \lambda_{i}} \tanh \left(t \sqrt{2 a(\varepsilon) \lambda_{i}}\right)}{2 \sqrt{2}} y_{i}^{2}\right)+ \\
& +(A(k, \delta)+\gamma(k)) \epsilon^{k}+2 m\left[\exp -\frac{\left(\varepsilon^{\beta}-t C_{3} \delta \varepsilon^{\alpha}\right)^{2}}{2 m t C_{2}^{2} \delta^{4} \varepsilon^{4 \alpha}}+\exp -\frac{\left.\left[\frac{\delta \varepsilon^{\alpha}}{2}-t M_{1} \sqrt{\varepsilon}\right)\right]^{2}}{2 m t M^{2} \sqrt{\varepsilon}}\right]
\end{aligned}
$$

Given a function $\mathrm{f}$ as in the statement of Theorem [2, for any integer $N$ such that sup $\frac{|f(\varepsilon, y)|}{1+|| y||_{\mathbb{R}^{m}}^{N}}<+\infty$, for any integer $k>\frac{m}{8}+N$ inequality(29) implies that

$$
\sup _{] 0, \varepsilon_{0}\right]} \int_{B_{P}(\delta / \sqrt[4]{\varepsilon})}|f(\varepsilon, y)| w(y)^{2} d y<+\infty
$$

This is the statement (i) of Proposition 3.

(ii)Fixing t and choosing an integer $\mathrm{k}>\frac{m}{8}+N$, we have:

$$
\begin{aligned}
& \lim _{\varepsilon->0} \operatorname{vol}\left(B _ { P } ( \delta / \sqrt [ 4 ] { \varepsilon } ) [ 1 + ( \frac { \delta } { \sqrt [ 4 ] { \varepsilon } } ) ^ { N } ] \left[A(k, \delta) \epsilon^{k}+\gamma(k) \epsilon^{k}+2 m \exp -\frac{\left(\varepsilon^{\beta}-t C_{3} \delta \varepsilon^{\alpha}\right)^{2}}{2 m t C_{2}^{2} \delta^{4} \varepsilon^{4 \alpha}}\right.\right. \\
& \left.+2 m \exp -\frac{\left.\left[\frac{\delta \varepsilon^{\alpha}}{2}-t M_{1} \sqrt{\varepsilon}\right)\right]^{2}}{2 m t M^{2} \sqrt{\varepsilon}}\right]^{2} e^{2 t \mu_{\varepsilon}}=0
\end{aligned}
$$

Hence there exists an $\varepsilon_{1}(\eta)$ such that for all $\left.\left.\varepsilon \in\right] 0, \varepsilon_{1}(\eta)\right]$ : 
1. $\left(\varepsilon^{\beta}-t C_{3} \delta \varepsilon^{\alpha}\right)^{2} \geq \frac{\varepsilon^{2 \beta}}{2}$,

2. $\left.\left[\frac{\delta \varepsilon^{\alpha}}{2}-t M_{1} \sqrt{\varepsilon}\right)\right]^{2} \geq \frac{\delta^{2} \varepsilon^{2 \alpha}}{16}$,

3. a $(\varepsilon)=\left(1-\delta \sqrt[4]{\varepsilon} C_{1}\right) \geq \frac{1}{2}$, As a consequence there exists an $\varepsilon(\eta, t, k), 0 \leq \varepsilon(\eta, t, k) \leq$ $\varepsilon_{1}(\eta)$, such that for $\left.\left.\varepsilon \in\right] 0, \varepsilon(\eta, t, k)\right]$

4. $\operatorname{vol}\left(B_{P}(\delta / \sqrt[4]{\varepsilon})\left((A(k, \delta)+\gamma(k)) \epsilon^{k}+2 m\left[\exp -\frac{\left(\varepsilon^{\beta}-t C_{3} \delta \varepsilon^{\alpha}\right)^{2}}{2 m t C_{2}^{2} \delta^{4} \varepsilon^{4 \alpha}}+\exp -\frac{\left.\left[\frac{\delta \varepsilon^{\alpha}}{2}-t M_{1} \sqrt{\varepsilon}\right)\right]^{2}}{2 m t M^{2} \sqrt{\varepsilon}}\right]\right)^{2} e^{2 t \mu_{\varepsilon}} \leq\right.$ $\frac{\eta}{4}$.

Then we can choose a ball $\mathrm{B}^{m}(\mathrm{P}, \mathrm{R})$ such that for all $\left.\left.\varepsilon \in\right] 0, \varepsilon(\eta, t, k)\right]$ :

$\int_{\mathbb{R}^{m}-B^{m}(0, R)}\left(\frac{C_{4}(\delta, \varepsilon, t)}{\prod_{i=1}^{m} \sqrt{\cosh \left(t \sqrt{2 a(\varepsilon) \lambda_{i}}\right)}} \exp \left(-\sum_{i=1}^{m} \frac{\sqrt{a(\varepsilon) \lambda_{i}} \tanh \left(t \sqrt{2 a(\varepsilon) \lambda_{i}}\right)}{2 \sqrt{2}} y_{i}^{2}\right)\right)^{2} e^{2 t \mu_{\varepsilon}} d y \leq \frac{\eta}{4}$

These two inequalities prove the statement (ii) of Proposition 3 .

\subsubsection{Proof of Lemma 3}

The fact that the function $z_{\mu}^{\lambda}$ satisfies equation (28) is a consequence of Feynman-Kac formula ([29]). Making the change of coordinate $x \rightarrow x-\mu / 2$, the problem becomes:

$$
\begin{gathered}
\frac{\partial z}{\partial t}=\frac{1}{2} \frac{\partial^{2} z}{\partial x^{2}}-\lambda\left(x^{2}-\frac{\mu^{2}}{4}\right) z \\
z(0, x)=1
\end{gathered}
$$

To solve this equation, by the uniqueness of solutions for the Cauchy problem for the parabolic equation ([24]), it is enough to find a solution of the type $e^{-\phi(t) x^{2}+\psi(t)}$. A simple computation leads to the two coupled equations:

$$
\begin{aligned}
\dot{\phi}+2 \phi^{2} & =\lambda \\
\dot{\psi}+\phi & =\frac{\lambda \mu^{2}}{4}
\end{aligned}
$$

which can be solved easily using the initial condition at time zero,

$$
\begin{aligned}
& \phi(t)=\sqrt{\frac{\lambda}{2}} \tanh (t \sqrt{2 \lambda}) \\
& \psi(t)=-\frac{1}{2} \log \cosh (t \sqrt{2 \lambda})+\frac{\lambda \mu^{2} t}{4}
\end{aligned}
$$

so we obtain the expression

$$
z_{\mu}^{\lambda}(x, t)=\frac{1}{\sqrt{\cosh (t \sqrt{2 \lambda})}} \exp \left(-\frac{\sqrt{\lambda} \tanh (t \sqrt{2 \lambda})}{\sqrt{2}}\left(x+\frac{\mu}{2}\right)^{2}+\frac{\lambda \mu^{2} t}{4}\right) .
$$




\subsection{Proof of the main theorem}

We use the notations of the preceding sections. Because $x=\left(x_{1}, . ., x_{m}\right)$ is a normal coordinate system centered at $P \in C_{\text {min }}$, the eigenfunction $w_{\varepsilon}$ satisfies the following renormalized equation, derived from equation (21), in the blown up coordinates, $y=\sqrt[4]{\epsilon} x$ :

$$
\Delta_{g_{\epsilon}} w_{\epsilon}+\frac{c(\sqrt[4]{\varepsilon} x)-\min _{V} c}{\sqrt[2]{\varepsilon}} w_{\epsilon}=\frac{\lambda_{\epsilon}-\min _{V} c}{\sqrt[2]{\varepsilon}} w_{\epsilon}, \text { in } \frac{1}{\sqrt[4]{\varepsilon}} \Omega=\frac{1}{\sqrt[4]{\varepsilon}} x_{1} \times \ldots \times x_{m}(U)
$$

where $g_{\epsilon}$ is the metric rescaled by $\epsilon$, converging to the Euclidean metric uniformly on every compact set of $\mathbb{R}^{m}$ in the $\mathrm{C}^{\infty}$ topology:

$$
\begin{gathered}
g_{\varepsilon, i j}(y)=g_{i j}(y \sqrt[4]{\varepsilon}), \text { on } \frac{1}{\sqrt[4]{\varepsilon}} \Omega \\
\Delta_{g_{\epsilon}} w=-\sum_{i, j=1}^{m} g^{i j}(y \sqrt[4]{\varepsilon}) \frac{\partial^{2} w(y)}{\partial y^{i} \partial^{j} y}+\sum_{i, j=1}^{m} \sqrt[4]{\varepsilon} g^{i j}(y \sqrt[4]{\varepsilon}) \Gamma_{i j}^{k}(y \sqrt[4]{\varepsilon})\left(\frac{\partial w(y)}{\partial y^{k}}\right)
\end{gathered}
$$

Remark that all coefficients of the partial differential equation (301) are bounded. In particular this term is bounded with $\epsilon$. By Lemma 2 2 the quantity $\frac{\lambda_{\epsilon}-\min _{V} c}{\epsilon^{1 / 2}}$ is bounded and nonnegative.

By the classical theory of elliptic partial differential equations, since $w_{\epsilon}$ is bounded by 1 for all $\varepsilon$, any sequence $\left\{w_{\epsilon_{n}}\right\}$ with $\varepsilon_{n}$ tending to 0 contains a subsequence, still denoted by $\left\{w_{\epsilon_{n}}\right\}$ for simplicity, which converges to a solution of the following elliptic equation in the $\mathrm{C}^{\infty}$ topology:

$$
\Delta_{E} w+\sum_{n=1}^{m} \lambda_{n} y_{n}^{2} w=\lambda w \text { on } \mathbb{R}^{m}
$$

where:

$$
\lambda=\lim _{n \rightarrow \infty} \frac{\lambda_{\epsilon_{n}}-\min _{V} c}{\epsilon_{n}^{1 / 2}}
$$

and for simplicity:

$$
\lambda_{n}=\lambda_{n}(P)
$$

For every compact $K \in \mathbb{R}^{m}$ :

$$
\int_{K} w(y)^{2} d y=\lim _{n-->\infty} \int_{K} w_{\varepsilon_{n}}(y)^{2} d y \leq \sup _{] 0, \varepsilon_{0}\right]} \int_{B_{P}(\delta / \sqrt[4]{\varepsilon})} w_{\varepsilon}(y)^{2} d y<+\infty
$$

Hence $\mathrm{w} \in L^{2}\left(\mathbb{R}^{m}\right)$ and $\|\mathrm{w}\|_{L^{2}\left(\mathbb{R}^{m}\right)} \leq \lim _{n-->\infty}\left\|\mathrm{w}_{\varepsilon_{n}}\right\|_{L^{2}\left(\mathbb{R}^{m}\right)}$. In fact $\mathrm{w}=\lim _{n-->\infty} \mathrm{w}_{\varepsilon_{n}}$ in $L^{2}\left(\mathbb{R}^{m}\right)$. To see this assume that $\inf _{n}\left\|w_{\varepsilon_{n}}-w\right\|_{L^{2}\left(\mathbb{R}^{m}\right)} \geq \xi>0$. By (ii) of Proposition 3 and the fact that w $\in L^{2}\left(\mathbb{R}^{m}\right)$, we can find a compact $\mathrm{K}$ and an integer $N_{1}$ such that $\int_{\mathbb{R}^{m}-K} w_{\varepsilon_{n}}(y)^{2} d y \leq$ $\left(\frac{\xi}{4}\right)^{2}$ if $n \geq N_{1}$, and $\int_{\mathbb{R}^{m}-K} w(y)^{2} d y \leq\left(\frac{\xi}{4}\right)^{2}$. 
Now there exists an integer $N_{2}$ such that $\int_{K}\left(w_{\varepsilon_{n}}(y)-w(y)\right)^{2} d y \leq\left(\frac{\xi}{4}\right)^{2}$ if $\mathrm{n} \geq N_{2}$. Then for $\mathrm{n} \geq \max \left(N_{1}, N_{2}\right),\left\|w_{\varepsilon_{n}}-w\right\|_{L^{2}\left(\mathbb{R}^{m}\right)}^{2} \leq 3\left(\frac{\xi}{4}\right)^{2}$. This contradicts the assumption inf $\left\|w_{\varepsilon_{n}}-w\right\|_{L^{2}\left(\mathbb{R}^{m}\right)} \geq \xi>0$.

Since $w$ is not zero, equation (31) is an eigenvalue problem. To determine the solutions to this problem, consider the unbounded operator $L: D \longrightarrow L^{2}\left(\mathbb{R}^{m}\right)$,

$$
L w=\Delta_{E} w+\sum_{i=1}^{m} \lambda_{i} y_{i}^{2} w
$$

and

$$
D=\left\{u \in L^{2}\left(\mathbb{R}^{m}\right) \mid u \in H^{2}\left(\mathbb{R}^{m}\right),\left[\sum_{n=1}^{m} \lambda_{n} y_{n}^{2}\right] u \in L^{2}\left(\mathbb{R}^{m}\right)\right\},
$$

$L$ is a self adjoint operator, the spectrum of which we want to compute.

Let us introduce the one dimensional unbounded operators $L_{n}: D_{1} \longrightarrow L^{2}(\mathbb{R})$ where $D_{1}=\left\{u \in L^{2}(\mathbb{R}) \mid u \in H^{2}(\mathbb{R}), u x^{2} \in L^{2}(\mathbb{R})\right\} . \quad L_{n} u=-\frac{d^{2} u}{d y^{2}}+\lambda_{n} y^{2} u, L_{n}$ is a self adjoint operator, the Hermite operator. It is well known that $L^{2}\left(\mathbb{R}^{m}\right)$ can be identified to the m-fold projective tensor product $L^{2}(\mathbb{R}) \widehat{\otimes} L^{2}(\mathbb{R}) \widehat{\otimes} \ldots, \widehat{\otimes} L^{2}(\mathbb{R})$. Then $\mathrm{L}$ is the self adjoint extension of the operator $\sum_{n=1}^{m} L_{n}: D_{1} \otimes \ldots \otimes D_{1} \longrightarrow L^{2}\left(\mathbb{R}^{m}\right)$.

$$
\left(\sum_{n=1}^{m} L_{n}\right)\left(u_{1}\left(y_{1}\right) \otimes \ldots \otimes u_{m}\left(y_{m}\right)\right)=\sum_{n=1}^{m} u_{1}\left(y_{1}\right) \otimes \cdot L_{n}\left(u_{n}\right)\left(y_{n}\right) \cdot \otimes u_{m}\left(y_{m}\right)
$$

¿From this it follows that the spectrum $\sigma(L)$ of $\mathrm{L}$ is given by the formula:

$$
\sigma(L)=\overline{\sigma\left(L_{1}\right)+\ldots+\sigma\left(L_{m}\right)}
$$

where $\overline{\sigma\left(L_{1}\right)+\ldots+\sigma\left(L_{m}\right)}$ is the closure of the $\operatorname{set}\left\{\mu_{1}+\ldots+\mu_{m} \mid \mu_{k} \in \sigma\left(L_{k}\right), 1 \leq k \leq m\right\}$.

In the present case:

$$
\sigma\left(L_{n}\right)=\left\{(2 k+1) \sqrt{\lambda_{n}} \mid \mathrm{k} \in \mathbb{Z}^{+}\right\}
$$

The eigenfunction (up to multiplication by a scalar) corresponding to the eigenvalue $(2 k+1) \sqrt{\lambda_{n}}$ is the function :

$$
e^{\frac{-\sqrt{\lambda_{n}} y^{2}}{2}} H_{k}\left(y \sqrt[4]{\lambda_{n}}\right)
$$

where $\mathrm{H}_{n}$ is the $\mathrm{k}^{\text {th }}$ Hermite polynomial.

$$
H_{k}(x)=e^{x^{2}} \frac{d^{k}}{d x^{k}} e^{-x^{2}}
$$

Hence the lowest eigenvalue of the operator $L$ is $\sum_{n=1}^{m} \sqrt{\lambda_{n}}$. An associated eigenfunction of $L$ is: $\prod_{n=1}^{m} \exp \left(-\frac{\sqrt{\lambda_{n}} y_{n}^{2}}{2}\right)$. Up to multiplication by a scalar this is the only eigenfunction of $L$ associated to $\sum_{n=1}^{m} \sqrt{\lambda_{n}}$ and it is strictly of one sign. This follows from Friedrichs' theorem and the fact that $\mathrm{L}$ is a positive self adjoint operator (see [27] vol.4 p.207, Thm. XIII.48). By Proposition 2 : 


$$
\lambda \leq \Lambda
$$

Hence we get:

$$
\lambda=\Lambda
$$

and:

$$
w(x)=\prod_{n=1}^{m} \exp \left(-\frac{\sqrt{\lambda_{n}} x_{n}^{2}}{2}\right)=\exp -\sum_{n=1}^{m} \frac{\sqrt{\lambda_{n}} x_{n}^{2}}{2}
$$

Definition $3 C_{\min \min }$ is the subset $\left\{C \mid P \in C_{\min }, \sum_{n=1}^{m} \sqrt{\lambda_{n}(P)}=\Lambda\right\}$.

The following theorem amplifies theorem 1 and sums up all our results in the self adjoint case.

\section{Theorem 3 Distribution of limit measures.}

(i) Using the notations of Lemma Q $\sup _{q \in \mathcal{M}_{\varepsilon}} d_{g}^{2}\left(q, C_{\text {min min }}\right) \leq A \epsilon^{1 / 2}$

(ii)Let $S$ be the weak limit set of the measures $u_{\epsilon}^{2} d v o l_{g} / \int_{V} u_{\varepsilon}^{2} d v o l_{g}$ as $\varepsilon$ goes to zero. Then:

$$
S=\left\{\mu \in M(V) \mid \mu=\sum\left\{\gamma_{P} \delta_{P} \mid P \in C_{\min \min }\right\}\right.
$$

(iii)For any sequence $\left\{u_{\varepsilon_{n}}\right\}$ such that the measures $u_{\varepsilon_{n}}^{2}$ vol $_{g} / \int_{V} u_{\varepsilon_{n}}^{2}$ dvol $l_{g}$ converge weakly to $\mu$, the concentration coefficient $\gamma_{P}$, due to the normalization condition in the $L^{2}$ space, is given

$$
\gamma_{P}=(2 \pi)^{\frac{m}{2}} \frac{f_{P}^{2}}{K} \prod_{k=1}^{m} \lambda_{k}^{-1 / 4}(P)
$$

where

$$
f_{P}=\lim _{n \rightarrow \infty} \frac{\sup _{B_{P_{k}}(\delta)} u_{\varepsilon_{n}}}{\sup _{V_{m}} u_{\varepsilon_{n}}}
$$

and

$$
K=(2 \pi)^{\frac{m}{2}} \sum_{P \in C_{\min } \min } f_{P}^{2} \prod_{k=1}^{m} \lambda_{k}^{-1 / 4}(P)
$$

(iii)If $P$ is a limit point of the sets of maximum points of the $u_{\varepsilon_{n}}$ then $f_{P}=1$.

(iv) There is always a $\bar{P}$ such that $f_{P}=1$

Proof. We shall use the previous notations. Note that:

$$
\int_{V} u_{\epsilon}^{2} d v o l_{g}=\sum_{P \in C_{\min }} \int_{B_{P}(\delta)} u_{\epsilon}^{2} d v o l_{g}+\int_{V-C_{\min }(\delta)} u_{\epsilon}^{2} d v o l_{g}
$$

where

$$
C_{\min }(\delta)=\cup_{P \in C_{\min }} B_{P}(\delta)
$$


¿From now on we normalize the $\mathrm{u}_{\varepsilon}$ requiring that $\int_{V} u_{\epsilon}^{2} d v o l_{g}=1$. Let $\left\{\mathrm{u}_{\varepsilon_{n}} \mid\right\}$ be any sequence such that the measures $\mathrm{u}_{\varepsilon_{n}}^{2} v o l_{g} / \int_{V \mathrm{u} \varepsilon_{n}}^{2} d v o l_{g}$ converge weakly to $\mu \in \mathrm{M}(\mathrm{V})$. Equation 10 implies that:

$$
\lim _{\varepsilon->0} \int_{V-C_{\min }(\delta)} u_{\epsilon_{n}}^{2} d v o l_{g}=0
$$

Hence:

$$
\lim _{\varepsilon \rightarrow>0} \sum_{P \in C_{\min }} \int_{B_{P}(\delta)} u_{\epsilon_{n}}^{2} d v o l_{g}=1
$$

For any $\mathrm{P} \in C_{\min }$ :

$$
\int_{B_{P}(\delta)} u_{\epsilon}^{2} d v o l_{g}=\bar{u}_{\epsilon}^{2} \epsilon_{n}^{m / 4} \int_{B_{P}\left(\delta / \sqrt[4]{\varepsilon_{n}}\right)} w_{P, \epsilon_{n}}^{2} d v o l_{g_{\varepsilon_{n}}}
$$

where $w_{P, \epsilon}$ is the restriction of the function $\frac{u_{\epsilon}(y \sqrt[4]{\varepsilon})}{\bar{u}_{\varepsilon}}$ to $\mathrm{B}_{P}(\delta)$ extended by 0 outside $\mathrm{B}_{P}(\delta)$.

Any sequence of $\epsilon^{\prime}$ s converging to zero contains a subsequence $\left(\epsilon_{n}\right)$ such that for any $\mathrm{P}$ in $C_{\min }, \int_{B_{P}(\delta)} u_{\epsilon_{n}}^{2} d v o l_{g}$ converges as $\mathrm{n}$ goes to $\infty$ and $w_{P, \epsilon_{n}}$ converges to $w_{P}(x)=$ $f_{P} \exp \left(-\sum_{n=1}^{m} \frac{\lambda_{n}(P) x_{n}^{2}}{2}\right)$ by Theorem 2. Also by the same theorem $\mathrm{f}_{P}=1$ for at least one P. Hence the sequence $\left\{\bar{u}_{\epsilon_{n}}^{2} \epsilon_{n}^{m / 4} \mid n \in \mathbb{N}\right\}$ will converge to a constant $\mathrm{K}>0$. We claim that:

$$
\lim _{\varepsilon \rightarrow>0} \int_{B_{P}(\delta / \sqrt[4]{\varepsilon})} w_{P, \epsilon_{n}}^{2} d v o l_{g_{\varepsilon n}}=\int_{\mathbb{R}^{m}} w_{P}(y)^{2} d y=f_{P}^{2} \frac{(2 \pi)^{\frac{m}{2}}}{\sqrt[4]{\prod_{k=1}^{m} \lambda_{k}(P)}}
$$

To see this note that:

$$
\int_{B_{P}(\delta / \sqrt[4]{\varepsilon})} w_{P, \epsilon_{n}}^{2} d \operatorname{vol}_{g_{\varepsilon_{n}}}=\int_{B_{P}(\delta / \sqrt[4]{\varepsilon})} w_{P, \epsilon_{n}}^{2}(y) \sqrt{\operatorname{det}\left(g_{\varepsilon_{n}}\right)} d y .
$$

Using Morse's lemma on $U_{P}$ :

$$
\begin{gathered}
g_{i j}(x)=\delta_{i j}+\sum_{k, l=1}^{m} G_{i j k l}(x) x_{k} x_{l}, \\
g_{\varepsilon i j}(y)=g_{i j}(y \sqrt[4]{\varepsilon})=\delta_{i j}+\sqrt{\varepsilon} \sum_{k, l=1}^{m} G_{i j k l}(y \sqrt[4]{\varepsilon}) y_{k} y_{l} .
\end{gathered}
$$

Hence:

$$
\sqrt{\operatorname{det}\left(g_{\varepsilon i j}\right)(y)}=1+\sqrt{\varepsilon} G(\varepsilon, y)
$$

where:

$$
|G(\varepsilon, y)| \leq C(M)\left(1+\|y\|_{\mathbb{R}^{m}}^{2 m}\right)
$$

$\mathrm{C}(\mathrm{M})$ is a constant depending only on $\sup \left\{\mid G_{i j k l}(x) \| 1 \leq i, j, k, l \leq m, \mathrm{x} \in B_{p}(\delta)\right\}$.

$\int_{B_{P}\left(\delta / \sqrt[4]{\varepsilon_{n}}\right)} w_{P, \epsilon_{n}}^{2}(y) \sqrt{\operatorname{det}\left(\left(g_{\varepsilon_{n}}\right)_{i j}\right)} d y=\int_{B_{P}\left(\delta / \sqrt[4]{\varepsilon_{n}}\right)} w_{P, \epsilon}^{2}(y) d y+\sqrt{\varepsilon} \int_{B_{P}\left(\delta / \sqrt[4]{\varepsilon_{n}}\right)} w_{P, \epsilon_{n}}^{2}(y) G\left(\varepsilon_{n}, y\right) d y$ 
Proposition 3 (i) implies that:

$$
\lim _{n \rightarrow>\infty} \sqrt{\varepsilon_{n}} \int_{B_{P}\left(\delta / \sqrt[4]{\varepsilon_{n}}\right)} w_{P, \epsilon_{n}}^{2}(y) G\left(\varepsilon_{n}, y\right) d y=0
$$

and:

$$
\lim _{n \rightarrow>\infty} \int_{B_{P}\left(\delta / \sqrt[4]{\varepsilon_{n}}\right)} w_{P, \epsilon_{n}}^{2}(y) d y=f_{P}^{2} \int_{\mathbb{R}^{m}} \prod_{k=1}^{m} \exp \left(-\frac{\sqrt{\lambda_{k}(P)} y_{k}^{2}}{2}\right) d y
$$

Hence:

$$
\begin{gathered}
\lim _{n \rightarrow>\infty} \int_{B_{P}\left(\delta / \sqrt[4]{\varepsilon_{n}}\right)} w_{P, \epsilon_{n}}^{2} d v o l_{g_{\varepsilon}}=f_{P}^{2} \frac{(2 \pi)^{\frac{m}{2}}}{\sqrt[4]{\prod_{k=1}^{m} \lambda_{k}(P)}} \\
\lim _{n \rightarrow>\infty} \sum_{P \in C_{\min }} \int_{B_{P}(\delta)} u_{\epsilon_{n}}^{2} d v o l_{g}=1
\end{gathered}
$$

We have then the following alternative for any $P \in C_{\min }$ :

$$
\lim _{n->\infty} \int_{B_{P}(\delta)} u_{\epsilon_{n}}^{2} d v o l_{g}=\left\{\begin{array}{c}
0, \text { if } P \notin \mathrm{C}_{\min \min } \\
\frac{f_{P}^{2}}{K} \frac{(2 \pi)^{\frac{m}{2}}}{\prod_{n=1}^{m} \sqrt[4]{\lambda_{n}(P)}}, \text { if } P \in \mathrm{C}_{\min \min }
\end{array}\right.
$$

Because $\lim _{\varepsilon->0} \sum_{P \in C_{\min }} \int_{B_{P}(\delta)} u_{\epsilon}^{2} d v o l_{g}=1$

$$
K=\sum_{P \in \mathcal{C}_{\min } \min } \frac{f_{P}^{2}(2 \pi)^{\frac{m}{2}}}{\prod_{k=1}^{m} \sqrt[4]{\lambda_{k}(P)}}
$$

Finally Lemma2 implies (iv).

Remark 1: As we shall see in the next section, the Topological Pressure gives enough information to determine where the concentration process occurs. This information is contained in the quantity $c_{0}$ only. But what we actually found is that a second quantity $c_{1}$ which carries some second order information about the potential, narrows down the possible concentration set.

Remark 2: When c is not a Morse function, it is an open problem to compute all the limit and the values of the coefficients.

Remark 3: We insist that not all minimum points are necessarily charged, except when the equations admit some symmetries. It is an open problem to prove that indeed the minimum points of the potential c where the Topological Pressure achieves its minimum are all charged.

Remark 4: The main theorem is useful to interpret the dynamics of a particle on a compact manifold moving under the influence of small random noise. Indeed, as $\epsilon$ goes to zero, the density probability of the particle satisfies the Fokker-Plank equation:

$$
\frac{\partial p}{\partial t}=-\epsilon \Delta p-c p
$$


where $\mathrm{c}$ is a killing term [15]. $p$ can be expanded in a series of eigenfunctions, $\phi_{\epsilon}^{i}$ associated to the eigenvalues $\lambda_{i}$,

$$
p(t, x, y)=\sum_{i} e^{-\lambda_{i} t} \phi_{\epsilon}^{i}(x) \phi_{\epsilon}^{i}(y)
$$

The previous analysis proves that the particle will most likely be found near some specific minimal points of $c$. In general, it is well known by the standard Exit problem (see [29]) that a particle subjected to an attractive field can escape outside the domain of attraction, under the influence of small random noise.

But in the case of a compact manifold, they are several bassins of attraction. The particle wanders in and out of these bassins. But, the particle will most likely be found in the neighborhood of a point of $C_{\min }$, where the topological pressure is achieved.

Corollary 1 Let $W \subset V$ an open subset. Assume that $\partial W \cap C_{\min }$ is empty. If for a sequence $\epsilon_{n}$ tending to zero, $\lim _{n \rightarrow \infty}\left[\int_{W} u_{\epsilon_{n}}^{2} d v o l_{g} / \int_{V} u_{\epsilon_{n}}^{2} d v o l_{g}\right]>0$, then $\lim _{n \rightarrow \infty} \sup _{W} u_{\epsilon_{n}}=$ $+\infty$.

Proof. After taking a subsequence if need be, we can assume that the measures $u_{\epsilon_{n}}^{2} d v o l_{g} / \int_{V} u_{\epsilon_{n}}^{2} d v o l_{g}$ converges weakly to a measure $\sum_{P \in C_{1}} a(P) \delta_{P}, \mathrm{C}_{1}$ subset of $\mathrm{C}_{\min \min }, a(P)>0$ for all $\mathrm{P} \in C_{1}$

$$
\sum_{P \in C_{1}} a(P)=1 . \lim _{n \rightarrow \infty}\left[\int_{W} u_{\epsilon_{n}}^{2} d v o l_{g} / \int_{V} u_{\epsilon_{n}}^{2} d v o l_{g}\right]=\sum_{P \in W \cap C_{1}} a(P) .
$$

Hence $\mathrm{W} \cap C_{1}$ is not empty. Say $\mathrm{Q} \in \mathrm{W} \cap C_{1}$. Using the notation of Theorem 3

$$
\begin{gathered}
f_{Q}^{2}=a(Q) \frac{\sqrt[4]{\prod_{k=1}^{m} \lambda_{k}(Q)}}{(2 \pi)^{\frac{m}{2}}}>0 . \\
\lim _{n \rightarrow \infty} \inf \frac{\sup _{W} u_{\epsilon_{n}}}{\sup _{V} u_{\epsilon_{n}}} \geq \lim _{n \rightarrow \infty} \frac{\sup _{Q}(\delta)}{\sup _{\epsilon_{n}} u_{\epsilon_{n}}}=f_{Q}>0 .
\end{gathered}
$$

Lemma 1 ends the proof.

\subsection{The set of limit measures}

In the previous section, we have proved that the limit measures are concentrated on the set $C_{\text {min min }}$. However we did not determine the concentration coefficients completely. Indeed let $\left\{u_{\epsilon_{n}} \mid n \in \mathbb{N}\right\}$ a converging sequence with $\varepsilon_{n}->0$, If we set $v_{\epsilon_{n}}=\frac{u_{\epsilon_{n}}}{\bar{u}_{\epsilon_{n}}}$, then for each point $P \in C_{\min \min }, \lim _{n->\infty} v_{\epsilon_{n}}(P)=\alpha(P) \in[0,1]$. The concentration coefficients are given by

$$
\gamma_{P}=\frac{\alpha^{2}(P) \prod_{n=1}^{m} \lambda_{n}(P)^{\frac{-1}{4}}}{\sum_{R \in C_{\min \min }} \alpha^{2}(R) \prod_{n=1}^{m} \lambda_{n}(R)^{\frac{-1}{4}}}
$$


At least one of the $\alpha(P)$ is equal to one. At present time, we do not know if the $\alpha(P)$ are independent of the sequence $\left\{u_{\epsilon_{n}} \mid n \in \mathbb{N}\right\}$. The modulating factors $\alpha(P)$, defined on the set $C_{\min \text { min }}$, depend only on the ratio of the relative maximum to the global maximum of the eigenfunction. Given a function $\beta: C_{\min \min } \longrightarrow[0,1]$, is it possible to find a converging sequence $\left\{u_{\epsilon_{n}} \mid n \in \mathbb{N}\right\}$ such that $\alpha(P)=\beta(P)$, for $P \in C_{\min \min }$ ? Or is the function $\alpha$ unique? So far to evaluate the concentration coefficients only the potential $c$ was needed. We shall see in the rest of this section that actually only a subset of $C_{\text {minmin }}$ can be charged. The rest of this section is devoted to study the influence of the Riemannian structure on this selection process.

We will use the following definitions:

Definition 4 A point $P \in C_{\text {minmin }}$ is called a maximally charged point if

$$
\limsup _{(\epsilon, Q) \rightarrow(0, P)} \frac{u_{\epsilon}(Q)}{\max _{V_{m}} u_{\epsilon}}=1
$$

Definition 5 A point $P \in C_{\text {minmin }}$ is called a charged point if

$$
\limsup _{(\epsilon, Q) \rightarrow(0, P)} \frac{u_{\epsilon}(Q)}{\max _{V_{m}} u_{\epsilon}}>0
$$

\section{Remark.}

At this stage we do not know if indeed the analysis can be pushed further to prove that whether the limit measure is unique or not. For example can one find a double well asymmetric potential such that sequence of the first eigenfunctions will concentrate at only one of the two minimum points and another sequence only at the other?

\subsection{Expansion of the eigenfunction}

Recall the blown-up function:

$$
w_{P, \varepsilon}(x)=\frac{u_{\varepsilon}(x / \sqrt[4]{\varepsilon})}{\overline{u_{\varepsilon}}}
$$

By Theorem 2 (i), $w_{P, \epsilon}$ converges to $\mathrm{w}_{P}$ in $\mathrm{L}^{2}\left(\mathbb{R}^{m}\right)$ when $\varepsilon$ goes to 0 .

The divided difference

$$
w_{1, \varepsilon}=\frac{w_{P, \varepsilon}-w_{P}}{\sqrt[4]{\varepsilon}}
$$

as $\varepsilon$ goes to 0 , converges to $w_{1}$ satisfying the equation:

$$
L_{P} w_{1}+\sum_{1 \leq i \leq j \leq k \leq m} c_{i j k} y_{i} y_{j} y_{k} w_{P}=0
$$


where

$$
L_{P}=\Delta_{E}+\left[\sum_{k=1}^{m} \sqrt{\lambda_{k}(P)} y_{k}^{2}-\Lambda\right] I d
$$

and:

$$
c-\min c=\sum_{k=1}^{m} \lambda_{k}(P) x_{k}^{2}+\sum_{1 \leq i \leq j \leq k \leq m} c_{i j k} x_{i} x_{j} x_{k}+\sum_{1 \leq i \leq j \leq k \leq l \leq m}^{m} c_{i j k l}(x) x_{i} x_{j} x_{k} x_{l}
$$

where the $c_{i j k}$ are constants and the $c_{i j k l}, C^{\infty}$ functions on $U_{P}$. The proof of the convergence of the sequence $w_{1, \varepsilon}$ results from various estimates that can be obtained on the sequence and its derivatives, which can be found in [12. But a rough idea of the convergence of the sequence results from the WKB expansion. The estimates are based on the exponential decay at infinity. Those estimates are not used in the remaing parts of the article.

\subsubsection{Hermite functions}

We need Hermite functions. For $\underline{n} \in \mathbb{Z}_{+}^{m}$ define

$$
H_{\underline{n}}\left(x_{1}, \ldots, x_{m}\right)=\prod_{j=1}^{m} h_{n_{j}}\left(x_{j} \sqrt[4]{\lambda_{j}(P)}\right),
$$

where for $n \in \mathbb{Z}_{+}$

$$
h_{n}(x)=e^{\frac{x^{2}}{2}} \frac{d^{n}}{d x^{n}} e^{-x^{2}}
$$

Note that

$$
L_{P} H_{\underline{n}}=2<\underline{n}, \sqrt{\lambda(P)}>H_{\underline{n}}
$$

where $\sqrt{\lambda(P)}=\left(\sqrt{\lambda_{1}}(P), . ., \sqrt{\lambda_{n}}(P)\right)$. It is well known and easy to check that

$$
\int_{\mathbb{R}^{m}} H_{\underline{p}} H_{\underline{q}} d y=0
$$

if $\underline{p}, \underline{q} \in \mathbb{Z}_{+}^{m}$ and $\underline{p} \neq \underline{q}$.

$$
\int_{\mathbb{R}^{m}} H_{\underline{p}}^{2} d y=\frac{\pi^{\frac{m}{2}} \underline{\underline{p} ! 2} \underline{\underline{p}}^{\mid} \mid}{\sqrt[4]{\lambda_{1}(P) . . \lambda_{n}(P)}}
$$

where $|\underline{p}|=\sum_{n=1}^{m} p_{n}, \underline{p !}=\prod_{n=1}^{m} p_{n} !$

In particular: $\mathrm{H}_{i j k}=-8 \sqrt{\lambda_{i} \lambda_{j} \lambda_{k}} y_{i} y_{j} y_{k}$, for $1 \leq i<j<k \leq m, \mathrm{H}_{i i j}=-8 \lambda_{i} \sqrt{\lambda_{j}} y_{i}^{2} y_{j}+$ $4 \sqrt{\lambda_{i} \lambda_{j}} y_{j}$ for $1 \leq i<j \leq m, \mathrm{H}_{i i i}=12 \lambda_{i} y_{i}-8 \lambda_{i}^{\frac{3}{2}} y_{i}^{3}$. To summarize, 
Lemma 4 Convergence of the renomalization sequence.

(i)As \& goes to 0, $w_{1, \varepsilon}$ tends to $w_{1}$, solution of the equation:

$$
\Delta_{E} w_{1}(y)+\left[\sum_{k=1}^{m} \lambda_{k}(P) y_{k}^{2}-\Lambda(P)\right] w_{1}(y)+\sum_{1 \leq i \leq j \leq k \leq m} c_{i j k}(P) y_{i} y_{j} y_{k} w_{P}(y)=0
$$

(ii) Up to a multiple of $w_{P}$

$$
\begin{aligned}
w_{1}= & -\sum_{1 \leq i \leq j \leq k \leq m} \frac{c_{i j k}}{16 \sqrt[4]{\lambda_{i}(P) \lambda_{j}(P) \lambda_{k}(P)}} \frac{H_{e_{i}+e_{j}+e_{k}}(y)}{<e_{i}+e_{j}+e_{k}, \sqrt{\lambda(P)}>} \\
& \sum_{1 \leq i<j \leq m}\left[\frac{c_{i i j}}{8 \sqrt[4]{\lambda_{i}^{2}(P) \lambda_{j}^{3}(P)}} H_{e_{j}}(y)+\frac{c_{i j j}}{8 \sqrt[4]{\lambda_{i}^{3}(P) \lambda_{j}^{2}(P)}} H_{e_{i}}(y)\right]
\end{aligned}
$$

\subsubsection{Expansion of the eigenvalue}

It is well known that the eigenvalue $\lambda_{\epsilon}$ has an asymptotic expansion of the type $\Lambda \sqrt{\varepsilon}+$ $\theta \varepsilon+\ldots$ (see [30, 31]). We have already seen that:

$$
\Lambda=\left\{\sum_{n=1}^{m} \sqrt{\lambda_{n}(P)} \mid P \in \operatorname{Crit}(c)\right\}
$$

In this section we want to compute $\theta$ using minimax procedures. Recall that:

$$
\lambda_{\epsilon}=\inf _{u \in H^{1}(V)-\{0\}} \frac{\int_{V}\left[\epsilon\|\nabla u\|_{g}^{2}+c u^{2}\right] d v o l_{g}}{\int_{V} u^{2} d v o l_{g}}
$$

Let us set:

$$
\theta_{\epsilon}=\frac{\lambda_{\epsilon}-\min _{V} c-\sqrt{\epsilon} \Lambda}{\epsilon}
$$

Then:

$$
\theta_{\epsilon}=\inf _{u \in H^{1}(V)-\{0\}} \frac{\int_{V}\left[\|\nabla u\|_{g}^{2}+\frac{c-m i n c-\sqrt{\epsilon} \Lambda}{\epsilon} u^{2}\right] d v o l_{g}}{\int_{V} u^{2} d v o l_{g}}
$$

We shall prove that as $\varepsilon$ goes to $0, \theta_{\epsilon}$ converges and compute its limit $\theta$. It will follow that the limit measures are supported in a subset of $C_{\min \min }$. We shall estimate the variational quotient

$$
\theta_{\epsilon}=\tilde{Q}\left(u_{\epsilon}\right)=\frac{\int_{V_{m}}\left[\left\|\nabla u_{\epsilon}\right\|_{g}^{2}+\frac{c-m i n c-\sqrt{\epsilon} \Lambda}{\epsilon} u_{\epsilon}^{2}\right] d v o l_{g}}{\int_{V_{m}} u_{\epsilon}^{2} d v o l_{g}} .
$$


using the previous blow up analysis. Consider a converging subsequence $\left\{\mathrm{u}_{\varepsilon_{n}} \mid n \in \mathbb{N}\right\}, \mathrm{u}_{\varepsilon_{n}}$ normalized $\left(\int_{V_{m}} u_{\epsilon_{n}}^{2} d v o l_{g}=1\right)$, such that

$$
u_{\epsilon_{n}}^{2} d v o l_{g} \rightarrow \sum_{P \in C_{\min }} \gamma_{P} \delta_{P}
$$

where $\gamma_{P_{1}}=1$ for at least one $\mathrm{P}_{1}$. To simplifie the notations we shall drop the index $\mathrm{n}$ for $\varepsilon_{n}$ from now on.

$$
\begin{aligned}
\tilde{Q}\left(u_{\epsilon}\right) & =\sum_{P \in C_{\min }} \int_{B_{P}(\delta)}\left[\left\|\nabla u_{\epsilon}\right\|_{g}^{2}+\frac{c-\operatorname{minc}-\sqrt{\epsilon} \Lambda}{\epsilon} u_{\epsilon}^{2}\right] d v o l_{g} \\
& +\int_{V-\cup_{P \in C_{\min }} B_{P}(\delta)}\left[\left\|\nabla u_{\epsilon}\right\|_{g}^{2}+\frac{c-\operatorname{minc}-\sqrt{\epsilon} \Lambda}{\epsilon} u_{\epsilon}^{2}\right] d v o l_{g}
\end{aligned}
$$

Let $\phi$ be a $\mathrm{C}^{\infty}$ function: $\mathrm{V} \longrightarrow[0,1], 1$ on $\mathrm{V}-\cup_{P \in C_{\min }} \mathrm{B}_{P}(\delta)$ such that support $\phi \cap C_{\min }=\varnothing$. For $\varepsilon$ sufficiently small

$$
\int_{V-\cup_{P \in C_{\min }} B_{P}(\delta)}\left[\left\|\nabla u_{\epsilon}\right\|_{g}^{2}+\frac{c-\operatorname{minc}-\sqrt{\epsilon} \Lambda}{\epsilon} u_{\epsilon}^{2}\right] d v o l_{g} \leq \int_{V}\left[\left\|\nabla u_{\epsilon}\right\|_{g}^{2}+\frac{c-\operatorname{minc}-\sqrt{\epsilon} \Lambda}{\epsilon} u_{\epsilon}^{2}\right] \phi d v o l_{g}
$$

Using the relation 9,

$$
\int_{V}\left[\left\|\nabla u_{\epsilon}\right\|_{g}^{2}+\frac{c-\operatorname{minc}-\sqrt{\epsilon} \Lambda}{\epsilon} u_{\epsilon}^{2}\right] \phi d v o l_{g}=\int_{V}\left[\frac{\lambda-\operatorname{minc}-\sqrt{\epsilon} \Lambda}{\epsilon} \phi-\Delta \phi\right] u_{\epsilon}^{2} d v o l_{g}
$$

Appendix 2 shows that with an appropriate constant $C$

$$
\int_{V}\left[\frac{\lambda-\operatorname{minc}-\sqrt{\epsilon} \Lambda}{\epsilon} \phi-\Delta \phi\right] u_{\epsilon}^{2} d v o l_{g}=o(1) \epsilon^{m / 4} \bar{u}_{\epsilon}^{2}
$$

Hence:

$$
\begin{gathered}
\tilde{Q}\left(u_{\epsilon}\right)=\sum_{P \in C_{\min }} \int_{B_{P}(\delta)}\left[\left\|\nabla u_{\epsilon}\right\|_{g}^{2}+\frac{c-m i n c-\sqrt{\epsilon} \Lambda}{\epsilon} u_{\epsilon}^{2}\right] d v o l_{g}+o(1) \epsilon^{m / 4} \bar{u}_{\epsilon}^{2} \\
\tilde{Q}\left(u_{\epsilon}\right)=\epsilon^{m / 4} \bar{u}_{\epsilon}^{2} \sum_{P \in C_{\min }} \int_{B_{P}(\delta / \sqrt[4]{\varepsilon})}\left[\frac{1}{\sqrt{\epsilon}}\left\|\nabla w_{P, \epsilon}(y)\right\|_{g}^{2}+\frac{(c(y \sqrt[4]{\varepsilon})-m i n c-\sqrt{\epsilon} \Lambda) w_{P, \epsilon}^{2}(y)}{\epsilon}\right] \sqrt{\operatorname{det} g_{\varepsilon}(y)} d y \\
+o(1) \epsilon^{m / 4} \bar{u}_{\epsilon}^{2}
\end{gathered}
$$

The normalization condition implies that

$$
1=\sum_{P \in C_{\min }} \int_{B_{P}(\delta)} u_{\epsilon}^{2} d v o l_{g}+\int_{V-\cup_{P \in C_{\min }} B_{P}(\delta)} u_{\epsilon}^{2} d v o l_{g}
$$




$$
\begin{gathered}
\int_{V-\cup_{P \in C_{\min }} B_{P}(\delta)} u_{\epsilon}^{2} d v o l_{g}=o(1) \epsilon^{m / 4} \bar{u}_{\epsilon}^{2} \\
\int_{B_{P}(\delta)} u_{\epsilon}^{2} d v o l_{g}=\epsilon^{m / 4} \bar{u}_{\epsilon}^{2} \int_{\mathcal{B}_{P}(\sqrt{\rho} / \sqrt[4]{\varepsilon})} w_{P, \epsilon}^{2}(y) \sqrt{\operatorname{det} g(y \sqrt[4]{\varepsilon})} d y
\end{gathered}
$$

Now $\sup |\operatorname{det} g(y \sqrt[4]{\varepsilon})|<+\infty$ and on each compact subset of $\mathbb{R}^{m}$, $\operatorname{det} g(y \sqrt[4]{\varepsilon})$ con$\mathcal{B}_{P}(\sqrt{\rho} / \sqrt[4]{\varepsilon})$

verges uniformly to 1 when $\varepsilon$ goes to0. Hence $\int_{\mathcal{B}_{P}(\sqrt{\rho} / \sqrt[4]{\varepsilon})} w_{P, \epsilon}^{2}(y) \sqrt{\operatorname{det} g(y \sqrt[4]{\varepsilon})} d y$ tends to $\int_{\mathbb{R}^{m}} w_{P}(y)^{2} d y$ as $\varepsilon$ goes to 0 . By Theorem 2

$$
\int_{\mathbb{R}^{m}} w_{P}(y)^{2} d y=(2 \pi)^{\frac{m}{2}} f_{P}^{2} \prod_{k=1}^{m} \lambda_{k}^{-1 / 4}(P) .
$$

Then relations 41,42 imply:

$$
\lim _{\varepsilon \rightarrow 0} \epsilon^{m / 4} \bar{u}_{\epsilon}^{2}=K=1 / \sum_{P \in C_{\min }}(2 \pi)^{\frac{m}{2}} f_{P}^{2} \prod_{k=1}^{m} \lambda_{k}^{-1 / 4}(P)
$$

Now we shall compute the numerator of $\tilde{Q}\left(u_{\epsilon}\right)$.

$$
\begin{array}{r}
\lim _{\varepsilon \longrightarrow 0} \tilde{Q}\left(u_{\epsilon}\right)=K \sum_{P \in C_{\min }} \lim _{\varepsilon \longrightarrow 0} \int_{B_{P}(\delta / \sqrt[4]{\varepsilon})}\left[\frac{1}{\sqrt{\epsilon}}\left\|\nabla w_{P, \epsilon}(y)\right\|_{g}^{2}+\frac{(c(y \sqrt[4]{\varepsilon})-m i n c-\sqrt{\epsilon} \Lambda) w_{P, \epsilon}^{2}(y)}{\epsilon}\right] \\
\times \sqrt{\operatorname{det} g(y \sqrt[4]{\varepsilon})} d y .(44)
\end{array}
$$

To simplify the notations below set

$$
\begin{gathered}
q(y)=\sum_{k=1}^{m} \lambda_{k}(P) y_{k}^{2}-\Lambda \\
\int_{B_{P}(\delta / \sqrt[4]{\varepsilon})}\left[\frac{1}{\sqrt{\epsilon}}\left\|\nabla w_{P, \epsilon}(y)\right\|_{g}^{2}+\frac{(c(y \sqrt[4]{\varepsilon})-m i n c-\sqrt{\epsilon} \Lambda) w_{P, \epsilon}^{2}(y)}{\epsilon}\right] \sqrt{\operatorname{det} g(y \sqrt[4]{\varepsilon})} d y=\mathrm{I}+\mathrm{II}+\mathrm{III}+\mathrm{IV} \\
+\mathrm{V}+\mathrm{VI}+\mathrm{VII}
\end{gathered}
$$

where, denoting by $\nabla_{E} f$ the euclidean gradient $\left(\frac{\partial f}{\partial y_{1}}, \ldots, \frac{\partial f}{\partial y_{m}}\right)$

$$
\begin{gathered}
\mathrm{I}=\frac{1}{\sqrt{\epsilon}} \int_{B_{P}(\delta / \sqrt[4]{\varepsilon})}\left[\left\|\nabla_{E} w_{P}(y)\right\|_{\mathbb{R}^{m}}^{2}+q w_{P}(y)^{2}\right] \sqrt{\operatorname{det} g(y \sqrt[4]{\varepsilon})} d y, \\
\mathrm{II}=\frac{2}{\sqrt[4]{\epsilon}} \int_{B_{P}(\delta / \sqrt[4]{\varepsilon})}\left[<\nabla_{E} w_{P}, \nabla_{E} w_{1, \varepsilon}>+q w_{P} w_{1, \varepsilon}\right] \sqrt{\operatorname{det} g(y \sqrt[4]{\varepsilon})} d y,
\end{gathered}
$$




$$
\begin{gathered}
\mathrm{III}=\frac{1}{\sqrt[4]{\epsilon}} \int_{B_{P}(\delta / \sqrt[4]{\varepsilon})} \sum_{1 \leq i \leq j \leq k \leq m} c_{i j k} y_{i} y_{j} y_{k} w_{P}^{2} \sqrt{\operatorname{det} g(y \sqrt[4]{\varepsilon})} d y, \\
\mathrm{IV}=\int_{B_{P}(\delta / \sqrt[4]{\varepsilon})}\left[\left\|\nabla_{E} w_{1, \varepsilon}(y)\right\|_{\mathbb{R}^{m}}^{2}+q w_{1, \varepsilon}(y)^{2}+2 \sum_{1 \leq i \leq j \leq k \leq m} c_{i j k} y_{i} y_{j} y_{k} w_{P} w_{1, \varepsilon}\right] \sqrt{\operatorname{det} g(y \sqrt[4]{\varepsilon})} d y, \\
\mathrm{~V}=\sqrt[4]{\epsilon} \int_{B_{P}(\delta / \sqrt[4]{\varepsilon})} \sum_{1 \leq i \leq j \leq k \leq m} c_{i j k} y_{i} y_{j} y_{k} w_{1, \varepsilon}^{2} \sqrt{\operatorname{det} g(y \sqrt[4]{\varepsilon})} d y \\
\mathrm{VI}=\int_{B_{P}(\delta / \sqrt[4]{\varepsilon})} \sum_{1 \leq i \leq j \leq k \leq l \leq m}^{m} c_{i j k l}(y \sqrt[4]{\varepsilon}) y_{i} y_{j} y_{k} y_{l} w_{P, \varepsilon}^{2} \sqrt{\operatorname{det} g(y \sqrt[4]{\varepsilon})} d y \\
\mathrm{VII}=\int_{B_{P}(\delta / \sqrt[4]{\varepsilon})} \sum_{i, j, k, l=1}^{m} g^{i j k l}(y \sqrt[4]{\varepsilon}) y_{k} y_{l} \frac{\partial w_{P, \varepsilon}}{\partial y_{i}} \frac{\partial w_{P, \varepsilon}}{\partial y_{j}} \sqrt{\operatorname{det} g(y \sqrt[4]{\varepsilon})} d y,
\end{gathered}
$$

where $g^{i j}(x)=\delta^{i j}+\sum_{k, l=1}^{m} g^{i j k l}(x) x_{k} x_{l}$.In particular $g^{i j k l}(0)=\frac{1}{6}\left(R_{i k l j}(P)+R_{j k l i}(P)\right)$. Note that

$$
\sqrt{\operatorname{det} g_{\varepsilon}(y)}=1-\frac{\sqrt{\varepsilon}}{6} R_{i j}(y \sqrt[4]{\varepsilon}) y_{i} y_{j}
$$

where the $R_{i j}$ are $C^{\infty}$ functions on $U_{P}$ and $R_{i j}(0)=\operatorname{Ricc}_{i j}(P)$. It is easy to see that

$$
\begin{aligned}
& \lim _{\varepsilon \longrightarrow 0} \mathrm{VII}=\int_{\mathbb{R}^{m}} \sum_{i, j, k, l=1}^{m} \frac{1}{6}\left(R_{i k l j}(P)+R_{j k l i}(P)\right) y_{k} y_{l} \frac{\partial w_{P}}{\partial y_{i}} \frac{\partial w_{P}}{\partial y_{j}} d y \\
& \lim _{\varepsilon \longrightarrow 0} \mathrm{VI}=\int_{\mathbb{R}^{m}} \sum_{1 \leq i \leq j \leq k \leq l \leq m}^{m} c_{i j k l}(0) y_{i} y_{j} y_{k} y_{l} w_{P}^{2} d y \\
& \lim _{\varepsilon \longrightarrow 0} \mathrm{~V}=0 \\
& \lim _{\varepsilon \longrightarrow 0} \operatorname{IV}=\int_{\mathbb{R}^{m}}\left[\left\|\nabla_{E} w_{1}(y)\right\|_{\mathbb{R}^{m}}^{2}+q w_{1}(y)^{2}+2 \sum_{1 \leq i \leq j \leq k \leq m} c_{i j k} y_{i} y_{j} y_{k} w_{P} w_{1}\right] d y
\end{aligned}
$$

Using equation 39 satisfied by $w_{1}$,

$$
\begin{gathered}
\lim _{\varepsilon \longrightarrow 0} \mathrm{IV}=\int_{\mathbb{R}^{m}} \sum_{1 \leq i \leq j \leq k \leq m} c_{i j k} y_{i} y_{j} y_{k} w_{P} w_{1} d y \\
\mathrm{III}=\frac{1}{\sqrt[4]{\epsilon}} \int_{B_{P}(\delta / \sqrt[4]{\varepsilon})} \sum_{\substack{1 \leq i \leq j \leq k \leq m \\
c_{i j k}}} c_{i} y_{j} y_{k} w_{P}^{2} d y-\frac{\sqrt[4]{\epsilon}}{6} \int_{B_{P}(\delta / \sqrt[4]{\varepsilon})} \sum_{\substack{1 \leq i \leq j \leq k \leq m \\
1 \leq l, n \leq m}} c_{i j k} R_{\ln }(y \sqrt[4]{\varepsilon}) y_{i} y_{j} y_{k} y_{l} y_{n} w_{P}^{2} d y .
\end{gathered}
$$

For reason of symmetry the first integral is 0 and the second tends to 0 as $\varepsilon \longrightarrow 0$.

$$
\lim _{\varepsilon \longrightarrow 0} \text { III }=0
$$




$$
\mathrm{I}=\frac{1}{\sqrt{\epsilon}} \int_{B_{P}(\delta / \sqrt[4]{\varepsilon})}\left[\left\|\nabla_{E} w_{P}(y)\right\|_{\mathbb{R}^{m}}^{2}+q w_{P}(y)^{2}\right]\left(1-\frac{\sqrt{\varepsilon}}{6} \sum_{i j=1}^{m} R_{i j}(y \sqrt[4]{\varepsilon}) y_{i} y_{j}\right) d y
$$

but

$$
\frac{1}{\sqrt{\epsilon}} \int_{B_{P}(\delta / \sqrt[4]{\varepsilon})}\left[\left\|\nabla_{E} w_{P}(y)\right\|_{\mathbb{R}^{m}}^{2}+q w_{P}(y)^{2}\right] d y=\frac{1}{\sqrt{\epsilon}} \int_{S_{P}(\delta / \sqrt[4]{\varepsilon})} w_{P}<\nabla_{E} w_{P}, \vec{n}>d A
$$

converges to zero, due the exponential decay. Here $S_{P}(\delta / \sqrt[4]{\varepsilon})$ is the sphere boundary of $B_{P}(\delta / \sqrt[4]{\varepsilon}), \vec{n}$ is the exterior euclidean unit normal to $S_{P}(\delta / \sqrt[4]{\varepsilon})$ and $d A$ the area measure. It is then clear that

$$
\begin{aligned}
\lim _{\varepsilon \rightarrow 0} \mathrm{I}=-\int_{\mathbb{R}^{m}}\left[\left\|\nabla_{E} w_{P}(y)\right\|_{\mathbb{R}^{m}}^{2}+q w_{P}(y)^{2}\right] \frac{1}{6} \sum_{i j=1}^{m} R i c_{i j}(P) y_{i} y_{j} d y \\
\mathrm{II}=\mathrm{II}_{1}+\mathrm{II}_{2} \\
\mathrm{II}_{1}=\frac{2}{\sqrt[4]{\epsilon}} \int_{B_{P}(\delta / \sqrt[4]{\varepsilon})}\left[<\nabla_{E} w_{P}, \nabla_{E} w_{1, \varepsilon}>+q w_{P} w_{1, \varepsilon}\right] d y \\
\mathrm{II}_{1}=\frac{2}{\sqrt[4]{\epsilon}} \int_{S_{P}(\delta / \sqrt[4]{\varepsilon})} w_{1, \varepsilon}<\nabla_{E} w_{P}, \vec{n}>d A \\
\lim _{\varepsilon \longrightarrow 0} \mathrm{II}_{1}=0
\end{aligned}
$$

where an intergration by part leads to a boundary term, which converges to zero.

$$
\mathrm{II}_{2}=-\frac{\sqrt[4]{\varepsilon}}{6} \int_{B_{P}(\delta / \sqrt[4]{\varepsilon})}\left[<\nabla_{E} w_{P}, \nabla_{E} w_{1, \varepsilon}>+q w_{P} w_{1, \varepsilon}\right] \sum_{i j=1}^{m} R_{i j}(y \sqrt[4]{\varepsilon}) y_{i} y_{j} d y
$$

$$
\lim _{\varepsilon \longrightarrow 0} \mathrm{II}_{2}=0 \text {. }
$$

Finally $\lim _{\varepsilon \longrightarrow 0} \int_{B_{P}(\delta / \sqrt[4]{\varepsilon})}\left[\frac{1}{\sqrt{\epsilon}}\left\|\nabla w_{P, \epsilon}(y)\right\|_{g}^{2}+\frac{(c(y \sqrt[4]{\varepsilon})-m i n c-\sqrt{\epsilon} \Lambda) w_{P, \epsilon}^{2}(y)}{\epsilon}\right] \sqrt{\operatorname{det} g(y \sqrt[4]{\varepsilon})} d y$ is equal to $\lim _{\varepsilon \longrightarrow 0} \mathrm{I}+\lim _{\varepsilon \longrightarrow 0} \mathrm{IV}+\lim _{\varepsilon \longrightarrow 0} \mathrm{~V}+\lim _{\varepsilon \longrightarrow 0} \mathrm{VI}$. Lengthy but straightforward computations show that with $\sqrt[4]{\lambda(P)}=\prod_{n=1}^{m} \sqrt[4]{\lambda_{n}(P)}$ :

$$
\begin{gathered}
\lim _{\varepsilon \longrightarrow 0} \mathrm{I}+\lim _{\varepsilon \longrightarrow 0} \mathrm{~V}=-f_{P}^{2} \frac{\pi^{\frac{m}{2}}}{\sqrt[4]{\lambda(P)}}\left[\frac{R(P)}{4}+\frac{1}{12} \sum_{1 \leq i, j \leq m} R_{i j i j}(P) \sqrt{\frac{\lambda_{i}(P)}{\lambda_{j}(P)}}\right] \\
\lim _{\varepsilon \longrightarrow 0} \mathrm{VI}=\frac{1}{4} f_{P}^{2} \frac{\pi^{\frac{m}{2}}}{\sqrt[4]{\lambda(P)}} \sum_{1 \leq i \leq j \leq m} \frac{c_{i i j j}(P)}{\sqrt{\lambda_{i}(P) \lambda_{j}(P)}}+\frac{1}{2} \sum_{i=1}^{m} \frac{c_{i i i i}(P)}{\lambda_{i}(P)} .
\end{gathered}
$$


The computation of $\quad \lim _{\varepsilon \longrightarrow 0}$ IV is more involved, where

$$
L_{P}=\Delta_{E}+\sum_{n=1}^{m} x_{n}^{2} \lambda_{n}(P)-\Lambda(P) .
$$

Then

$$
\begin{aligned}
\sum_{1 \leq i \leq j \leq k \leq m} c_{i j k} y_{i} y_{j} y_{k} w_{P} & =f_{P} \sum_{1 \leq i \leq j \leq k \leq m} \frac{c_{i j k}}{8 \sqrt[4]{\lambda_{i}(P) \lambda_{j}(P) \lambda_{k}(P)}} H_{e_{i}+e_{j}+e_{k}}(y)+ \\
f_{P} \sum_{1 \leq i<j \leq m} & {\left[\frac{c_{i i j}}{4 \sqrt[4]{\lambda_{i}^{2}(P) \lambda_{j}(P)}} H_{e_{j}}(y)+\frac{c_{i j j}}{4 \sqrt[4]{\lambda_{i}(P) \lambda_{j}^{2}(P)}} H_{e_{i}}(y)\right] . }
\end{aligned}
$$

After lengthy computations we get

$$
\begin{gathered}
\int_{\mathbb{R}^{m}} \sum_{1 \leq i \leq j \leq k \leq m} c_{i j k} y_{i} y_{j} y_{k} w_{P} w_{1} d y=-\frac{\pi^{\frac{m}{2}} f_{P}^{2}}{\sqrt[4]{\lambda(P)}}(A(P)+B(P)+C(P)) . \\
A(P)=\sum_{1 \leq i<j<k \leq m} \frac{c_{i j k}^{2}}{16 \sqrt{\lambda_{i}(P) \lambda_{j}(P) \lambda_{k}(P)}} \frac{1}{<e_{i}+e_{j}+e_{k}, \sqrt{\lambda(P)}>} \\
B(P)= \\
\sum_{0 \leq i<j \leq m}\left[\frac{c_{i i j}^{2}}{8 \lambda_{i}(P) \sqrt{\lambda_{j}(P)}}+\frac{c_{i j j}^{2}}{8 \lambda_{j}(P) \sqrt{\lambda_{i}(P)}}\right] \\
\sum_{0 \leq i<j \leq m} \frac{c_{i i j}^{2}+c_{i j j}^{2}}{16 \lambda_{i}(P) \lambda_{j}(P)}+\sum_{i=1}^{m} \frac{c_{i i i}^{2}}{8 \lambda_{i}^{2}(P)}
\end{gathered}
$$

Theorem 4 The expansion of the eigenvalue in power of $\sqrt{\varepsilon}$ is up to order three

$$
\lambda_{\epsilon}=\min _{V} c+\Lambda \sqrt{\varepsilon}+\theta \varepsilon+o(\varepsilon)
$$

where

$$
\begin{gathered}
C_{\min }=\{P \text { such that } c(P)=\min c\} \\
\Lambda=\inf \left[\sum_{n=1}^{m} \sqrt{\lambda_{n}(R)} \mid R \in C_{\min }\right], \\
C_{\min \min }=\left\{P \text { such that } \Lambda(P)=\sum_{n=1}^{m} \sqrt{\lambda_{n}(P)} \mid P \in C_{\min }\right\}
\end{gathered}
$$

and

$$
\begin{aligned}
& \theta=\min _{P \in C \min \min } \frac{\pi^{\frac{m}{2}} K f_{P}^{2}}{\sqrt[4]{\lambda(P)}}\left[-\frac{R(P)}{4}-\frac{1}{12} \sum_{i, j} R_{i j i j}(P) \sqrt{\frac{\lambda_{i}(P)}{\lambda_{j}(P)}+}\right. \\
& \left.\frac{1}{4} \sum_{i \leq j} \frac{c_{i i j j}(P)}{\sqrt{\lambda_{i}(P) \lambda_{j}(P)}}+\frac{1}{2} \sum_{i} \frac{c_{i i i i}(P)}{\lambda_{i}(P)}+A(P)+B(P)+C(P)\right]
\end{aligned}
$$




\section{Remarks.}

1. The sequence $u_{\epsilon}$ is in $H^{m}$ but not in $H^{s}$, for $s>m=\operatorname{dim} V$. This is exactly the critical case in the Sobolev embedding theorem. That why the limits of $u_{\epsilon}$ as $\epsilon$ goes to zero are measures but not functions.

2. The coefficients of the limit measure depends on two factors: one is the second derivative of the potential at some points of $C_{\min \min }$ and second on the coefficients $\alpha$, that measure the ratio of the local maximum versus the global maximum.

3. Actually, we conjecture that if two points $P_{1}$ and $P_{2}$ are always charged and under some conditions of analycity on the metric, any small neighborhood $V_{1}$ of $P_{1}$ is conjugated to a neighborhood $V_{2}$ of $P_{2}$ by an isometry. Such an isometry should leave invariant the blow-up equation defined on the manifold and satisfies by $w_{\epsilon}$. If in $V_{1}$, there exists a subsequence of local maximum, then such subsequence is also present in $V_{2}$, which garantee the concentration in both places. In some sense, when the concentration occurs at those points for any subsequences, it implies the existence of hidden symmetries.

4. Suppose that the potential has only two wells centered at $P_{1}$ and $P_{2}$ that are charged. Obviously when the coefficients of concentration are not unique, the set of possible limit coefficients $\alpha_{1}, \alpha_{2}$, where $\mu=\alpha_{1} \delta_{P_{1}}+\alpha_{2} \delta_{P_{2}}$, are restricted to the intervals:

$$
\alpha_{1} \in\left[c_{1} / c_{1}+c 2,1\right] \text { and } \alpha_{2} \in\left[0, c_{2} /(c 1+c 2)\right] \text {. }
$$

Indeed one of the coefficient, say $\alpha_{1}$ characterize the convergence of the absolute maximum.

\subsection{Removing the degeneracy}

In the previous analysis we have defined two local characteristics (quantities that are functions of $g$ and $c), \sum_{n=1}^{m} \lambda_{n}(P)$ and $-\mathcal{D}(P)+\mathcal{C}(P)$ which have properties that the minimum of the first one on $C_{\text {min }}$ gives the first coefficient of the developpment of $\lambda_{\epsilon}$ in the power of $\sqrt{\epsilon}$,

$$
\lambda_{\epsilon}=a_{0}+a_{1} \sqrt{\epsilon}+. .+a_{n} \sqrt{\epsilon^{n}}+\ldots
$$

$C_{\text {minmin }}$ is the subset of $C_{\min }$ where the characteristic achieves its minimum. The second coefficient of the developpment of $\lambda_{\epsilon}$ is equal to the minimum of the second characteristic on the set $C_{\text {minmin }}$. We could continue this process and define a sequence of characteristics $\chi_{2}, \chi_{3}, \chi_{4}, \ldots\left(\chi_{0}=\min _{V_{m}} c, \chi_{1}=\Lambda\right.$ and $\left.\chi_{2}=\theta\right)$ and subsets of $C_{m i n}, C_{3}, C_{4}, C_{5} \ldots$ having the following property, for any integer $n$ :

- $\chi_{n}$ is a function defined on $C_{n}$ and depends only on the covariant derivatives of $c$ and $g$

- the $\mathrm{n}^{\text {th }}$ coefficient $c_{n}$ is equal $\min _{C_{n}} \chi_{n}$. 
- $C_{n+1}=\left\{P \in C_{n} \mid \chi_{n}(P)=a_{n}\right\}$.

Since the set $C_{\min }$ is finite, the process becomes stationnary after a finite number of steps i.e. $C_{n}=C_{n+1}$ and $\chi_{n}$ is constant on $C_{n}$, for n larger than $\bar{n}$, some integer. Now there are two possibilities. Either $C_{\bar{n}}$ is reduced to a single point, in which case, this is the only point that is charged or the so-called degenerate case, see (31] page 304) where $C_{\bar{n}}$ has more than one point.

The knowledge of the expansion of $\lambda_{\epsilon}$ is not enough to compute the value of the concentration coefficient. It is an open problem to compute precisely this value in terms of the geometry and the extracted subsequence.

Finally we conjecture that for any points $P_{1}, P_{2}$ in $C_{\bar{n}}$, there exists a isometry $\phi: \Omega_{1} \rightarrow$ $\Omega_{2}$, where $\Omega_{i}$ is an open neighborhood of $P_{i}$ such that denoting by $c_{1}, c_{2}$ the restrictions of $c$ to $\Omega_{1}, \Omega_{2}$ respectively, $c_{2} \circ \phi=c_{1}$ and $\left.w_{\epsilon}\right|_{\Omega_{2}}=\left.w_{\epsilon}\right|_{\Omega_{1}} \circ \phi$.

\section{Blow up analysis with a gradient vector field}

In this section, we study the behavior of the sequence of first eigenfunctions, when the vector field $b$ is the gradient of a Morse function, $b=\nabla \phi$, with respect to the metric g. It is possible to describe explicitly the set of limit measures and we will see how the supports of these limit measures coincide with those suggested by the analysis of the Topological Pressure ([22]). More precisely, the potential $c$ and the field $b=\nabla \phi$ interact to restrict the limits of the eigenfunctions, to certain subsets of the critical set of $b$. The limits depend on the couple of functions $(c, \phi)$.

Somewhat surprisingly not all the attractors points are charged. It depends on the topological pressure (see [22]) and can be understood as follows: $c$ acts as a killing term (see [29, 15]) and can destroy all the particles near an attractor;

We begin analyzing the problem. The eigenfunction satisfies the partial differential equation and normalisation condition

$$
\begin{aligned}
\epsilon \Delta_{g} u_{\epsilon}+\left(\nabla \phi, \nabla u_{\epsilon}\right)_{g}+c u_{\epsilon} & =\lambda_{\epsilon} u_{\epsilon} \\
\int_{V_{n}} u_{\epsilon}^{2} d v o l_{g} & =1 .
\end{aligned}
$$

We denote by $\operatorname{Sing}(\mathrm{b})$ the set of singular points of the field $b$. When $\mathrm{b}$ is the gradient of a function $\phi$, these points are just the critical points of $\phi$.

\subsection{What do we learn from the Topological Pressure}

The topological pressure can be defined as follows:

$$
\operatorname{Pr}=\inf _{P \in \operatorname{Si} n g(b)}\left\{c(P)-\sum_{i=1}^{m} \min \left(0, \operatorname{Re} \lambda_{i}(P)\right\}\right.
$$


where $\lambda_{i}(P), 1 \leq i \leq m$, are the eigenvalues of the linear part of the field $\operatorname{grad} \phi$ at the point $P$. The definition for Morse-Smale fields can be found in ([20]), for more general vector fields in (22]). Because the infimum in (48) is taken over a finite number of points, it is attained somewhere but not necessarily at an attractor (that is clear from the formula).

It will be useful to perform a gauge transformation:

$$
\begin{aligned}
v_{\epsilon} & =u_{\epsilon} e^{-\phi / 2 \epsilon} \\
b & =\nabla \phi
\end{aligned}
$$

By this gauge transformation, equation (46) is transformed into

$$
\epsilon \Delta_{g} v_{\epsilon}+\left(c+\frac{\Delta_{g} \phi}{2}+\frac{\|\nabla \phi\|_{g}^{2}}{4 \epsilon}\right) v_{\epsilon}=\lambda_{\epsilon} v_{\epsilon}
$$

with the new condition of normalization $\int_{V} v_{\epsilon}^{2} d v o l_{g}=1$. We will analyze the set of limits of the measures $v_{\epsilon}^{2} d v o l_{g}$ as $\varepsilon$ tends to 0 .

\section{2 blow-up Analysis}

It has been proved in the second section that the sequence $\sup _{\epsilon} v_{\epsilon}$ converges to infinity as $\epsilon$ goes to zero and the sequence $v_{\epsilon}$ converges uniformly to zero on every compact set that does not intersect the singular set of the field. As in the field- free case, if $\mathrm{P} \in \operatorname{Sing}(b)$ we choose a normal coordinate system $\left(\mathrm{x}_{1}, \ldots \mathrm{x}_{m}\right): \mathrm{U} \longrightarrow \mathbb{R}$, centered at $\mathrm{P}$, defined on a domain U such that:

1) $\mathrm{x}_{1} \times \ldots \times \mathrm{x}_{m}(U)$ contains the closed ball $\mathrm{B}_{P}(\delta)$ centered at $\mathrm{P}$ and having radius $\delta>0$.

2)for all $\mathrm{i}, \mathrm{j}, 1 \leq \mathrm{i}, \mathrm{j} \leq \mathrm{m}, \frac{\partial^{2} \phi}{\partial x_{i} \partial x_{j}}(P)=\lambda_{i}(P) \delta_{i j}$.

3) $\mathrm{U} \cap \operatorname{Sing}(\mathrm{b})=\{\mathrm{P}\}$

On the magnified set $\frac{1}{\sqrt{\varepsilon}} \mathrm{x}_{1} \times \ldots \times \mathrm{x}_{m}(U)$, we can define the function $w_{\varepsilon}: w_{\varepsilon}(y)=\frac{v_{\varepsilon}(y \sqrt{\varepsilon})}{\bar{v}_{\varepsilon}}$ where $\bar{v}_{\varepsilon}=\max _{c} v_{\varepsilon}$.

The main theorem of this section is the following:

Theorem 5 - (i)Suppose that the vector field $b$ is gradient-like, $b=\nabla \phi$, where $\phi$ has the expansion near each critical point: $\phi(P)=\phi(P)+\sum_{i=1}^{m} \lambda_{i}(P)\left(x^{i}\right)^{2}+O\left(\|x\|_{g}^{3}\right)$. The set $\mathcal{M}$ of all possible limit measures of the sequence $v_{\epsilon}^{2} d v o l_{g}$, is described by:

$$
\mathcal{P}=\left\{\mu \in M(V) \mid \mu=\sum_{P \in S} c_{P} \delta_{P}, c_{P} \geq 0, \sum_{P \in S} c_{P}=1\right\}
$$

$S$ is set the critical points of $b$, where the Topological Pressure is achieved.

- (ii) When all the points of $S$ are maximally charged, the coefficients $c_{P}$ can be computed explicitly, $c_{P}=\frac{\prod_{1}^{m}\left|\lambda_{i}(P)\right|^{-1 / 2}}{\sum_{i \in \Lambda} \prod_{1}^{m}\left|\lambda_{i}(P)\right|^{-1 / 2}}$ 
- When a partial set $\widehat{S}$ of $S$ is maximally charged, the formula changes to:

$$
c_{P}=\frac{\prod_{1}^{m}\left|\lambda_{i}(P)\right|^{-1 / 2}}{\sum_{P \in \widehat{S}} \prod_{1}^{m}\left|\lambda_{i}(P)\right|^{-1 / 2}}
$$

Lemma 5 For each $\varepsilon$, let us denote by $\mathcal{M}_{\varepsilon}$, the set of all maximum points of $v_{\varepsilon}$ in the manifold.There exists a constant $A$ depending only on $c$ and $b$, such that:

$$
\sup _{q \in \mathcal{M}_{\varepsilon}} d_{g}^{2}(q, \operatorname{Sing}(b)) \leq A \epsilon
$$

It follows from this that the set of limit points of the set of maximum points of $v_{\varepsilon}$ is contained in $\operatorname{Sing}(b)$.

Proof.

If we denote by $Q_{\epsilon}$ a sequence of maximum points of $v_{\epsilon}$. Using the Maximal Principle, applied to equation (46), it is possible to estimate the velocity at which the sequence of maximal points $Q_{\epsilon}$ converges to a critical point of the field. Indeed, we obtain that

$$
\left(c\left(Q_{\epsilon}\right)+\frac{\Delta \phi\left(Q_{\epsilon}\right)}{2}+\frac{\left\|\nabla \phi\left(Q_{\epsilon}\right)\right\|^{2}}{4 \epsilon} \leq \lambda_{\epsilon} \leq C\right.
$$

for some constant $\mathrm{C}$. This implies that $\left\|\nabla \phi\left(Q_{\epsilon}\right)\right\|^{2} \leq 4 C \epsilon$. Now it is easy to see that there exists a constant $\Gamma$ depending only on $\mathrm{c}$ such that for $\mathrm{P} \in \mathrm{V}, \mathrm{d}_{g}^{2}(\mathrm{P}, \operatorname{Sing}(\mathrm{b})) \leq \Gamma\left(\mathrm{c}(\mathrm{P})-\min _{V}\right.$ c). Take $\mathrm{A}=4 \Gamma \Lambda$. Then $d_{g}^{2}\left(Q_{\epsilon}, \operatorname{Si} n g(b)\right) \leq A \epsilon$. The lemma shows that $Q_{\epsilon}$ tends to $\operatorname{Sing}(b)$ at least as fast as $\sqrt{\epsilon}$. In fact, theorem below shows that it tends faster.

\section{Theorem 6 Concentration with a gradient vector field.}

(i) For any $P \in C_{\min }$, any sequence of $v_{\varepsilon}$ 's, with $\varepsilon$ tending to 0 , contains a subsequence $\left\{v_{\varepsilon_{n}}\right\}$, such that the sequence of blown-up functions $\frac{v_{\varepsilon_{n}}\left(y \sqrt[4]{\varepsilon_{n}}\right)}{\bar{v}_{\varepsilon_{n}}}$ at $P$ converges to a function $w: \mathbb{R}^{m}->\mathbb{R}_{+}$, both in the $L^{2}$ norm and the $C^{\infty}$ topology.

(ii) w satisfies the equation:

$$
\begin{gathered}
\Delta_{E} w+\left(c(P)+\frac{\Delta \phi}{2}(P)+\sum \lambda_{i}(P)\left(x^{i}\right)^{2}\right) w=\lambda w \\
0<w \leq \max _{\mathbb{R}^{m}} w=1 .
\end{gathered}
$$

(iii) When the eigenvalue $\lambda$ is equal to the Topological Pressure at $P$, the solution $w$ is nonzero and given explicitly by:

$$
w(x)=\prod_{i=1}^{m} e^{-\left|\lambda_{i}(P)\right| x_{i}^{2} / 2}
$$

in particular,

$$
\int_{\mathbb{R}^{m}} w^{2}(x)=\frac{\pi^{m / 2}}{\prod_{i=1}^{m}\left|\lambda_{i}\right|^{1 / 2}}
$$


Moreover,

$$
\lim _{\epsilon \rightarrow 0} \epsilon^{m / 2} \sup _{V} v_{\epsilon}^{2}=K(c, \phi)
$$

where $K(c, \phi)$ is a positive constant depending only on the functions $c, \phi$.

(iv)If all the singular points at which the topological pressure is attained (set denoted by "top") are maximally charged,

$$
K(c, \phi)=\frac{\pi^{m / 2}}{\sum\left\{\prod_{i=1}^{m}\left|\lambda_{i}(P)\right|^{-1 / 2} \mid P \in \operatorname{top}\right\}} .
$$

Moreover

$$
\lim _{\epsilon \rightarrow 0} \frac{d_{g}\left(Q_{\epsilon}, P\right)}{\epsilon^{1 / 2}}=0 .
$$

\section{Remark.}

At this stage, let us point out the difference with the pure potential case, studied in the last section. There the scaling factor was $\varepsilon^{1 / 4}$ and the concentration took place on certain minimum points of the potential only. In the present situation, the scaling factor is $\sqrt{\varepsilon}$ and the concentration is determined by the couple field-potential $(\nabla \phi, c)$.

Before proving the theorem, two lemmas are needed. The first one provides estimates of the first eigenvalue $\lambda_{\epsilon}$ and the second, estimates of the decay of renormalized sequence $w_{\epsilon}(x)$.

Lemma 6 The first eigenvalue satisfies the inequality

$$
0<\lambda_{\epsilon} \leq \min \left\{c(P)-\sum_{\left\{i, \lambda_{i}(P)<0\right\}} \lambda_{i}(P) \mid P \in \operatorname{Sin} g(b)\right\}
$$

where Sing (b) is the set of critical points of the field $b=\nabla \phi$ and $\lambda_{i}(P), 1 \leq i \leq m$, are the eigenvalues of the field at the critical point $P$.

\section{Proof.}

This is a consequence of the variational approach. Similarly to the case without field, define the variational quotient

$$
Q_{\epsilon}(v)=\frac{\epsilon \int_{V}\left[\|\nabla v\|_{g}^{2}+c_{\varepsilon} v^{2}\right] d v o l_{g}}{\int_{V} v^{2} d v o l_{g}} .
$$

where $c_{\epsilon}=c+\frac{\Delta_{g} \phi}{2}+\frac{\mid \nabla \phi \|_{g}^{2}}{4 \epsilon}$. Then

$$
\lambda_{\epsilon}=\inf _{v \in H^{1}(V)-\{0\}} Q_{\epsilon}(v)>0
$$


In the neighborhood of a singular point $P$, we have an expansion,

$$
c_{\epsilon}(x)=c(P)+\frac{\Delta_{g} \phi(P)}{2}+\sum_{i=1}^{m} a_{i}(P) x^{i}+\sum_{i=1}^{n} \frac{\lambda_{i}^{2}(P) x_{i}^{2}}{\varepsilon}+\sum_{\leq 1 i \leq j \leq k \leq m} c_{i j k}(x) x_{i} x_{j} x_{k}
$$

where the $c_{i j k}$ are $\mathrm{C}^{\infty}$ functions on $\mathrm{U}_{P}$. In order to get an upper bound for the eigenvalue $\lambda_{\epsilon}$, we estimate the quotient $Q_{\epsilon}$, for the following test function with compact support,

$$
\begin{aligned}
\psi_{\epsilon} & =e^{-\sum \mu_{i}\left(x^{i}\right)^{2} / 2}-e^{-\rho /(2 \epsilon)} \text { in } \mathcal{N}_{P}(\rho) \\
& =0, \text { otherwise }
\end{aligned}
$$

where $\mu_{i}=\frac{\left|\lambda_{i}(P)\right|}{\varepsilon}$ and $\mathcal{N}_{P}(\rho)$ is the connected component of the set $\left\{\mathrm{x}\left|\sum_{i=1}^{m}\right| \lambda_{i} \mid x_{i}^{2} \leq \rho\right\}$ containing 0 and $\rho$ is taken so small that $\mathcal{N}_{P}(\rho) \cap\left\{\mathrm{x} \mid \sum_{i=1}^{m} x_{i}^{2}=\delta^{2}\right\}$ is empty. So $\mathcal{N}_{P}(\rho)$ is contained in $\mathrm{B}_{P}(\delta)$. After computations similar to the ones in Lemma 2 of the section 2. we obtain

$$
\begin{aligned}
Q_{\epsilon}\left(\psi_{\varepsilon}\right) & =c(P)+\frac{\Delta_{g} \phi(P)}{2}+\frac{\epsilon}{2} \sum_{i=1}^{n} \mu_{i}+\sum_{i=1}^{n} \frac{\lambda_{i}(P)^{2}}{2 \epsilon \mu_{i}}+o(\epsilon) \\
& =c(P)+\frac{\Delta_{g} \phi(P)}{2}+\sum_{i=1}^{n}\left|\lambda_{i}(P)\right|+o(\epsilon)
\end{aligned}
$$

Using the fact that $\frac{\Delta_{g} \phi(P)}{2}=-\sum_{i=1}^{n} \lambda_{i}(P)$, we obtain that

$$
Q_{\epsilon}\left(\psi_{\varepsilon}\right) \leq c(P)+\sum_{\lambda_{i}(P)<0} \lambda_{i}(P) .
$$

Since a similar test function can be built in the neighborhood of every critical points, we obtain the estimate of the lemma,

$$
\lambda_{\epsilon} \leq \min _{\{P \in \operatorname{Sin} n g(P)\}}\left[c(P)-\sum\left\{\lambda_{i}(P) \mid 1 \leq i \leq m, \lambda_{i}(P)<0\right\}\right] .
$$

Lemma 7 The blown-up sequence $w_{\epsilon}$, defined by

$$
w_{\epsilon}(x)=\frac{v_{\epsilon}(\sqrt{\epsilon} x)}{\sup _{V_{n}} v_{\epsilon}}
$$

satisfies the following properties:

For all $\left.\varepsilon_{0} \in\right] 0,1[$ :

1. $\sup _{\left[0, \varepsilon_{0}\right]} \int_{B_{P}(\delta / \sqrt[4]{\varepsilon})} w_{\varepsilon}(y)^{2} d y<+\infty$. More generally, for any continuous function $f:[0$, $1] \times \mathbb{R}^{m} \longrightarrow \mathbb{R},(\epsilon, y) \longrightarrow f(\epsilon, y)$, having at most polynomial growth at infinity in $y$ uniformly in $\varepsilon, \sup _{\left[0, \varepsilon_{0}\right]} \int_{B_{P}(\delta / \sqrt{\varepsilon})} f(\epsilon, y) w_{\varepsilon}(y)^{2} d y<+\infty$. 
2. the set of restrictions $\left.\left.w_{\varepsilon}^{2} \mid B_{P}(\delta / \sqrt{\varepsilon}), \varepsilon \in\right] 0,1\right]$, of the $w_{\varepsilon}^{2}$ to the balls $B_{P}(\delta / \sqrt[4]{\varepsilon})$ satisfies the following condition: for any $\eta>0$, there exists a compact $K \subset \mathbb{R}^{m}$ and a $\varepsilon(\eta)>0$ such that

$$
\int_{B_{P}(\delta / \sqrt{\varepsilon})-K} w_{\varepsilon}(y)^{2} d y \leq \eta
$$

for all $\varepsilon \in] 0, \varepsilon(\eta)]$.

\section{Proof of Lemmal7}

The proof is similar as the one given to prove the decay estimate in the case of the pure potential case and is based on the Feynman-Kac integral representation of the solution. The Feynman-Kac formula gives that

$e^{-\mu_{\epsilon} t} v_{\epsilon}(x)=e^{-\mu_{\epsilon} t} E_{x}\left(v_{\epsilon}\left(X_{\epsilon}(t)\right) \chi_{\left(t<\tau_{\epsilon}^{x}\right)} e^{-\int_{0}^{t} c_{\epsilon}\left(X_{\epsilon}(s) d s\right)}\right)+E_{x}\left(e^{-\mu_{\epsilon} \tau_{\varepsilon}^{x}} v_{\epsilon}\left(X_{\epsilon}\left(\tau_{\varepsilon}^{x}\right)\right) \chi_{\left(t>\tau_{\epsilon}^{x}\right)} e^{-\int_{0}^{\tau_{\epsilon}^{x}} c_{\epsilon}\left(X_{\epsilon}(t)\right) d s}\right)$

where we recall that $c_{\epsilon}=c+\frac{\Delta_{g} \phi}{2}+\frac{\|\nabla \phi\|_{g}^{2}}{4 \epsilon}$ and $\mathrm{X}_{\varepsilon}$ is defined in relation (23) $) \cdot \tau_{\varepsilon}^{x}$ has the same meaning as in Section (2). Since the function $c+\frac{\Delta_{g} \phi}{2}$ is bounded, it is enough to estimate $E_{x}\left(e^{-\int_{0}^{t} \frac{|\nabla \phi|^{2}}{4 \epsilon}\left(X_{\epsilon}(s)\right) d s}\right)$. Using the scale change $x \rightarrow \epsilon^{1 / 2} x$, this term can be estimated as in Proposition 24 and the results are similar. Indeed, because $\frac{\left\|\nabla \phi\left(\epsilon^{1 / 2} y\right)\right\|^{2}}{4 \epsilon}=\sum_{n=1}^{m} \lambda_{n}(P)^{2} y_{n}^{2}$, the estimate is valid in the neighborhood of any critical point.

Proof of the theorem. Consider the renormalized sequence $w_{\epsilon}(y)=\frac{v_{\epsilon}(y \sqrt{\epsilon})}{\overline{v_{\epsilon}}}, w_{\epsilon}$ satisfies the partial differential equation:

$$
\begin{aligned}
& \Delta_{g_{\epsilon}} w_{\epsilon}(y \sqrt{\epsilon})+\left(c(y \sqrt{\epsilon})+\frac{\Delta_{g} \phi(y \sqrt{\epsilon})}{2}+\frac{\|\nabla \phi\|_{g}^{2}(y \sqrt{\epsilon})}{4 \epsilon}\right) w_{\epsilon}(y \sqrt{\epsilon})=\lambda_{\epsilon} w_{\epsilon}(y \sqrt{\epsilon}) \text { in } \\
& \frac{1}{\sqrt{\varepsilon}} x_{1} \times \ldots \times x_{m}\left(U_{P}\right) \\
& 0<w_{\epsilon} \leq 1
\end{aligned}
$$

To study the equation [50], note that the coefficients of the partial differential equation 50 converges uniformly on every compact set as $\epsilon$ goes to zero: $g_{\epsilon}(x)=g(y \sqrt{\epsilon})$ converges to the Euclidean metric (see the previous section for more details about the type of convergence, usually in $C^{2, \alpha}$ of any compact).

Since the coefficients of the partial differential equation 50 remain bounded as $\epsilon$ goes to zero. Indeed, due the velocity of convergence of the sequence $Q[6]$

$$
\begin{aligned}
\lim _{\epsilon \rightarrow 0}\left(c(y \sqrt{\epsilon})+\frac{\Delta_{g} \phi(y \sqrt{\epsilon})}{2}\right) & =c(P)+\frac{\Delta_{g} \phi(P)}{2} \\
\lim _{\epsilon \rightarrow 0} \frac{\|\nabla \phi(y \sqrt{\epsilon})\|_{g}^{2}}{4 \epsilon} & =\sum_{n=1}^{m} \frac{\lambda_{n}^{2}(P) y_{n}^{2}}{4}
\end{aligned}
$$


Classical elliptic estimates show that $w_{\epsilon}$ converges uniformly on every compact set of $\mathbb{R}^{m}$ to a solution $w$ of

$$
\begin{aligned}
& \Delta_{E} w(y)+\left(c(P)+\frac{\Delta_{g} \phi}{2}(P)+\sum_{n=1}^{m} \lambda_{n}^{2}(P) y_{n}^{2}\right) w(y)=\lambda w(y), \text { on } \mathbb{R}^{m} \\
& 0<w \leq \max _{\mathbb{R}^{m}} w \leq 1 .
\end{aligned}
$$

Let $Q^{*}$ be a limit point of the sequence $\frac{Q_{\epsilon}}{\sqrt{\epsilon}}$, since $w_{\epsilon}$ converges uniformly on every compact to $w$. $w_{\epsilon}\left(\frac{Q_{\epsilon}}{\sqrt{\epsilon}}\right)$ converges to $w\left(Q^{*}\right)$. Hence $w\left(Q^{*}\right)=1$. Because $w$ belongs to $L_{2}\left(\mathbb{R}^{n}\right)$ by Lemma 7 and is not zero, $w$ an eigenfunction of $L_{Q}$ in $L_{2}\left(\mathbb{R}^{n}\right)$ associated to the eigenvalue $\lambda$.

Moreover, using the results of lemma 6.

$$
\lambda-\left(c(P)+\frac{\Delta \phi}{2}(P)\right) \leq \sum_{n=1}^{m}\left|\lambda_{n}(P)\right|
$$

We will now prove, in order for the function $w$ to be nonzero, necessarily $\lambda$ is equal to the Topological Pressure.

In order to prove that $\mu=\lambda-\left(c(P)+\frac{\Delta \phi}{2}(P)\right)=\sum_{n=1}^{m}\left|\lambda_{n}(P)\right|$, one must study the spectrum of the self-adjoint operator defined by $L_{P}=\Delta_{E}+\sum_{n=1}^{m} \frac{\lambda_{n}^{2}(P) x_{n}^{2}}{4}$ in $L_{2}\left(\mathbb{R}^{n}\right)$. This operator is compact and has a discrete spectrum. For all these, see [27]. Using the arguments in the proofs of the main theorem in Section 2.4 we know that the eigenvalues and eigenfunctions of the operator defined by $L_{P}$ in $L_{2}\left(\mathbb{R}^{n}\right)$ are respectively

$$
\sum_{1}^{n}\left(2 \alpha_{i}+1\right) \frac{\left|\lambda_{i}(P)\right|}{2}, \alpha \in \mathbb{Z}_{+}^{n}
$$

and

$$
H_{\alpha}(x)=\prod_{1}^{n} h_{\alpha_{i}}\left(y_{i}\right) e^{-\frac{\lambda_{i}(k)}{2}\left(y_{i}\right)^{2}} \text { where } h_{k}(x)=e^{x^{2} / 4} \frac{d^{k}}{d x^{k}}\left\{e^{-x^{2} / 4}\right\} .
$$

The lowest eigenvalue of $L_{P}$ is

$$
\Lambda_{1}=\sum_{1}^{n}\left|\lambda_{i}(P)\right|
$$

Inequality 53 shows that the eigenvalue $\lambda-\left(c(P)+\frac{\Delta \phi}{2}(P)\right)$ of $L_{P}$ in $L_{2}\left(\mathbb{R}^{n}\right)$ is at most equals to $\lambda_{1}$. Hence it is equal to $\lambda_{1}$ and the corresponding eigenfunction $w$ is a positive multiple of $H_{0}$. Since the only maximum point of $H_{0}$ is $0, Q^{*}=0$ and $w=H_{0}(0)$.

In particular, the previous analysis shows that

$$
\lim _{\epsilon \rightarrow 0} \lambda_{\epsilon}=\left(c(P)+\frac{\Delta \phi}{2}(P)\right)+\Lambda_{1}=\text { Topological Pressure. }
$$

This implies that a point $\mathrm{P}$ is not charged if the Topological Pressure is not attained at P. 
Using the exponential decay of the last lemma, at the point $P$, where the Topological Pressure is attained, we have

$$
\int_{B_{P}(\delta)} v_{\epsilon}^{2} d v o l_{g}=\epsilon^{m / 2} \bar{v}_{\epsilon}^{2} \int_{B_{Q_{k}}(\delta / \epsilon)} w_{\epsilon}^{2} d v o l_{g_{\epsilon}} .
$$

Since the sequence $w_{\epsilon}$ converges in $L_{2}$, this implies that the sequence $\epsilon^{m / 2} \bar{v}_{\epsilon}^{2}$ converges and we get,

$$
\lim _{\epsilon \rightarrow 0} \epsilon^{m / 2} \bar{v}_{\epsilon}^{2}=C(c, \phi) .
$$

The concentration coefficient $c_{k}$ can be computed, if we denote

$$
c_{P}=\lim _{\epsilon \rightarrow 0} \int_{B_{P}(\delta)} v_{\epsilon}^{2} d v o l_{g}=K(c, \phi) \int_{\mathbb{R}^{m}} w_{Q}^{2} .
$$

Using the $L_{2}$ normalization conditions, the Blow up analysis and the fact that the sequence $v_{\epsilon}$ converges to zero on any compact sets that does not intersect the critical points where the topological pressure is attained, we get,

$$
1=K(c, \phi) \sum_{P \in S} \int_{\mathbb{R}^{m}} w_{P}^{2}+o(1)
$$

which proves the last part of the theorem. We shall omit the proof of statement (iv) which is similar to the one given at the end of section2.

\section{Remarks:}

1. Can one find explicitly the asymptotic expansion of $\lambda_{\epsilon}$,

$$
\lambda_{\epsilon}=\sum_{k=0}^{\infty} c_{k} \epsilon^{k / 2}
$$

2. We proved earlier that $c_{0}$ is equal to the Topological Pressure and captures enough information to locate the supports of the possible limit measures. This is surprising because it was not true in the pure potential case.See the section(2.7)

\section{The general case for the first eigenfunction prob- lem}

In this section, we shall consider a special class of vector fields $b$ such that the limits of the first eigenfunctions $u_{\epsilon}$ concentrate on the limit sets of $b$. In particular if the limit sets of the vector field contains limit cycles, we prove that the weak limits of $u_{\epsilon}$ as $\epsilon$ tends to zero, concentrate on these limit cycles. when a

Here we will consider the following class of vector fields : $b=\operatorname{grad}_{g} \mathcal{L}+\Omega$ where $\Omega$ is a Morse-Smale vector field and $\mathcal{L}$ is a special type of Lyapunov function of $\Omega$. We will study the behavior of the first eigenfunction for the operator $L_{\epsilon}$ the drift of which is belongs to this class. We will need the following lemma proved in the appendix. 
Lemma 8 Given a Morse Smale field $\Omega$ there exists $C^{\infty}$ Lyapunov functions for $\Omega$, taking the value zero on all the repulsors of $\Omega$ and such that the function $\Psi(\mathcal{L})=\frac{1}{4}\left(\|\nabla \mathcal{L}\|_{g}^{2}+\frac{1}{2}<\right.$ $\left.\operatorname{grad} \mathcal{L}, \Omega>_{g}\right)$ is positive except on the recurrent set of $\Omega$ where it is zero.

Definition 6 A Lyapunov function with the property stated in lemma 8 will be called a special Lyapunov function.

The main theorem can now be stated:

Theorem 7 On a compact orientable Riemannian manifold $V_{m}$, consider a Morse-Smale vector field $\Omega$ whose recurrent set consists of the stationary points $P_{1}, \ldots, P_{M}$ and of the periodic orbits $\Gamma_{1}, \ldots, \Gamma_{N}$. $\mathcal{L}$ denotes a special Lyapunov function.

For $\epsilon>0$ let $\lambda_{\epsilon}, u_{\epsilon}$ denotes respectively the first eigenvalue and the associated eigenfunction of the operator

$$
L_{\epsilon}=\epsilon \Delta_{g}+b \nabla+c, \text { on } V_{m}
$$

Then the weak limits of the family of probability measures

$$
\frac{e^{-\mathcal{L} / \epsilon} u_{\epsilon}^{2} d V_{g}}{\int_{V} e^{-\mathcal{L} / \epsilon} u_{\epsilon}^{2} d V_{g}}
$$

are of the form

$$
\nu=\sum_{j=1}^{N} \mu_{j}+\sum_{i=1}^{M} \gamma_{P_{i}} \delta_{P_{i}}
$$

where the measures $\mu_{j}$ are supported by the limit cycle $\Gamma_{j}$ and the $P_{i}$ 's $1 \leq i \leq N$ are the critical points of $b$. the $\gamma_{P_{i}}$ are scalars.

$$
1=\sum_{j=1}^{N} \int_{\Gamma_{j}} \mu_{j}+\sum_{i=1}^{M} \gamma_{P_{i}} .
$$

\section{Remark.}

In a future paper [14, we shall prove that for an appropriate choice of Lyapunov function, no concentration can occur on a set of isolated points on the cycle.

point where the topological pressure is found

\subsubsection{Proof of theorem 2}

The proof of theorem 2 will occupy the next two sections. Consider the following transformation $u \longrightarrow v$.

$$
\begin{aligned}
& v=e^{-\frac{\phi}{2 \epsilon}} u \\
& u=e^{\frac{\phi}{2 \epsilon}} v
\end{aligned}
$$


and the second order differential operator $L_{\varepsilon}^{\prime}$ :

$$
L_{\varepsilon}^{\prime} v=\varepsilon e^{-\frac{\phi}{2 \varepsilon}} L_{\varepsilon}\left(e^{\frac{\phi}{2 \epsilon}} v\right)
$$

It is easy to see that:

$$
\Delta_{g} u=e^{\frac{\phi}{2 \epsilon}}\left[\left(\Delta_{g} v-\frac{1}{\epsilon}<\nabla \phi, \nabla v>_{g}+v_{\epsilon}\left(\frac{\Delta_{g} \phi}{2 \epsilon}-\frac{\|\nabla \phi\|_{g}^{2}}{4 \epsilon^{2}}\right)\right]\right.
$$

and

$$
<\nabla u, b>_{g}=e^{\frac{\phi}{2 \epsilon}}\left[\left\langle\nabla v, b>_{g}+v \frac{<\nabla \phi, b>_{g}}{2 \epsilon}\right]\right.
$$

Finally,

$$
L_{\epsilon}^{\prime}(v)=e^{\frac{\phi}{2 \epsilon}}\left[\epsilon \Delta_{g} v+<b-\nabla \phi, \nabla v>_{g}+v\left(c+\frac{<\nabla \phi, b>_{g}}{2 \epsilon}+\frac{\Delta_{g} \phi}{2}-\frac{\|\nabla \phi\|_{g}^{2}}{4 \varepsilon}\right)\right]
$$

Setting $b_{\phi}=b-\nabla \phi$, we obtain :

$$
\epsilon L_{\varepsilon}^{\prime} v=\epsilon^{2} \Delta_{g} v+\varepsilon<b_{\phi}, \nabla v>_{g}+v\left(\varepsilon\left(c+\frac{\Delta_{g} \phi}{2}\right)+\frac{<\nabla \phi, b>_{g}}{2}-\frac{\|\nabla \phi\|_{g}^{2}}{4}\right)
$$

If we set $v_{\varepsilon}=e^{-\frac{\phi}{2 \epsilon}} u_{\varepsilon}$, the equation

$$
\epsilon \Delta_{g} u_{\varepsilon}+<b, \nabla u_{\varepsilon}>+c u_{\varepsilon}=\lambda_{\varepsilon} u_{\varepsilon}
$$

becomes :

$$
\epsilon^{2} \Delta_{g} v_{\epsilon}+\epsilon<b_{\phi}, \nabla v_{\epsilon}>_{g}+c_{\epsilon} v_{\epsilon}=\epsilon \lambda_{\epsilon} v_{\epsilon} \text {, on } V
$$

where $c_{\epsilon}=\epsilon\left(c+\frac{\Delta \phi}{2}\right)+\frac{\left\langle\nabla \phi, b_{\phi}>_{g}\right.}{2}+\frac{\|\nabla \phi\|_{g}^{2}}{4}$.

We set $b_{\phi}=\Omega$. $c-\frac{\Delta \phi}{2}-\frac{d i v b_{\phi}}{2} \geq 0$. Taking $\varphi=\mathcal{L}$, a special Lyapunov function for $\Omega$ in the previous paragraph, we will prove that $\epsilon \lambda_{\epsilon}$ tends to the minimum of the function $\Psi_{\mathcal{L}}=\frac{(\nabla \mathcal{L})^{2}}{4}+\frac{(\Omega, \nabla \mathcal{L})}{2}$ when $\epsilon$ goes to zero.

Lemma 9 Under the assumptions of Theorem 7 on the vector field

$$
\lim _{\epsilon \rightarrow 0} \epsilon \lambda_{\epsilon}=0=\min _{V} \Psi_{\mathcal{L}}
$$

Proof: Multiplying equation (59) by $v_{\epsilon}$ and integrating on $\mathrm{V}$, we obtain:

$$
\int_{V}\left[\epsilon^{2}\left\|\nabla v_{\epsilon}\right\|_{g}^{2}+\left(-\frac{\epsilon \operatorname{div}\left(b_{\phi}\right)}{2}+c_{\epsilon}\right) v_{\epsilon}^{2}\right] d v o l_{g}=\epsilon \lambda_{\epsilon} \int_{V} v_{\epsilon}^{2} d v o l_{g}
$$


Normalize $\mathrm{v}_{\varepsilon}$ so that $\int_{V} v_{\epsilon}^{2} d v o l_{g}=1$. From equation (61), we obtain that $\lim _{\epsilon \rightarrow 0} \epsilon \lambda_{\epsilon} \geq$ $\min _{V} \Psi_{\phi}$. An upper bound of the function $\epsilon \lambda_{\epsilon}$, in the case when $\phi=\mathcal{L}$, will be computed using the following two results:

1) Let $S$ be a regular sub-domain of $V$ and $\lambda_{\epsilon}^{\prime}(S)$ be the first eigenvalue of $L_{\epsilon}^{\prime}$ on $S$ for the homogeneous Dirichlet problem. If there exists a positive number $A$ and a positive function $\psi$ on $S$, zero on the boundary of $S$, such that $L_{\epsilon}^{\prime} \psi \leq \epsilon A \psi$, then $\lambda_{\epsilon}^{\prime}(S) \leq \epsilon A$ (see [3])).

2) If $\lambda_{\epsilon}$ is the first eigenvalue of $L_{\epsilon}$ on $V$, then $\varepsilon \lambda_{\epsilon} \leq \lambda_{\epsilon}^{\prime}(S)$. Note that $\varepsilon \lambda_{\epsilon}$ is the first eigenvalue of $L_{\varepsilon}^{\prime}$ on $\mathrm{V}([3])$.

We proceed here with the proof in the case of a limit cycle and leave the case of a singular point, the easier one, to the reader. We construct a test function $\psi$ as follow. Let $\omega$ be a stable limit cycle of b. Let $\Gamma_{\delta}$ be the connected component containing $\omega$, of the set $\{P \in V \mid \mathcal{L}(P) \leq \mathcal{L}(\omega)+\delta\}$. For $\delta$ sufficiently small, $\Gamma_{\delta}$ is a regular sub-domain of $V$ and a neighborhood of $\omega$ not intersecting any other recurrent set of $b_{\mathcal{L}}$. For simplicity we set $b_{\mathcal{L}}=B$.

Define the function $\psi: \Gamma_{\delta}->\mathbb{R}$, as follow :

$$
\psi=e^{-\mathcal{L} /(2 \epsilon)}-e^{-\delta /(2 \epsilon)}
$$

Denoting by $\nabla$ the gradient with respect to g, we have:

$$
\begin{gathered}
\epsilon^{2} \Delta_{g} \psi=e^{-\mathcal{L} /(2 \epsilon)}\left[\frac{\varepsilon \Delta_{g} \mathcal{L}}{2}-\frac{\|\nabla \mathcal{L}\|_{g}^{2}}{4}\right] \\
L_{\epsilon}^{\prime}(\psi)=\epsilon c \psi-e^{-\delta /(2 \epsilon)}\left[\frac{\epsilon \Delta_{g} \mathcal{L}}{2}+\frac{<\nabla \mathcal{L}, \Omega>_{g}}{2}+\frac{\|\nabla \mathcal{L}\|_{g}^{2}}{4}\right]
\end{gathered}
$$

Let $R$ be a positive number,

$$
L_{\epsilon}^{\prime} \psi-\epsilon R \psi=e^{-\delta /(2 \epsilon)}\left[\epsilon(c-R)\left(e^{\frac{\delta-\mathcal{L}}{2 \epsilon}}-1\right)-\frac{\epsilon \Delta_{g} \mathcal{L}}{2}-\frac{<\nabla \mathcal{L}, B>_{g}}{2}-\frac{\|\nabla \mathcal{L}\|_{g}^{2}}{4}\right]
$$

Below we shall determine $R$ and $\delta$ so that $L_{\epsilon}^{\prime} \psi-\epsilon R \psi \leq 0$. Assume this done, using results 1 and 2 above, with $S=\Gamma_{\delta}$ we get that $\epsilon \lambda_{\epsilon} \leq \lambda_{\epsilon}^{\prime}(S) \leq \epsilon R$. Hence $\lim _{\epsilon \rightarrow 0} \epsilon \lambda_{\epsilon}=0$. We use Lemma 11 to determine $R$ and $\delta$. We get that up to terms of order four at least, using appendix I,

$$
L_{\epsilon}^{\prime} \phi-\epsilon R \phi=e^{-\delta /(2 \epsilon)}\left(\epsilon(c-R)\left(e^{\frac{\delta-\mathcal{L}}{2 \epsilon}}-1\right)-\epsilon \operatorname{tr}_{g} A-(1-\mu / 2)\|A x\|^{2}\right)
$$

We set $\delta=2 \alpha \epsilon$ where $\alpha$ will be determined later. There are two cases. If $\mathcal{L} \leq \alpha \epsilon$, then $e^{\frac{\delta-\mathcal{L}}{2 \epsilon}}-1 \geq e^{\frac{\alpha}{2}}-1$ and we can choose $\alpha$ so that $\left(e^{\frac{\alpha}{2}}-1\right)$ is arbitrarily large. $\|\bar{A} x\|^{2} \leq C \epsilon$ where $C>0$ depends only on the matrix A and $\alpha$. When $\epsilon$ converges to zero, for $R$ big enough and $\mathcal{L} \leq \alpha \epsilon$ :

$$
L_{\epsilon}^{\prime} \phi-\epsilon R \phi \leq \epsilon\left[(c-R)\left(e^{\frac{\alpha}{2}}-1\right)+\left|\operatorname{tr}_{g} A\right|\right] \leq 0
$$


When $\mathcal{L} \geq \alpha \epsilon$, then

$$
(c-R)\left(e^{\frac{\delta-L}{2 \epsilon}}-1\right) \leq 0 \text { for } \mathrm{R} \text { large enough }
$$

and:

$$
L_{\epsilon}^{\prime} \phi-\epsilon R \phi \leq \epsilon\left(\operatorname{tr}_{g} A-\left(1-\frac{\mu}{2}\right) a_{1} \alpha\right) .
$$

Because $\mathcal{L}(x) \geq \alpha \epsilon$, it implies that $\|A x\|^{2} \geq a_{1} \alpha \epsilon$, where $a_{1}$ depends only on the matrix $A$. Finally $L_{\epsilon}^{\prime} \phi-\epsilon R \phi \leq 0$ if $\frac{\operatorname{tr} A}{\left(1-\frac{\lambda}{2}\right) a_{1}} \leq \alpha$. Hence for $\mathrm{R}$ large enough, $L_{\epsilon}^{\prime} \phi-\epsilon R \phi \leq 0$ in $\Gamma_{\delta}$. Using the results of $\left([9)\right.$, we obtain that $\epsilon \lambda_{\epsilon} \leq \lambda_{\epsilon}\left(\Gamma_{\epsilon}\right) \leq \epsilon R$. This ends the proof of Lemma 9

Now we will prove that any weak limit of $v_{\epsilon}^{2} d v o l_{g} / \int_{V} v_{\epsilon}^{2} d v o l_{g}$ as $\epsilon$ goes to zero, concentrates on the limit sets of $b$.

Lemma 10 All weak limits of measures $v_{\epsilon}^{2} d v o l_{g} / \int_{V} v_{\epsilon}^{2} d v o l_{g}$ as $\epsilon$ goes to zero, are concentrated on the minimum set of the function $\Psi_{\mathcal{L}}$.

\section{Remark.}

By construction $\Psi_{\mathcal{L}}$ attains its minimum on the limit sets of $\Omega$ only and this minimum is zero (note that on a repulsive set, $\mathrm{L}$ is maximal, and $\Psi_{\mathcal{L}}$ is also zero).

\section{Proof of the Lemma 10.}

Proceeding as in the self-adjoint case, we multiply equation (59) by $v_{\epsilon} \phi$, where $\phi$ is an arbitrary test function. We obtain after integration by parts:

$$
\epsilon^{2} \int_{V}\left[\phi\left\|\nabla v_{\epsilon}\right\|_{g}^{2}+\left(c_{\epsilon} \phi-\epsilon^{2} \Delta \phi / 2-\epsilon \operatorname{div}(\Omega \phi)\right) v_{\epsilon}^{2}\right]=\epsilon \lambda_{\epsilon} \int_{V} \phi v_{\epsilon}^{2}
$$

$c_{\epsilon}$ converges to $\Psi_{\mathcal{L}}$ and $\epsilon \lambda_{\epsilon}$ to $0=\min _{V} \Psi_{\mathcal{L}}$ as $\epsilon$ goes to zero.

Let $\mu$ be a weak limit of the measures $v_{\epsilon}^{2} d v o l_{g}$ then

$$
\int_{V} \phi\left(\min _{V} \Psi_{\mathcal{L}}-\Psi_{\mathcal{L}}\right) d \mu=0
$$

Since equation (62) is true for any test function $\phi, d \mu=0$ on the open set where $\Psi_{\mathcal{L}} \neq$ $\min _{V} \Psi_{\mathcal{L}}$. This shows that the support of $\mu$ is contained in the limit set of $\Omega$. Let $S_{1}, \ldots S_{p}$ be the stationary points of $\Omega$ and let $\Gamma_{1}, \ldots \Gamma_{q}$ be the limit cycles. We have the decomposition:

$$
\mu=\sum_{j=1}^{p} \gamma_{k}^{2} \delta_{S_{k}}+\sum_{k=1}^{q} \mu_{k}
$$

where $\mu_{k}$ is a measure supported by $\Gamma_{k}$. 


\subsubsection{Remarks on the limit measures}

We end this section with two remarks. An interesting question is whether the repulsive sets can be charged by limit measures. Were it to hapen this is similar to the fact, wellknown in the large deviations theory, that the trajectories of a stochastic system can exit with a positive probablity the basin of attraction of an attractor of its drift. The boundary of the basin plays the role of the repulsive sets here. In other words a particle moving according to such a system can escape from the basin of attraction.

The second remark deals with the relations between the limit measures whose existence was shown above, and the measures, called equilibrium measures, which maximize the topological pressure $P$, defined in the introduction. More precisely $P$ is given by the formula:

$$
P=\sup _{\nu \in M}\left[h_{\nu}\left(F^{1}\right)+\int_{V}\left(c+\phi^{u}\right) d \nu\right]
$$

where $\mathrm{M}$ is the set of probablity measures invariant by the flow of the drift. A measure $\mu \in$ $M$ is an equilibrium measure if it maximizes $h_{\nu}\left(F^{1}\right)+\int_{V}\left(c+\phi^{u}\right) d \nu$. For more explanations $\operatorname{see}(22])$.

\subsection{Final Remark}

The characterization of the set of possible limit measure in the nonvariational case is studied in 14, where we prove that the concentration can occur along some submanifolds of the recurrent sets of a hyperbolic field b. In some cases, we are able to show that the measure is absolutely continuous with respect to the Hausdorff measure induced on the set. The order at which the Lyapunov function vanishes, in the neighborhood of the recurrents, play the role of a filter that allows the eigensequence to concentrate only along a subset where the topological pressure is achieved.

\section{Appendix 1}

In this appendix, we give the construction of Lyapunov functions for Morse-Smale vector field satisfying the condition $\frac{1}{4}\left(\|\nabla \mathcal{L}\|_{g}^{2}+2<\nabla \mathcal{L}, b>_{g}\right)>0$. We start by a local construction near the recurrent sets and then give a global construction on a compact Riemannian manifold.

\subsection{Local construction of Lyapunov functions}

Lemma 11 Given a Riemannian manifold $(V, g)$, of dimension $n$ and a vector field $b$ on $V$, for any hyperbolic stationary point of $b$ or hyperbolic periodic orbit there exists a local Lyapunov function $\mathcal{L}$ at that point or periodic orbit such that on the domain of definition of $\mathcal{L}$ outside the set of hyperbolic points and periodic orbits, $\Psi(L)=\frac{1}{4}\left(\|\nabla \mathcal{L}\|_{g}^{2}+2<\right.$ $\left.\nabla \mathcal{L}, b>_{g}\right)>0$. 
Since $\Omega$ is a Morse-Smale vector field, the limit sets are contained in the union of critical points and limit cycles. We refer to Kamin (see [17, 18, for the construction of $\mathcal{L}$ in the case of a critical attractive point of the field.

First we construct $\mathcal{L}$ near an attractive orientable periodic orbit $\Gamma$. Then the normal bundle $\mathcal{N}$ to $\Gamma$ is trivial. Denote by $\mathrm{T}$ the minimal period of $\Gamma$. Let $\mathrm{p}: \mathbb{R} \rightarrow V$, be a periodic trajectory of period $\mathrm{T}$, the image of which is $\Gamma$. We consider a Fermi coordinate system $\left(\theta, x_{1}, . ., x_{n-1}\right)$ in a neighborhood $\mathcal{U}$ of the cycle. $\theta$ is the cyclic coordinate and $\left(e_{1}(\theta), . ., e_{n-1}(\theta)\right)$ is the corresponding orthonormal frame field trivializing the normal bundle of $\Gamma$ i.e.dx ${ }^{i}\left(e_{j}\right)=\delta_{i j}$, the Kronecker symbol, $1 \leq i, j \leq n-1$. If $\xi$ is a point in the domain $\mathcal{U}$ of this coordinate system,

$$
\xi=\exp _{p(\theta(\xi))}\left[\sum_{i=1}^{n-1} x_{i}(\xi) e_{i}(\theta(\xi))\right]
$$

In these coordinates:

$$
g=\sum_{i, j=0}^{n-1} g_{i j} d x^{i} d x^{j},
$$

where we set $\mathrm{dx}_{0}=d \theta$. Along the cycle, $g_{i j}(\theta, 0)=\delta_{i j}, 1 \leq i, j \leq n-1, \mathrm{~g}_{0 j}(\theta, 0)=$ $g_{j 0}(\theta, 0)=0,1 \leq j \leq n-1$. The Christoffel symbols associated to the coordinates $\left(x_{1}, . ., x_{n-1}\right)$ are zero along $\Gamma: \Gamma_{i j}^{k}(\theta, 0)=0$, for $i, j, k \in\{1 . . n-1\}$. In a neighborhood of $\Gamma, g_{i j}(\theta, x)=\delta_{i j}+O(d(x))$. In this coordinates the equation $\frac{T \xi}{d t}=\Omega(\xi)$ can be written as follows:

$$
\begin{aligned}
& \dot{\theta}=1+O(d(x)) \\
& \dot{x}=B(\theta) x+O\left(d^{2}(x)\right)
\end{aligned}
$$

The solutions are given by

$$
\theta(t)=\theta(\xi(t)), x_{i}(t)=x_{i}(\xi(t)), 1 \leq i \leq n-1,
$$

where $\xi$ is trajectory of $b$ and $\mathrm{d}(\mathrm{x})$ is the distance from $\mathrm{x}$ to $\Gamma, B(\theta)$ is a $(\mathrm{n}-1) \mathrm{x}(\mathrm{n}-1)$ matrix-valued function.

Consider the solution $\mathrm{X}: \mathbb{R} \rightarrow G L[n-1 ; \mathbb{R}]$ of the matrix equation $\dot{X}=B(\theta) X$ such that $X(0)=I d_{n-1}$. Then there exist a (in general complex) matrix $\mathrm{D}$ and a matrix function $\mathrm{P}: \theta \in \mathbb{R} \rightarrow P(\theta) \in \mathrm{M}[(\mathrm{n}-1) \mathrm{x}(\mathrm{n}-1) ; \mathbb{C}]$, such that $X(\theta)=P(\theta) e^{\theta D}$ for all $\theta \in \mathbb{R}$. Because the orbit $\Gamma$ is hyperbolic and attractive, the real parts of the eigenvalues of $D$ are negative: the eigenvalues of $\mathrm{D}$ are exactly the characteristic multipliers of the orbit.

Denote by $\mathrm{D}^{*}$ the complex conjugate transpose of $\mathrm{D}$. Let $\mu$ be a strictly positive parameter. Consider the matrix Lyapunov equation in the unknown matrix A:

$$
A D+D^{*} A=-\mu A^{2} .
$$

It is well known that there exists a symmetric positive definite (n-1)x(n-1) matrix A solution of this equation, given by the formula:

$$
A^{-1}=\mu \int_{0}^{+\infty} e^{t D^{*}} e^{t D} d t
$$


We define a local Lyapunov function $\mathcal{L}: \mathcal{U} \rightarrow \mathbb{R}$ as follows: if $\xi \in \mathcal{U}$;

$$
\xi=\exp _{p(\theta(\xi)}\left[\sum_{k=1}^{n-1} x_{k}(\xi) e_{k}(\theta(\xi))\right]
$$

then: $\mathcal{L}=(A X, \bar{X})_{\mathbb{R}^{(n-1)}}$ where $\mathrm{X}=\mathrm{P}\left(\theta(\xi)^{-1}\left(\mathrm{x}_{1}(\xi), \ldots, \mathrm{x}_{(n-1)}(\xi)\right)\right.$ and the bar denotes the complex conjugate. Let $\xi: \mathrm{t} \in \mathbb{R}_{+} \rightarrow \xi(\mathrm{t}) \in \mathrm{V}$, be a positive semi- trajectory of b contained in $\mathcal{U}$ and let $\mathrm{X}(\mathrm{t})=\mathrm{P}(\theta(\xi))^{-1}\left(\mathrm{x}_{1}(\xi(t)), \ldots, \mathrm{x}_{(n-1)}(\xi(t))\right)$.

$$
\begin{gathered}
\frac{d X(t)}{d t}=B X(t)+O\left(\|X(t)\|^{2}\right) \\
\frac{d \mathcal{L}(\xi(t))}{d t}=\left(A \frac{d X(t)}{d t}, \overline{X(t)}\right)+\left(A X(t), \frac{\overline{d X(t)}}{d t}\right) \\
\left.\frac{d \mathcal{L}(\xi(t))}{d t}=\left(A D+D^{*} A\right) X(t), \overline{X(t)}\right)+O\left(\|X(t)\|^{3}\right) \\
\frac{d \mathcal{L}(\xi(t))}{d t}=-\lambda(A X(t), A \overline{X(t)})+O\left(\|X(t)\|^{3}\right)
\end{gathered}
$$

where for $\mathrm{U}, \mathrm{V} \in \mathbb{C}^{(n-1)}, \quad(\mathrm{U}, \mathrm{V})=\sum_{k=1}^{(n-1)} U_{k} V_{k}$. Hence $\mathrm{L}$ is decreasing along the trajectories of the field $b$ in a sufficiently small neighborhood of $\Gamma$.

$$
\begin{gathered}
(\nabla \mathcal{L}(\xi(t)), b(\xi(t)))=(A X(t), A \overline{X(t)})+O\left(\|X(t)\|^{3}\right) \\
\left(\nabla \mathcal{L}(\xi(t)), \nabla \mathcal{L}(\xi(t))=4(A X(t), A \overline{X(t)})+O\left(\|X(t)\|^{3}\right)\right.
\end{gathered}
$$

and

$$
\Delta \mathcal{L}=-2 \operatorname{tr}(A)
$$

Recall that $\Psi(L)=\frac{|\nabla L|^{2}}{4}+\frac{(\nabla L, \Omega)}{2}$ and thus

$$
\Psi()=(1-\lambda / 2)(A X(t), A \overline{X(t)})+O\left(\|X(t)\|^{3} .\right.
$$

For $1-\lambda / 2>0, \Psi(L)$ is strictly positive in the neighborhood of the cycle.

Assume now that we have a periodic orbit $\gamma$ of $\mathrm{b}$ with minimal period $\mathrm{T}$ which reverses the orientation. Let $\Pi: V \rightarrow V$ be the covering of $\mathrm{V}$ associated to the cyclic subgroup of $\pi_{1}(\mathrm{~V})$ generated by $2[\gamma]([\gamma]=$ homotopy class of $\gamma)$. This is a Galois covering space with group $\mathbb{Z}_{2}$. Denote by $i$ the nontrivial deck transformation of this covering. $\mathrm{g}$ and $\mathrm{b}$ have unique lifting to $V$ still denoted by g and b. $\Gamma=\Pi^{-1}(\gamma)$ is a periodic orbit of the lifting of $\mathrm{b}$ (i.e) b of minimal period $2 \mathrm{~T}$. We can apply the preceding construction to $\Gamma$ but with extra care here because of the deck involution $i$. $\mathrm{g}$ and $\mathrm{b}$ are invariant by $i$. We choose the neighborhood $\mathcal{U}$ invariant by $i$ and the Fermi coordinate system so that:

$$
\operatorname{Tie}_{k}(\theta)=e_{k}(\theta+T) \text { for } 0 \leq \mathrm{k} \leq(n-1)
$$


Then the previous construction produces a Lyapunov function $L$ for the lifting of b in a neighborhood of $\Gamma$. This function is invariant by the Galois group of the covering and hence can be pushed down by $\Pi$ to a Lyapunov function $L$ of $b$ in a neighborhood of $\gamma: \mathrm{L}=L \circ \Pi$.

For repulsive orbits the same construction applies changing b into - b. Finally the general case of a general hyperbolic periodic orbit $\Gamma$ can be easily handled by noticing that $\Gamma$ has a basis of open neighborhoods $\mathcal{U}$ such that $\mathcal{U}$ is diffeomorphic to the fiber product over $\Gamma$ of $\mathcal{U} \cap \mathrm{W}^{s}(\Gamma)$ and $\mathcal{U} \cap \mathrm{W}^{u}(\Gamma)$ and then "patching up" the Lyapunov functions constructed above for the restrictions of $b$ to the stable and unstable manifolds $\mathrm{W}^{s}(\Gamma)$, $\mathrm{W}^{u}(\Gamma)$ of $\Gamma$.

More precisely, due to the transversal intersection of the stable manifold with the unstable manifold at the hyperbolic set, a Lyapunov function is built in each set as follows: $N^{s}$ (resp. $N^{u}$ ) denotes the normal bundle of the stable (resp. unstable) manifold at $\Gamma$. Inside each normal fiber, a Lyapunov function is found. The coordinate system at a point $\xi \in \mathcal{U}$ is such that $\xi=\exp _{p(\theta(\xi)}\left[\sum_{k=1}^{n-1} x_{k}(\xi) e_{k}(\theta(\xi))\right]=\exp _{p(\theta(\xi)}\left[X_{s}+X_{u}\right]$ then as in the first part of the lemma, we can defined find two matrices $A_{u}$ and $A_{s}$ solving the matrix Lyapunov equation in each subspace $N^{u}$ and $N^{s}$ respectively. If we denote $n_{u}=\operatorname{dim} N^{u}$ and, $n_{s}=\operatorname{dim} N^{s}$, then $n_{u}+n_{s}-1=n$ and we can now define the Lyapunov function $\mathcal{L}$ by : $\mathcal{L}(\xi)=\left(A_{s} Y_{s}, \bar{Y}_{s}\right)_{\mathbb{R}^{\left(n_{s}\right)}}-\left(A_{u} Y_{u}, \bar{Y}_{u}\right)_{\mathbb{R}^{\left(n_{u}\right)}}$ where $\mathrm{Y}_{u}=P_{u}\left(\theta(\xi)^{-1}\left(\mathrm{x}_{1}(\xi), \ldots, \mathrm{x}_{\left(n_{u}\right)}(\xi)\right)\right.$ and $\mathrm{Y}_{s}=P_{s}\left(\theta(\xi)^{-1}\left(\mathrm{x}_{n_{u}+1}(\xi), \ldots, \mathrm{x}_{(n-1)}(\xi)\right)\right.$. Here $P_{u}$ and $P_{s}$ are two matrices defined as in the first part of the Lemma for the solution of the matrix equation in each normal bundle. From the construction, it follows that $\mathcal{L}$ is positive and decays along the trajectory of the vector field.

We can repeat this type of construction locally in a any neighborhood of a limit set of the field. Indeed since the limit set is hyperbolic, the construction of the function $\mathcal{L}$ is possible on each attractive or repulsive fiber and due to the transversal intersection we can match the construction and the function $\mathcal{L}$ vanishes only on the limit set in these neighborhoods. Outside the limit set, there is a large choice of extensions of the function $\mathcal{L}$.

\subsection{Global construction of Lyapunov functions}

In this section we prove the existence of a global Lyapunov function, assuming the existence of a local one (proved in the last section).

$M$ is a $C^{\infty}$ compact Riemannian manifold and $\phi_{t}$, a Morse-Smale flow. $F$ is the vector field on $\mathrm{V}$, generator of the flow. Let us denote by $\Omega$ the limit set of the flow. $\Omega$ is endowed with a partial order as follows: if $\omega_{1}, \omega_{2}$ belong to $\Omega, \omega_{1} \succ \omega_{2}$ if there exists a trajectory the $\alpha$ - limit set of which is $\omega_{1}$ and the $\omega$-limit set is $\omega_{2}$. This partial order determines a filtration of $\Omega: \Omega=\Omega_{0} \supset \Omega_{1} \supset \ldots \supset \Omega_{m}$ as follows: Let $\Omega_{0, \max }$ be the set of all maximal elements of $\Omega$. Set $\Omega_{1}=\Omega-\Omega_{0, \max }$. Let $\Omega_{1, \max }$ be the set of the maximal elements of $\Omega_{1}$. Set $\Omega_{2}=\Omega_{1}-\Omega_{1, \max }$ and so on. When $\Omega_{n}$ has been defined, let $\Omega_{n, \max }$ be the set of all maximal elements of $\Omega_{n}$. Set $\Omega_{n+1}=\Omega_{n}-\Omega_{n, \max }$. This filtration ends at a certain $\mathrm{m}$. 
$\Omega_{m}$ is the set of all minimal elements of $\Omega$.

\subsubsection{Local Lyapunov functions}

A strict Lyapunov function for $F$ on an open subset $O$ of $M$ is a $C^{\infty}$ function $\mathcal{L}: O \rightarrow$ $M$ such that $d \mathcal{L}(F)<0$ in $O-\Omega$. For $\omega \in \Omega$ there exists two relatively compact neighborhoods $O_{\omega}$ and $\tilde{O}_{\omega}$ of $\omega$ in $M_{n}$ such that the closure $\bar{O}_{\omega}$ of $O_{\omega}$ in M, is contained in $\tilde{O}_{\omega}$ and a strict Lyapunov function $\mathcal{L}_{\omega}: \tilde{O}_{\omega} \rightarrow \mathbb{R}$ such that:

(i) $\tilde{O}_{\omega} \cap \tilde{O}_{\omega^{\prime}}=\varnothing$, for $\omega, \omega^{\prime} \in \Omega, \omega \neq \omega^{\prime}$.

(ii) The boundary $\partial O_{\omega}$ is the union of $\partial_{s} O_{\omega} \cup \partial_{u} O_{\omega} \cup \partial_{l} O_{\omega}$ where the three components are compact codimension 1 submanifolds with boundaries $\partial \partial_{s} O_{\phi}, \partial \partial_{u} O_{\omega}, \partial \partial_{l} O_{\omega}$ such that $\partial \partial_{s} O_{\omega} \cup \partial \partial_{u} O_{\omega}=\partial \partial_{l} O_{\omega}$ and $\partial_{s} O_{\omega} \cap \partial_{u} O_{\omega}=\emptyset$.

(iii) $\partial_{s} O_{\omega}$ and $\partial_{u} O_{\omega}$ are transversal to the flow and $\partial_{l} O_{\omega}$ is foliated by arcs of trajectories of the flow $\phi_{t}$ linking $\partial_{s} O_{\omega}$ to $\partial_{u} O_{\omega}$.

(iv) $L_{\omega}$ is constant on $\partial_{s} O_{\omega}$ and $\partial_{u} O_{\phi}$.

(v) $\partial_{s} O_{\omega} \cap W^{s}(\omega)$ (resp. $\partial_{u} O_{\omega} \cap W^{u}(\omega)$ ) is a compact $C^{\infty}$ sub-manifold contained in $W^{s}(\omega)$ (resp. $\left.W^{s}(\omega)\right)$ which is a section of the restriction of the flow to $W^{s}(\omega)$ (resp. $W^{u}(\omega)$ ). If $\omega$ is maximal in $\Omega, \partial_{l} O_{\omega}=\emptyset=\partial_{s} O_{\omega}$. If $\omega$ is minimal in $\Omega, \partial_{l} O_{\omega}=\emptyset=\partial_{u} O_{\omega}$.

\subsubsection{Construction of the global Lyapunov function}

The construction is inductive. To fix the ideas, we can always assume that, if $\omega$ is neither maximal nor minimal, $\mathcal{L}_{\omega}\left(\partial_{s} O_{\omega}\right)=1, \mathcal{L}_{\omega}\left(\partial_{u} O_{\omega}\right)=-1 \mathcal{L}_{\omega}(\omega)=0$ and $\mathcal{L}_{\omega}\left(\bar{O}_{\omega}\right)=[-1,1]$.

In the case $\omega$ is maximal, we can assume that $O_{\omega}$ is an open ball whose boundary $\partial_{u} O_{\omega}\left\{\mathcal{L}_{\omega}(\omega)=0\right\}$ is a sphere that $\mathcal{L}_{\omega}(\omega)=-1$ and $\mathcal{L}_{\omega}\left(\bar{O}_{\omega}\right)=[-1,0]$.

In the case $\omega$ is minimal, we can assume that $O_{\omega}$ is an open ball whose boundary $\partial_{s} O_{\omega}$ is a sphere $\left\{\mathcal{L}_{\omega}(\omega)=0\right\}$, that $\mathcal{L}_{\omega}(\omega)=1$ and $\mathcal{L}_{\omega}\left(\bar{O}_{\omega}\right)=[0,1]$.

The construction starts as follows: Let $M_{0}=\left\{\cup \bar{O}_{\omega} \mid \omega \in \Omega_{0}^{\max }\right\} . \quad M_{0}$ is a compact manifold with boundary $\left\{\cup \partial_{u} O_{\omega} \mid \omega \in \Omega_{0}^{\max }\right\}$. Define $\mathcal{L}_{0}: M_{0} \rightarrow \mathbb{R}$, as follows $\mathcal{L}_{0 \mid \bar{O}_{\omega}}=\mathcal{L}_{\omega}$. It is clear that

(i) $M_{0} \cap \cup\left\{\tilde{O}_{\omega}, \omega \in \Omega_{1}\right\}=\emptyset$

(ii) $M_{0}$ is a sub-manifold of codimension 0 with boundary $\partial M_{0}$.

(iii) $\mathcal{L}_{0}$ : is a strict Lyapunov function on $M_{0}$ and $\mathcal{L}_{0}\left(\partial M_{0}\right)=-1$

Assume now that we have constructed a sub-manifold $M_{n}$ of codimension 0 in $M$ with boundary $\partial M_{n}$, and a strict Lyapunov function $\mathcal{L}_{n}: M_{n} \rightarrow \mathbb{R}$, such that

(i) $M_{n}$ contains $\cup_{k=0}^{n} \Omega_{k}^{\max }$ in its interior and $M-M_{n} \supset \cup\left\{\bar{O}_{\omega} \mid \omega \in \Omega_{n+1}\right\}$

(ii) $L_{n}$ is a constant on $\partial M_{n}$ and equal to $c_{n}$, say.

For each $\omega \in \Omega_{n+1}^{\max }$ let $V_{\omega}=\partial M_{n} \cap \cup\left\{\phi_{-t}\left(\partial_{s} O_{\omega}\right) \mid t \geq 0\right\}$. Then $V_{\omega}$ is a compact sub-manifold of $\partial M_{n}$ of codimension 0 with boundary $\partial V_{\omega}=\partial M_{n} \cap\left\{\phi_{-t}\left(\partial \partial_{s} O_{\omega}\right) \mid t \geq 0\right\}$ in $\partial M_{n}$.

Also

(i) $V_{\omega} \cap V_{\omega^{\prime}}=\emptyset$ for $\omega, \omega^{\prime} \in \Omega_{n+1}^{\max }$ and $\omega \neq \omega^{\prime}$

(ii) $V_{\omega}$ contains the manifold $\partial M_{n} \cup W_{\omega}^{s}$ in its interior. 
There is for each $\omega \in \Omega_{n+1}^{\max }$ a $C^{\infty}$ function $\left.T_{s, \omega}: V_{\omega} \rightarrow\right] 0,+\infty$ [ such that for any $x \in V_{\omega}, \phi(t, x) \notin \bar{O}_{\omega}, 0 \leq t \leq T_{s, \omega}(x)$ and $\phi\left(T_{s, \omega}(x), x\right) \in \partial_{s} O_{\Omega}$.

Let $\epsilon$ be a small positive number $\epsilon<i n f_{x \in V_{\omega}} T_{s, \omega}(x)$ and let $D_{\omega}^{1} \subset \mathbb{R} \times V_{\omega}$ be the subset $\left\{(t, x) \mid x \in V_{\omega},-\epsilon \leq t \leq T_{s, \omega}(x)\right\}$. $\phi$ maps $D_{\omega}^{1}$ diffeomorphically into $M_{n}$. $D_{\omega}^{1}$ and $\phi\left(D_{\omega}^{1}\right)$ are manifolds with corners.

We can choose a $C^{\infty}$ function $\left.\hat{c}_{\omega}: D_{\omega}^{1} \rightarrow\right] 0,+\infty[$ such that

(i) if $x \in V_{\omega}$ and $-\epsilon \leq t \leq 0, \hat{c}_{\omega}(t, x)=-\frac{d}{d t} \mathcal{L}_{n}\left(\phi_{t}\right)$

(ii) if $x \in V_{\omega}$ and $T_{s, \omega}(x)-\epsilon \leq t \leq T_{s, \omega}(x), \hat{c}_{\omega}(t, x)=-\frac{d}{d t} \mathcal{L}_{\omega}\left(\phi_{t}\right) . \mathcal{L}_{\omega}$ is defined on a neighborhood of $\partial_{s} O_{\Omega}$.

Let

$$
\Gamma=\sup _{\omega \in \Omega_{n+1}^{\max }, x \in V_{\omega}} \int_{0}^{T_{s, \omega}(x)} \hat{c}_{\omega}(t, x) d t
$$

Define now a function $\left.\lambda_{\omega}: V_{\omega} \rightarrow\right] 0,+\infty\left[\right.$ by the formula $\lambda_{\omega}(x)=\frac{T_{s, \omega}(x)-\epsilon}{\Gamma-\int_{0}^{T_{s, \omega}(x)} \hat{c}_{\omega}(t, x)} d t$ It is a $C^{\infty}$ function.

Let $\mathrm{u}$ be a $C^{\infty}$ function $\left.\mathbb{R} \rightarrow\right] 0,+\infty[$ such that $\operatorname{supp} u \in] 0,1\left[\right.$ and $\int_{0}^{1} u=1$. Let us define the function $\left.a_{\omega}: D_{\omega}^{1} \rightarrow\right] 0,+\infty\left[\right.$, as follows $a_{\omega}(t, x)=\lambda_{\omega}(x) u\left(\frac{t}{T_{s, \omega}(x)-\epsilon}\right)$. It is a $C^{\infty}$ function. Define $\left.c_{\omega}: D_{\omega}^{1} \rightarrow\right] 0,+\infty\left[\right.$ as the sum $\hat{c}_{\omega}+a_{\omega}$ then

(i) $\int_{0}^{T_{s, \omega}(x)} c_{\omega}(t, x) d t=\Gamma$

(ii) $c_{\omega}(t, x)=-\frac{d}{d t} \mathcal{L}_{n}\left(\phi_{t}(x)\right)$ for all $x \in V_{\omega}$ and $-\epsilon \leq t \leq 0$.

(iii) $c_{\omega}(t, x)=-\frac{d}{d t} \mathcal{L}_{\omega}\left(\phi_{t}(x)\right)$ for all $x \in V_{\omega}$ and $T_{s, \omega}(x)-\epsilon \leq t \leq T_{s, \omega}(x)$.

(iii) implies that for all $x \in V_{\omega}$ all t such that $T_{s, \omega}(x)-\epsilon \leq t \leq T_{s, \omega}(x)$,

$$
\begin{aligned}
& \int_{0}^{t} c_{\omega}(s, x) d s=\int_{0}^{T_{s, \omega}(x)} c_{\omega}(t, x) d t+\int_{T_{s, \omega}(x)}^{t} c_{\omega}(t, x) d t=\Gamma_{\omega}-\int_{T_{s, \omega}(x)}^{t} \frac{d}{d t} \mathcal{L}_{\omega}\left(\phi_{t}(x)\right) \\
& =\Gamma_{\omega}+\mathcal{L}_{\omega}\left(\phi_{T_{s, \omega}}(x), x\right)-\mathcal{L}_{\omega}\left(\phi_{t}(x), x\right)=\Gamma_{\omega}+1-\mathcal{L}_{\omega}\left(\phi_{t}(x), x\right)
\end{aligned}
$$

For each $\omega \in \Omega_{n+1}^{\max }$ let $U_{\omega}=\phi\left(D_{\omega}^{1}\right) \cup \bar{O}_{\omega}$. Define a function $\widehat{\mathcal{L}}_{\omega}: U_{\omega} \rightarrow \mathbb{R}$ as follows: if $y \in \phi\left(D_{\omega}^{1}\right), y=\phi_{t}(x),-\epsilon \leq t \leq T_{s, \omega}(x), \widehat{\mathcal{L}}_{\omega}(y)=c_{n}-\int_{0}^{t} c_{\omega}(s, x) d s$. If $y \in \bar{O}_{\omega}$, $\widehat{\mathcal{L}}_{\omega}(y)=c_{n}-\Gamma_{\omega}-1+\mathcal{L}_{\omega}(y)$ It is easy to see that $\widehat{\mathcal{L}}_{\omega}$ is a strict Lyapunov function on $U_{\omega}$ and $\widehat{\mathcal{L}}_{\omega}\left(V_{\omega}\right)=c_{n}$.

We will now proceed with the construction of $M$. For each $\omega \in \Omega_{n+1}^{\max }$, let $C V_{\omega}$ be a collar for $\partial V_{\omega}$ in $V_{\omega}$ such that $W^{s}\left(V_{\omega}\right) \cap \partial M_{n} \subset V_{\omega}-\overline{C V_{\omega}}$. There exists a $C^{\infty}$ function $\left.T_{u, \omega}: C V_{\omega} \rightarrow\right] 0,+\infty\left[\right.$ such that $\phi(t, x) \notin \partial_{u} O_{\omega}$ if $-\epsilon \leq t<T_{u, \omega}$ and $\phi\left(T_{u, \omega}(x), x\right) \in \partial_{u} O_{\omega}$. Let

$$
N_{n}=\left(\partial M_{n}-\cup\left\{V_{\omega}, \omega \in \Omega_{n+1}^{\max }\right\}\right) \cup\left\{C V_{\omega} \mid w \in \Omega_{n+1}^{\max }\right\}
$$

$N_{n}$ is a submanifold of codimension 0 with boundary in $\partial M_{n}$. It is easy to construct a $C^{\infty}$ function $\left.T_{u}: N_{n} \rightarrow\right] 0,+\infty\left[\right.$ such that $T_{u}=T_{u, \omega}$ for any $\omega \in \Omega_{n+1}^{\max }$. Let $D^{u}=\{(t, x), x \in$ $\left.N_{n} 0 \leq t \leq T_{u}(x)\right\}$. $\phi$ maps $D^{u}$ diffeomorphically on a manifold with corners of dimension 
0 in $M$. Then we can construct a $C^{\infty}$ function $\left.\tilde{c}: N_{n} \rightarrow\right] 0,+\infty[$ such that:

$$
\tilde{c}(t, x)=-\frac{d \widehat{\mathcal{L}}_{\omega}}{d t}
$$

Let $\left.\tilde{\gamma}: N_{n} \rightarrow\right] 0,+\infty\left[\right.$ be the function $\tilde{\gamma}(x)=\int_{0}^{T_{u}(x)} \tilde{c}(t, x) d t$. $\tilde{\gamma}$ is a $C^{\infty}$ function. We define $\left.c: N_{n} \rightarrow\right] 0,+\infty[$ as follows:

$$
c(t, x)=\frac{\tilde{c}(t, x)}{\tilde{\gamma}(x)}(\Gamma+2)
$$

then $c$ is a $C^{\infty}$ function and $\int_{0}^{T_{u}(x)} c(t, x)=\Gamma+2$. If $\omega \in \Omega_{n+1}^{\max }$ and $x \in C V_{\omega}$,

$$
\begin{gathered}
\tilde{\gamma}(x)=\int_{0}^{T_{u}(x)} c(t, x)=\int_{0}^{T_{u}(x)}-\frac{d \widehat{\mathcal{L}}_{\omega}\left(\phi_{t}(x)\right)}{d t}=\widehat{\mathcal{L}}_{\omega}(x)-\widehat{\mathcal{L}}_{\omega}\left(\phi_{T_{u}}(x)\right) \\
\tilde{\gamma}(x)=c_{n}-\left(c_{n}-1+\Gamma+\widehat{\mathcal{L}}_{\omega}\left(\phi_{t T_{u}}(x)\right)=\Gamma+2\right.
\end{gathered}
$$

Hence for $\omega \in \Omega_{n+1}^{\max }, c \in C V_{\omega},-\epsilon \leq t \leq T_{u}(x), c(t, x)=\tilde{c}(t, x)=-\frac{d \widehat{\mathcal{L}}_{\omega}\left(\phi_{t}(x)\right)}{d t}$.

Let

$$
M_{n+1}=M_{n} \cup \phi\left(D^{u}\right) \cup\left\{\cup U_{\omega} \mid \omega \in \Omega_{n+1}^{\max }\right\} .
$$

Define $\mathcal{L}_{n+1}: M_{n+1} \rightarrow \mathbb{R}$ as follows: if $y \in M_{n}, \mathcal{L}_{n+1}(y)=\mathcal{L}_{n}(y)$ if $y \in D^{u}, y=\phi_{t}(x), x \in$ $N_{\omega}, \mathcal{L}_{n+1}(y)=c_{n}-\int_{0}^{t} c(\tau, x) d \tau$ and if $y \in U_{\omega}, \omega \in \Omega_{n+1}^{\max }, \mathcal{L}_{n+1}(y)=\widehat{\mathcal{L}}_{\omega}(y)$.

This function is well defined and $C^{\infty}$. If $y \in \phi\left(D^{u}\right) \cap_{\omega \in \Omega_{n+1}^{\max }} \cup U_{\omega}$, then $y=\phi_{t}(x), x \in$ $C V_{\omega}$ for $0 \leq t \leq T_{u}(x)=T_{u, \omega}(x)$, but then

$$
c_{n}-\int_{0}^{t} c(\tau, x) d \tau=c_{n}+\int_{0}^{t} \frac{d \mathcal{L}_{\omega}\left(\phi_{v}(x)\right)}{d v}=c_{n}+\widehat{\mathcal{L}}_{\omega}\left(\phi_{t}(x)\right)-\widehat{\mathcal{L}}_{\omega}(x)=\widehat{\mathcal{L}}_{\omega}(y)
$$

By construction, $\mathcal{L}_{n+1}$ is a strict Lyapunov function on $M_{n+1} . M_{n+1}$ is a manifold of codimension 0 in $M$ with boundary $\partial M_{n}=\cup\left\{\partial_{u} O_{\omega} \mid \omega \in \Omega_{n+1}^{\max }\right\} \cup \tilde{N}$ where $\tilde{N}=$ $\left\{\phi\left(T_{u}(x), x\right), x \in N_{n}\right\}$. If $y \in \partial_{u} O_{\omega} \cap \tilde{N}$ then $y=\phi\left(T_{u}(x), x\right)$, for some $x \in C V_{\omega} \cdot T_{u}(x)=$ $T_{u, \omega}(x)$. We have $\partial M_{n+1}=\mathcal{L}_{n+1}^{-1}\left(c_{n+1}\right)$, where $c_{n+1}=c_{n+1}-\Gamma-2$.

Finally, we remark that $b \mathrm{MS}$ is equivalent to $\Omega \mathrm{MS}$, when

$$
b=\Omega+\nabla \mathcal{L} .
$$

\subsection{Appendix 2}

In this appendix we are going to provide the estimates needed in $\left(^{*}\right)$. On the Riemannian manifold $(\mathrm{V}, \mathrm{g})$ consider an operator of the form:

$$
L=\varepsilon \Delta_{g}+\varepsilon \theta(b)+\left(\psi+\sqrt{\varepsilon} c_{\varepsilon}\right),
$$


where $\psi$ is a non negative function on $\mathrm{V}$ and $\mathrm{c}_{\varepsilon}, \varepsilon \in[0,1]$, is an arbitrary function on $\mathrm{V}$, parametrized by $\varepsilon$, smooth in all the variables .

We assume that we have a family $\left.\left.\left\{\mathrm{u}_{\varepsilon} \mid \varepsilon \in\right] 0,1\right]\right\}$ of smooth functions on $\mathrm{V}$ such that: $\mathrm{u}_{\varepsilon} \geq 0$ for all $\varepsilon$ and

$$
\varepsilon \Delta_{g} u_{\varepsilon}+\varepsilon \theta(b) u_{\varepsilon}+\left(\psi+\sqrt{\varepsilon} c_{\varepsilon}\right) u_{\varepsilon}=0
$$

Then we can state a lemma:

Lemma 12 There exists constants $C, \varepsilon_{0}>0$, depending only on $g, b, \psi$, and $\max _{[0,1] \times V}\left|c_{\varepsilon}\right|$ (but independent of $\varepsilon$ !) and a universal mapping $\gamma: \mathbb{N}_{+} \longrightarrow \mathbb{N}_{+}$, such that for each integer $n \in \mathbb{N}^{+}$, for all $x \in V_{\psi}(=\{z \| z \in V, \psi(z) \neq 0\})$, all $\left.\left.\varepsilon \in\right] 0, \varepsilon_{0}\right]$ :

$$
u_{\varepsilon}(x) \leq\left(\max _{V} u_{\varepsilon}\right) \frac{\gamma(n)(C \varepsilon)^{\frac{n}{3}}}{\psi^{n}}
$$

It follows that at any $x \in V_{\psi}, u_{\varepsilon}(x)$ tends to zero faster than any power of $\varepsilon$ and the convergence is uniform on any compact in $V_{\psi}$.

Proof.

The proof will use be an induction on $\mathrm{n}$. Given any smooth functions $\mathrm{f}, \mathrm{h}$ on $\mathrm{V}$ we have:

$$
\begin{gathered}
f\left[\varepsilon \Delta_{g} h+\varepsilon \theta(b) h\right]=\varepsilon \Delta_{g}(f h)+\varepsilon \theta(\widehat{b})(f h)-f h\left[\varepsilon \frac{\Delta_{g} f}{f}+2 \varepsilon \frac{\|\nabla f\|_{g}^{2}}{f^{2}}+\varepsilon \frac{\theta(b) f}{f}\right] \\
f\left[\varepsilon \Delta_{g} h+\varepsilon \theta(b) h\right]=\varepsilon \Delta_{g}(f h)+\varepsilon \theta(\widehat{b})(f h)-f h \varepsilon\left[\frac{\Delta_{g} f}{f}+\frac{\theta(\widehat{b}) f}{f}\right] \\
\text { on } \mathrm{V}_{f},
\end{gathered}
$$

where $\widehat{b}=b+2 \frac{\nabla f}{f}, V_{f}=\{x \| \mathrm{x} \in V, f(x) \neq 0\}$. For each $\mathrm{n} \in \mathbb{Z}_{+}$, set $\mathrm{v}_{n}=\psi^{n} u_{\varepsilon}$. Write:

$$
\begin{gathered}
\psi^{n}\left[\varepsilon \Delta_{g} u_{\varepsilon}+\varepsilon \theta(b) u_{\varepsilon}+(\psi+\sqrt{\varepsilon} c) u_{\varepsilon}\right]=\frac{\psi^{n+1}}{\psi} \varepsilon\left[\Delta_{g} u_{\varepsilon}+\theta(b) u_{\varepsilon}\right]+(\psi+\sqrt{\varepsilon} c) u_{\varepsilon} \psi^{n}, \\
\text { on } \mathrm{V}_{\psi} .
\end{gathered}
$$

Applying the formula(72) to (174) taking $\mathrm{f}=\psi^{n+1}$ and $\mathrm{h}=\mathrm{u}_{\varepsilon}$, we get, for $\mathrm{n} \geq 2$ :

$$
\begin{gathered}
\left.\frac{1}{\psi} \varepsilon\left[\Delta_{g} v_{n+1}+\theta(\widehat{b}) v_{n+1}\right]+v_{n+1}+\sqrt{\varepsilon} c_{\varepsilon} v_{n}-(n+1) \varepsilon[\theta(\widehat{b}) \psi)+\Delta_{g} \psi\right] v_{n-1} \\
-(n+1)(n+2) \varepsilon\|\nabla \psi\|_{g}^{2} v_{n-2}=0 .
\end{gathered}
$$

Let $\mathrm{M}_{n}=\max _{V} \mathrm{v}_{n}$. Note that $\mathrm{M}_{0}=\max _{V} u_{\varepsilon}$. Choose a point $\mathrm{P} \in \mathrm{V}$ such that $\mathrm{M}_{n+1}=\mathrm{v}_{n+1}(\mathrm{P})$. Note that $\psi(\mathrm{P}) \neq 0$. Evaluate the equation(75) at $\mathrm{P}$.

$$
\varepsilon\left[\Delta_{g} v_{n+1}(P)+\theta(\widehat{b}) v_{n+1}\right](P)=\varepsilon \Delta_{g} v_{n+1}(P) \geq 0
$$




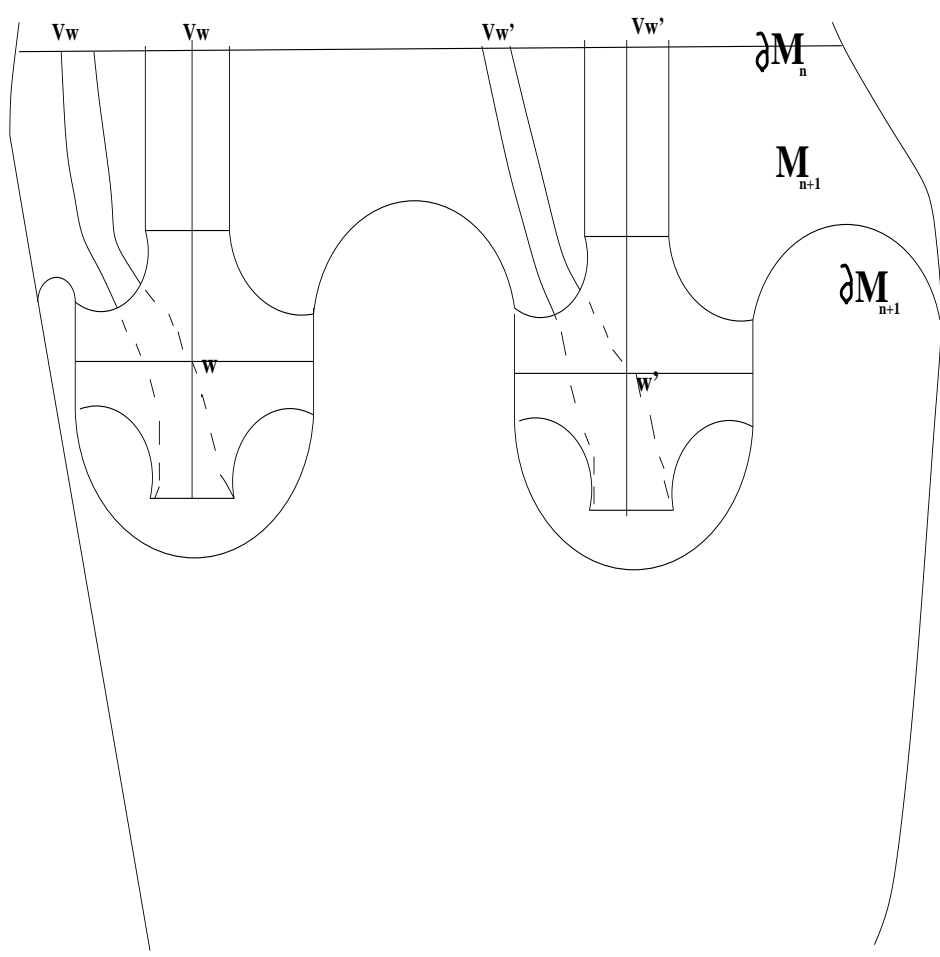

Figure 1: Construction by induction of the global Lyapunov function

For $\mathrm{n} \geq 2$ :

$$
\begin{gathered}
\left.v_{n+1}(P)+\sqrt{\varepsilon} c_{\varepsilon}(P) v_{n}(P)-(n+1) \varepsilon[\theta(\widehat{b}) \psi)+\Delta_{g} \psi\right](P) v_{n-1}(P) \\
-(n+1)(n+2) \varepsilon\|\nabla \psi\|_{g}^{2}(P) v_{n-2}(P) \leq 0
\end{gathered}
$$

The relation(76) implies the inequality:

$$
M_{n+1} \leq \sqrt{C \varepsilon} M_{n}+(C \varepsilon)(n+1) M_{n-1}+(C \varepsilon)(n+1)(n+2) M_{n-2}, \text { for } \mathrm{n} \geq 2,
$$

where :

$$
C=\max \left(\sup _{V \times[0,1]}\left|c_{\varepsilon}\right|, \sup _{V}\left[\sqrt{|\theta(b) \psi|+\left|\Delta_{g} \psi\right|},|| \nabla \psi \mid \|_{g}\right]\right) .
$$

The relation (77) implies that:

$$
\frac{M_{n+1}}{(n+2) !} \leq \sqrt{C \varepsilon} \frac{M_{n}}{(n+1) !}+C \varepsilon \frac{M_{n-1}}{n !}+C \varepsilon \frac{M_{n-2}}{(n-1) !} \text {, for } \mathrm{n} \geq 2
$$

Clearly $\mathrm{M}_{n} \leq \max \left(1, \max _{V} \psi, \max _{V} \psi^{2}\right) \mathrm{M}_{0}$, if $\mathrm{n}=0,1,2$. The lemma follows easily from this remark and the reccurence relation(78). 


\section{References}

[1] T. Aubin- Some Nonlinear Problems in Riemannian Geometry; Springer, 1998

[2] A. Devinatz- R.Ellis-A. Friedman- The Asymptotic behavior of the First Real Eigenvalue of Second Order Elliptic Operators with a Small Parameter in the Highest Derivative, II Indiana Univ. Math. J. 23, No. 11, 1974 p991-1011.

[3] A. Devinatz- A. Friedman- Asymptotic Behavior of the Principal Eigenfunction for a Singularly Perturbed Dirichlet Problem Indiana Univ. Math. J. 27, No. 1, 1978 p143-157.

[4] A. Devinatz-A. Friedman-The Asymptotic behavior of the solution of a singular perturbed Dirichlet problem Indiana Univ. Math. J. 27, No. 3, 1978 p527-537.

[5] S. Dobrokotov-V. Kolokol'tsov-V. Maslov-, splitting of the low energy levels of the Schrödinger equation and the asymptotic behavior of the fundamental solution of the equation $h u_{t}=\frac{1}{2} h^{2} \Delta u-v(x) u$. Theor. and Math. Phys., 87, 561-99 (1991).

[6] M. Donsker S. Varadhan On a variational formula for the principal eigenvalue for the operators with maximum principle, Proc. Nat. Acad. Sci. USA, 72, 1975, p780-783.

[7] W. Fleming- Controlled Markov Processes, 1986, Pisa.

[8] M. Freidlin-A. Wentzell-Random Perturbations of Dynamical Systems, Springer-Verlag, 1984.

[9] A. Friedman-The Asymptotic behavior of the First Real Eigenvalue of Second Order Elliptic Operators with a Small Parameter in the Highest Derivative, Indiana Univ. Math. J. 22, No. 10, 1973 p1005-1015.

[10] D. Gilbarg - N. Trudinger -Elliptic Partial differentiable equations of second order; Springer-Verlag 1983, second edition.

[11] B. HelfFer-J. SJÖstRAND-Multiple Wells in the semi-classical limit I. Comm. in P.D.E, 9(4), 1984 p337-408.

[12] B. HELFFER- Semiclassical analysis for the Schrodinger operator and applications, Springer Lectures Notes in Math. 1336, 1988.

[13] D. Holcman, I. Kupka- Singular Perturbation and first order PDE on manifolds. C. R. Acad. Sci. Paris Sér. I Math. 333 (2001), no. 5, 465-470.

[14] D. Holcman, I. KupKa- Semi-classical limit of the first eigenfunction and concentration on the recurent sets of a dynamical system, pre-print. 
[15] D.Holcman, A. Marchevska, Z. Schuss- The survival probability of diffusion with killing, pre-print.

[16] C. Holland -Stochastically perturbated limit cycles, J. Appl. Prob. 15, 311-320 1978.

[17] S. KAmin-Exponential descent of solutions of elliptic singular perturbation problems, Comm P.D.E, 9(2) 1984p197-213.

[18] S. KAmin- Singular perturbation problems and Hamilton-Jacobi Integral Equations and Operator Theory, Vol. 9, 1986 p95-105.

[19] Y. KIFER-Stochastic stability of the topological pressure. J. Analyse Math. 38 (1980), $255-286$.

[20] Y. KIFER-On the principal eigenvalue in a singular perturbation problem with hyperbolic limit points and circles. J. Differential Equations 37 (1980), no. 1, 108-139.

[21] Y. KIfER-- Random Perturbations of Dynamical Systems, Birkauser, 1988.

[22] Y. KIFER-Principal eigenvalues, topological pressure, and stochastic stability of equilibrium states. Israel J. Math. 70 (1990), no. 1, 1-47.

[23] M. Krasnoselskil- Positive solutions of Operator Equations, Noordhoff, Gromingen, 1967.

[24] N. KRYlov Lectures on Elliptic and Parabolic Equations in Holder Spaces (Graduate Studies in Mathematics, V. 12.

[25] R. Pinsky, Positive harmonic functions and diffusion. Cambridge Studies in Advanced Mathematics, 45. Cambridge University Press, Cambridge, 1995.

[26] C. Robinson- Dynamical Systems, CRC press 1995.

[27] M. ReEd, B. Simon-Methods of modern mathematical Physics, Academic Press, 1987.

[28] L. Rogers, D. Williams-Diffusions, Markov processes and Martingales, Wiley, 1987.

[29] Z. Schuss, Theory and Applications of Stochastic Differential Equations, Wiley Series in Probability and Statistics. John Wiley Sons, Inc., New York, 1980.

[30] B. Simon- Semiclassical analysis of low lying eigenvalues I. Non-degenerate Minima: Asymptotic expansion, Ann. Inst. Henri Poincaré, Vol XXXVIII, 3, (1983), 295-307.

[31] B. Simon- Semiclassical analysis of low lying eigenvalues. II tunneling. Ann. of math 120, (1984), 89-118. 
[32] B. Simon- Semi-classical analysis of low lying eigenvalues, IV. The flea on the elephant, J. Funct. Anal. 63 (1985), 123-136.

[33] S. Smale-Differential Dynamical Systems, Bull. of A.M.S, 1967 p747-830.

[34] D. Stroock-An Introduction to the Theory of Large Deviations, Springer, 1984. 